\title{
The Expanding Role of Pyridine and Dihydropyridine Scaffolds in Drug Design
}

\author{
Yong Ling' \\ Zhi-You $\mathrm{HaO}^{2}$ \\ Dong Liang ${ }^{3}$ \\ Chun-Lei Zhang ${ }^{4}$ \\ Yan-Fei Liu ${ }^{5}$ \\ Yan Wang $\mathbb{1}^{6,7}$
}

'Department of Pharmacy, The Affiliated Hospital of Qingdao University, Qingdao, Shandong, People's Republic of China;

${ }^{2}$ School of Pharmacy, Henan University of Chinese Medicine, Zhengzhou, Henan, People's Republic of China; ${ }^{3}$ State Key Laboratory for Chemistry and Molecular Engineering of Medicinal Resources, School of Chemistry and Pharmaceutical Sciences, Guangxi Normal University, Guilin, Guangxi, People's Republic of China; ${ }^{4}$ State Key Laboratory of Natural Medicines and Jiangsu Provincial Key Laboratory for TCM Evaluation and Translational Development, School of Traditional Chinese Pharmacy, China Pharmaceutical University, Nanjing, Jiangsu, People's Republic of China; ${ }^{5}$ State Key Laboratory of Bioactive Substance and Function of Natural Medicines, Institute of Materia Medica, Chinese Academy of Medical Sciences and Peking Union Medical College, Beijing, People's Republic of China; ${ }^{6} \mathrm{HEJ}$ Research Institute of Chemistry, International Center for Chemical and Biological Sciences, University of Karachi, Karachi, Pakistan; ${ }^{7}$ Institute of Food Science and Technology, Chinese Academy of Agricultural Sciences, Beijing, People's Republic of China

Correspondence: Yan Wang HEJ Research Institute of Chemistry, International Center for Chemical and Biological Sciences, University of Karachi, Karachi 75270, Pakistan

Tel +92 2I I I I-222-292 ext 164

Fax +92 21 34819018-9

Email yan.wang@iccs.edu

\begin{abstract}
Pyridine-based ring systems are one of the most extensively used heterocycles in the field of drug design, primarily due to their profound effect on pharmacological activity, which has led to the discovery of numerous broad-spectrum therapeutic agents. In the US FDA database, there are 95 approved pharmaceuticals that stem from pyridine or dihydropyridine, including isoniazid and ethionamide (tuberculosis), delavirdine (HIV/AIDS), abiraterone acetate (prostate cancer), tacrine (Alzheimer's), ciclopirox (ringworm and athlete's foot), crizotinib (cancer), nifedipine (Raynaud's syndrome and premature birth), piroxicam (NSAID for arthritis), nilvadipine (hypertension), roflumilast (COPD), pyridostigmine (myasthenia gravis), and many more. Their remarkable therapeutic applications have encouraged researchers to prepare a larger number of biologically active compounds decorated with pyridine or dihydropyridine, expandeing the scope of finding a cure for other ailments. It is thus anticipated that myriad new pharmaceuticals containing the two heterocycles will be available in the forthcoming decade. This review examines the prospects of highly potent bioactive molecules to emphasize the advantages of using pyridine and dihydropyridine in drug design. We cover the most recent developments from 2010 to date, highlighting the ever-expanding role of both scaffolds in the field of medicinal chemistry and drug development.
\end{abstract}

Keywords: nitrogen heterocycles, pharmaceuticals, bioactive compounds, current trend, substituent effect

\section{Introduction}

Heterocycles are intricately woven into basic processes of life and play a crucial role in the pharmaceutical and agrochemical industries. ${ }^{1}$ In terms of pharmacological, physicochemical, pharmacokinetic, and toxicological properties, heterocyclic structures are found in $>90 \%$ of newly synthesized and marketed drugs. ${ }^{2}$ Medicinal chemistry has evolved from an empirical practice involving the synthesis of novel substances and then gauging their biological activity. ${ }^{3}$ A plethora of synthetic compounds with heterocyclic structural frameworks have been identified - with privileged six-membered $\mathrm{N}$-containing pyridine and dihydropyridine rings linked to a diverse range of bioactivity. ${ }^{4-7}$ In the realm of six-membered heterocyclic structures, they have unique and interesting characteristics. Owing to their therapeutic potential, medicinal chemists have recently become drawn toward scaffolds in order to synthesize a wide range of novel bioactive molecules. ${ }^{8}$

In pharmaceutical targets, pyridine and its precursor molecule dihydropyridine are among the most prevalent structural units. ${ }^{9} 10$ In plants, they are mostly found in the alkaloids. ${ }^{11}$ In biological systems, redox reactions of nicotinamide adenine dinucleotide 
(NAD) reduces its pyridine ring into dihydropyridine, rendering NADH. Similar redox reactions are also present in anabolic reactions involving NAD phosphate $\left(\mathrm{NADP}^{+} / \mathrm{NADPH}\right)$ interconversion. $^{12} \mathrm{~A}$ glance at the US Food and Drug Administration (FDA) database reveals that pyridine- and dihydropyridine-containing drugs constitute nearly $14 \%$ and $4 \%$ of $N$-heterocyclic drugs approved by the agency (Figure 1). For these $18 \%$ of drugs, the major therapeutic areas of focus are infectious diseases, inflammation, the nervous system, and oncology.

Substitution-type analysis of pyridine-containing drugs revealed that the ring is mostly monosubstituted $(60 \%)$ in the database, whereas di-, tri-, and tetrasubstitution represented $22 \%, 12 \%$, and $6 \%$, respectively (Figure 2A). For dihydropyridine-containing drugs, neither mono- nor disubstitution was observed for the $N$-heterocyclic ring. However, trisubstitution of the dihydropyridine ring was the most abundant substitution type for this class of drugs. Tetra-, penta-, and hexasubstitution on the $N$-heterocyclic ring were $11 \%, 21 \%$, and $5 \%$, respectively (Figure 2B).

In recent years, synthetic chemists have been focusing on developing new analogues that employ pyridine or dihydropyridine templates in their molecular design, in order to study their mechanisms of action to discover new pharmaceutical leads. The importance of the two heterocycles in medicinal chemistry and chemical sciences can be seen by the sheer number of publications appearing between 2010 and 2020 (Figure 3).

Most reviews on this topic have concerned synthetic strategies to prepare pyridine- or dihydropyridine-containing compounds. $^{2,10,13-16}$ For either of the two scaffolds, one can also find many reviews delineating their therapeutic potential concerning a specific malady. ${ }^{17-28}$ Most reviews on pyridinecontaining compounds have examined only their anticancer potential. $^{21,22}$ Likewise, several reviews on dihydropyridinecontaining compounds typically scrutinized their calcium

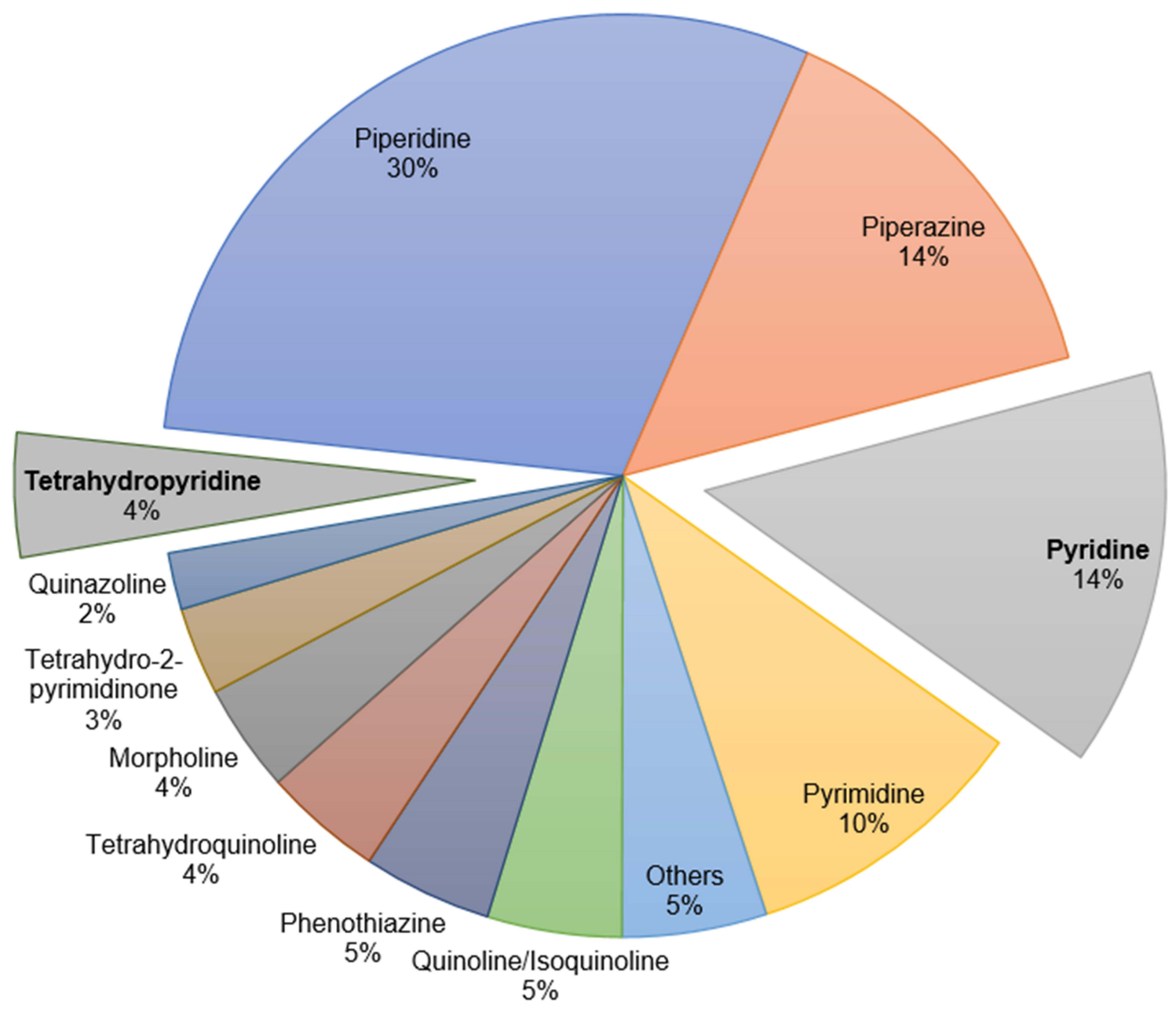

Figure I Distribution of $\mathrm{N}$-heterocyclic drugs in the FDA database. 


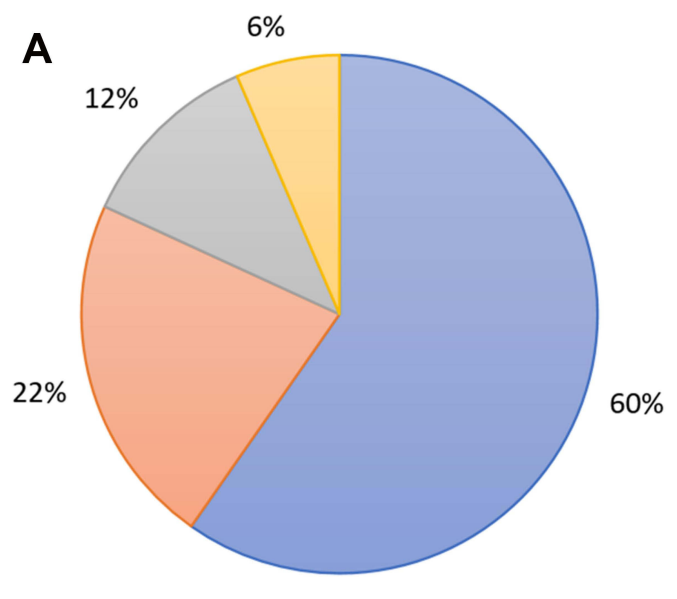

$\square$ Mono- $\square$ Di- $\square$ Tri- $\square$ Tetra-
B

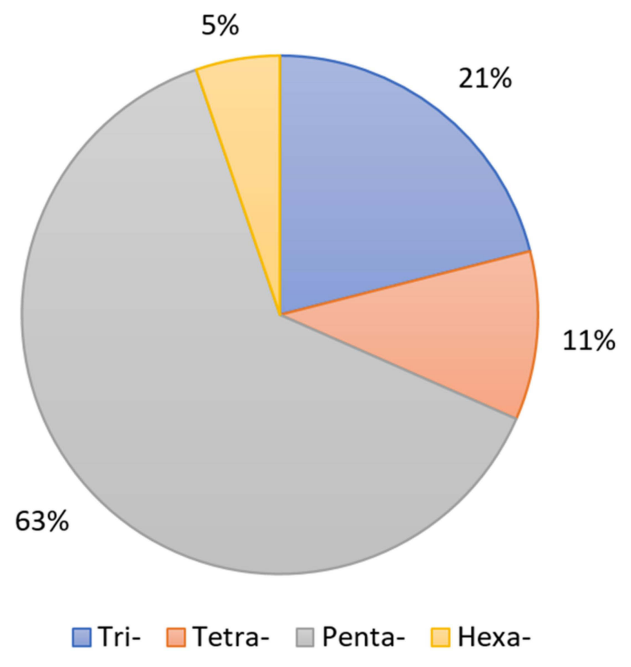

Figure 2 Substitution-type analysis of pyridine- (A) and dihydropyridine (B)-containing FDA-approved drugs.

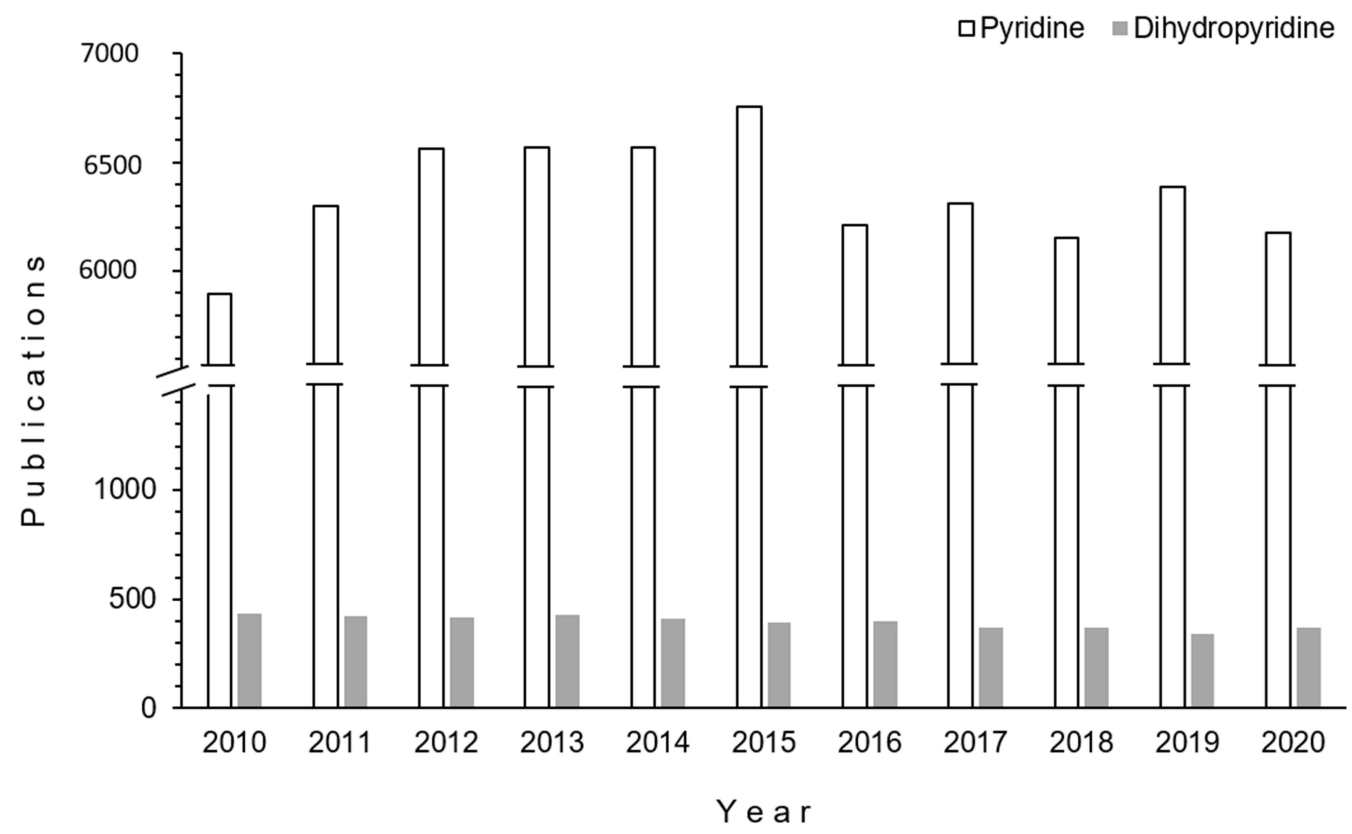

Figure 3 Publications on pyridine- and dihydropyridine-containing compounds, 2010-2020 (source: Scopus and SciFinder).

channel-blocking abilities for the treatment of hypertension and associated ailments. ${ }^{19,24,26-28}$ The neuroprotective ability of dihydropyridine derivatives has also been examined. ${ }^{23}$ Lapidot et al summarized the antibacterial activity of dihydropyridine-containing peptidomimetics. ${ }^{25}$ For substituted $1,4-$ dihydropyridine, medicinal versatility and anticipable therapeutic effects have been the focus of past reviews, ${ }^{29-31} \mathrm{eg}$, Khedkar et al briefly discussed the pharmacological importance of this type of molecule. ${ }^{30}$ To the best of our knowledge, a review focusing on the therapeutic potential of pyridine- and dihydropyridine-containing compounds has never been published. Herein, we present commercially available drugs while discussing the major therapeutic potential of synthetic bioactive molecules with either of the two scaffolds. The review covers a sizable period in the scientific literature, including the publications from 2010 to date, thereby providing a broad picture of approved drugs and bioactivity reported for pyridineor dihydropyridine-containing compounds, and is valuable material for those interested in exploring this class of compounds for further medicinal and clinical applications. 


\section{Pyridine and Dihydropyridine Scaffolds in Natural Products and Commercially Available Drugs}

Pyridine and dihydropyridine generate a suite of versatility when it comes to creating libraries of compounds with different functional groups for screening against different biological targets. Many natural products contain pyridinebased rings (Figure 4), including the vitamins (niacin and vitamin $\mathrm{B}_{6}$ ), coenzymes (NAD, NADP), alkaloids (trigonelline, $[-]$-oxirene, $[+]$-anabasine, huperzine $\mathrm{A}$, paecilomide, cystine), antibiotics (nikkomycin, collismycin), and many more compounds. ${ }^{11}$

In pharmaceuticals, nitrogen-containing heterocycles are considered instrumental structural constituents. ${ }^{32}$ The presence of pyridine or dihydropyridine ring systems can have a substantial impact on pharmacological profiles of drugs and bioactive molecules. ${ }^{33}$ For example, a pyridine motif in a drug improves its biochemical potency and metabolic stability, enhances permeability, and fixes protein-binding issues. ${ }^{33}$ Some interesting examples of the pyridine effect are highlighted in Figure 5: Vanotti et al were able to develop the potent Cdc7 kinase inhibitor 2 by substituting the phenyl group of $\mathbf{1}$ with pyridine. ${ }^{34}$ Similarly, metabolic stability of thiourea-based nicotinamide phosphoribosyltransferase inhibitor 3 is improved 160-fold when its terminal phenyl ring is replaced with pyridine in $4{ }^{35} \mathrm{~A}$ heterocyclic pyridine ring in a molecule is also capable of enhancing its cellular permeability. For example, Doller et al identified a pyridine-containing positive allosteric modulator 6 with 190 -fold the cellular permeability of $5{ }^{36}$ For the treatment of schizophrenia, proteinbinding issues of positive allosteric modulator 7 were resolved by the introduction of an additional pyridine ring in $8 .^{37}$ It is thus pertinent to say that substitution of nitrogen-containing heterocyclic rings profoundly affects the physicochemical properties of the bioactive molecule. ${ }^{33}$

There is a plethora of commercially available drugs in the market which contain pyridine rings, such as abiraterone for prostate cancer, ${ }^{38}$ enpiroline for malaria, ${ }^{39}$ nicotinamide for pellagra ${ }^{40}$ nikethamide as a respiratory stimulant, ${ }^{41}$ piroxicam for arthritis, ${ }^{42}$ isoniazid for tuberculosis, ${ }^{43}$ pyridostigmine for myasthenia gravis, ${ }^{44}$ tropicamide as an antimuscarinic, ${ }^{45}$ doxylamine for allergies, ${ }^{46}$ omeprazole for ulcers, ${ }^{47}$ delavirdine as an antiviral against HIV/ AIDS ${ }^{48}$ enisamium iodide for influenza, ${ }^{49}$ and tacrine as an inhibitor of the AChE enzyme ${ }^{50}$ for Alzheimer's disease prevention (Figure 6).
Dihydropyridine ring-containing drugs mostly act as calcium-channel blockers, ${ }^{51}$ and are frequently employed for the treatment of hypertension and heart-related problems. ${ }^{52}$ Such drugs include nilvadipine, nifedipine, amlodipine, azelnidipine, clevidipine, felodipine, and pranidipine. Some of these drugs are also used to cure many other therapeutic conditions. ${ }^{53}$ For example, nifedipine is being used for Raynaud's syndrome and premature birth. ${ }^{54}$ Dihydropyridine-containing huperzine, a natural product, acts as an AChE inhibitor and is employed in the treatment of Alzheimer's disease, whereas ciclopirox is widely used as an antifungal agent to cure ringworm and athlete's foot disease (Figure 7).

Milrinone and amrinone are the two commercially available vasodilators, ${ }^{55}$ and contain both pyridine and dihydropyridine ring systems in their structures (Figure 8). In general, pyridine- and dihydropyridine-containing drugs are mostly used as antimicrobial, antiviral, anticancer, antioxidant, antihypertensive, antidiabetic, antimalarial, and antiinflammatory agents, psychopharmacological antagonists, and antiamebic agents. ${ }^{56-62}$ A comprehensive list of commercially available drugs containing pyridine and/or dihydropyridine scaffolds and their mechanism of action is summarized in Table 1.

Analysis of the substitution pattern in FDA-approved drugs has revealed that the 1,4-dihydropyridine ring in the drugs was mostly substituted at the para-position (4). Disubstitution at both ortho-positions (2 and 6) were observed in eleven drugs, while one drug had monosubstitution at the ortho-position (2). Similarly, three drugs had monosubstitution at the meta-position (3), whereas ten drugs had disubstitution at meta-positions ( 3 and 5) of the 1,4-dihydropyridine ring. For brevity, the substitution patterns of pyridine and dihydropyridine ring systems in FDAapproved drugs are illustrated in Figure 9.

\section{Pharmacological Activity}

On the therapeutic front, pyridine- and dihydropyridine-containing compounds possess versatile bioactivity, due to which they are the integral in numerous drugs. The literature revealed many examples wherein this class of compounds demonstrated promising pharmacological properties.

\section{Cardiovascular Drugs and Bioactive Compounds}

Hypertension is among the main risk factors of cardiovascular disease. Many different types of antihypertensive 
<smiles>C[n+]1cccc(C(=O)[O-])c1</smiles>

Trigonelline<smiles>C[C@@H]1CCc2c(CO)cncc21</smiles>

(-)-Tecostidine

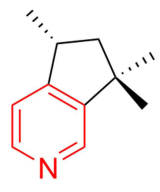

(-)-Oxirine<smiles>c1cncc([C@@H]2CCCCN2)c1</smiles>

(+)-Anabasine<smiles>C[C@@H]1CCc2c(C(=O)O)cncc21</smiles>

(-)-Plantagonine<smiles>Cc1cncc2c1CC[C@H]2C</smiles>

(-)-Actinidine<smiles>O=C(O)c1cccnc1</smiles>

Niacin<smiles>CN1CCCC1c1cccnc1</smiles>

Nicotine<smiles>Cc1ncc(CO)c(CO)c1O</smiles>

Pyridoxine<smiles>C[C@@H]1CCc2c(C=O)cncc21</smiles>

(-)-Indicaine<smiles>C[C@H]([C@H](N)C(=O)N[C@H](C(=O)O)[C@H]1O[C@@H](n2ccc(=O)[nH]c2=O)[C@H](O)[C@@H]1O)[C@@H](O)c1ccc(O)cn1</smiles>

Nikkomycin Z<smiles>COC1=C(N)C(=O)c2nc(-c3nc(C(=O)O)c(C)c(-c4cc(OC)c(OC)cc4O)c3N)ccc2C1=O</smiles><smiles>COc1cc(-c2ccccn2)nc(/C=N/O)c1SC</smiles><smiles>NC(=O)C1=CN([C@@H]2O[C@H](COP(=O)(O)OP(=O)(O)OC[C@H]3O[C@@H](n4cnc5c(N)ncnc54)[C@H](O)[C@@H]3O)[C@@H](O)[C@H]2O)C=CC1</smiles>

NADH Co-enzyme

$\mathrm{Cl} N$

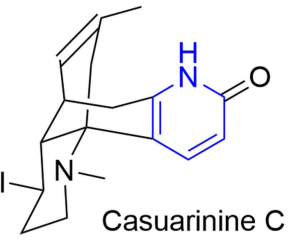<smiles>[R15][R15]([H])([H])[H]</smiles><smiles>O=c1c(O)ccc2n1C[C@H]1CNC[C@H]2C1</smiles>

$(1 R, 5 S)$

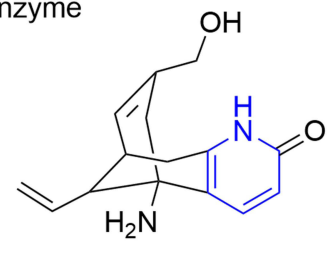

Lycoparine A<smiles></smiles>

Huperzine A<smiles>CC1(C)OC(=O)c2c1cc[nH]c2=O</smiles>

Cerpegin<smiles>COc1cc([C@@H](Cc2ccccc2)n2cc(C(N)=O)c(=O)cc2Cc2ccccc2)oc(=O)c1</smiles><smiles>O=C(O)[C@H]1CCCC[C@H]1c1c[nH]c(=O)cc1O</smiles>

Paecilomide<smiles>O=C(O)C[C@@H]1[C@@H](C(=O)O)NC[C@H]1c1ccc(C(=O)O)[nH]c1=O</smiles>

Acromelic acid A<smiles>NC(Cc1cc(C(=O)O)[nH]c(=O)c1)C(=O)O</smiles>

Acromelobic acid

Figure 4 Pyridine and dihydropyridine ring system in medicinally important natural products.

drugs are employed to treat the problem. Main classes of such drugs include $\alpha$ - and $\beta$-adrenergic inhibitors, renin inhibitors, vasodilators, diuretics, calcium-channel blockers, angiotensin converting-enzyme inhibitors, and many more (Figure 10). Pyridine-containing torsemide is an
FDA-approved drug that promotes diuresis, thereby lowering the blood pressure of the patient. Pyridine- and dihydropyridine-containing amrinone and milrinone are beta-adrenergic blockers, also called $\beta$-blockers, to help manage hypertension via vasodilation and ultimately save 


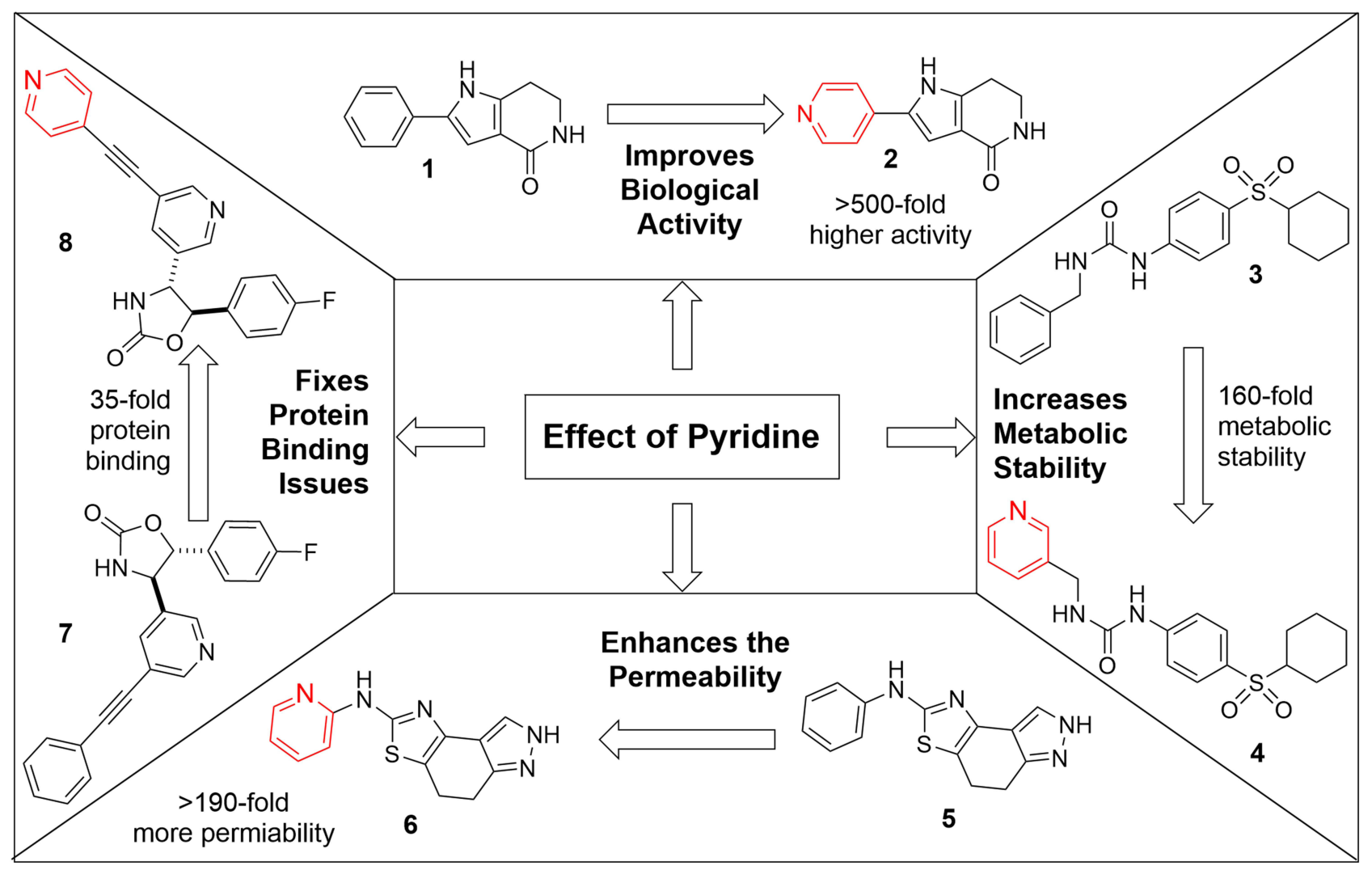

Figure 5 Effect of pyridine on key pharmacological parameters.

the patient from a second heart attack. Disruption of calcium movement through cellular channels is another strategy to lower blood pressure. Most of the calcium antagonists in the FDA database contain dihydropyridine scaffolds, with penta-substitution pattern observed for the ring (Figure 11).

In 2014, N-propargyl-substituted derivatives of 1,4DHP derivatives were synthesized by Rucins et al, who incorporated pharmacophore moieties into their structures and investigated their calcium channel-blocking activity. In SH-SY5Y-type neuroblastoma cells (which contain $\mathrm{Ca}^{2+}$ channels of L-and N-type) and A7r5 cells (which are rat aortic muscle cells expressing L-type $\mathrm{Ca}^{2+}$ channels), and impact of newly synthesized compounds on intracellular concentration of calcium $\left[\mathrm{Ca}^{2+}\right]$ was studied. Among the series, compounds $\mathbf{9}$ and $\mathbf{1 0}$ with $n$-dodecyl pyridinium moiety as an amphiphilic group exhibited the strongest calcium antagonistic activity in SH-SY5Y neuroblastoma cells $\left(\mathrm{IC}_{50}=5-14 \mathrm{mM}\right)$, and $\mathrm{A} 7 \mathrm{r} 5$ type lines $\left(\mathrm{IC}_{50}=0.6-0.7\right.$ $\mathrm{mM}$ ). These compounds demonstrated moderately effective antioxidant activity. Compound $\mathbf{1 0}$ had no effect on mitochondrial function at dosages comparable to that used to block L-type calcium channels, and no damage was seen in vivo. As a consequence, this compound can be regarded as safe up to $100 \mathrm{mg} / \mathrm{kg}$, with no toxicity. It was observed that the 1,4-DHP ring-bearing propargyl group at position $1 \mathrm{did}$ not significantly influence the bioactivity of the tested derivatives. Therefore, compounds with $n$-dodecyl pyridinium moiety at the para-position (Figure 11) might be the lead molecules for subsequent modifications and in vivo studies of cardiovascular and neurological disorders. ${ }^{176}$

Nitrendipine $\mathbf{1 1}$ is a DHP-type calcium antagonist with a simple structure, but low potency. Antihypertensive actions of nitrendipine analogues can be improved by increasing the alkyl-chain length at the 3 or 5 position. ${ }^{177,178}$ Zhou et al synthesized nitrendipine analogues, and their antihypertensive properties were assessed in spontaneously hypertensive rats by means of intravenous immunoglobulin administration. $S$ - and $R$-enantiomers had different calcium antagonistic activity in various studies. ${ }^{179}$ It was found that the $S$-enantiomer had 100-fold the antihypertensive properties of the $R$-enantiomer (Figure 12). Moreover, the efficacy of nitrendipine analogues was further enhanced by elongating carbon chain-length. These findings suggest that alkyl-chain length at position 5 is closely linked to the antihypertensive effects of nitrendipine analogues. DHP's antihypertensive effects 
<smiles>CN(C)C(=O)Oc1ccc[n+](C)c1</smiles>

Pyridostigmine (myasthenia gravis)<smiles>NNC(=O)c1ccncc1</smiles>

Isoniazid (tuberculosis)<smiles>CCN(CC)C(=O)c1cccnc1</smiles>

Nikethamide (respiratory stimulant)<smiles>NC(=O)c1cccnc1</smiles>

Nicotinamide (pellagra)<smiles>CN1C(C(=O)Nc2ccccn2)=C(O)c2ccccc2S1(=O)=O</smiles>

Piroxicam (arthritis)<smiles>O=C1CN=C(c2ccccn2)c2cc(Br)ccc2N1</smiles>

Bromazepam (anxiety)<smiles>Nc1c2c(nc3ccccc13)CCCC2</smiles>

Tacrine (Alzheimer's disease)<smiles>COc1ccc2[nH]c(S(=O)Cc3ncc(C)c(OC)c3C)nc2c1</smiles>

Omeprazole (ulcer)<smiles>Nc1ccc(N=Nc2ccccc2)c(N)n1</smiles>

Phenazopyridine (urinary tract analgesic)

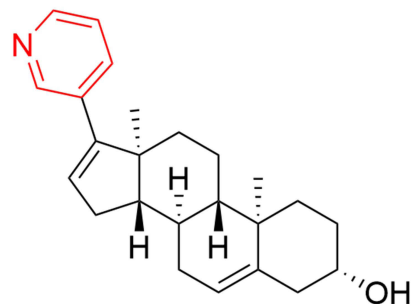

Abiraterone (prostate cancer)<smiles>CC(C)Nc1cccnc1N1CCN(C(=O)c2cc3cc(NS(C)(=O)=O)ccc3[nH]2)CC1</smiles>

Delavirdine (HIVIAIDS)<smiles>O=C(NCCO[N+](=O)[O-])c1cccnc1</smiles>

Nicorandil (vasodilator)<smiles>CN(C)CCOC(C)(c1ccccc1)c1ccccn1</smiles>

Doxylamine (allergy)

Figure 6 Some commercially available drugs containing the pyridine scaffold.

were attenuated by extra-long or extra-short alkyl chains at position 5. On position 5 of DHP, an alkyl chain containing seven carbon atoms is the most appropriate length. Therefore, the strongest antihypertensive effect was observed for 5-n-heptyl-3-methyl-2,6-dimethyl-4-(3-nitrophenyl)-1,4dihydropyridine-3,5-dicarboxylate $[( \pm)-12]$. Antihypert ensive effects of $( \pm)-\mathbf{1 2}$ and +-12 were compared, with +-isomer being 1.79 times more potent at a dosage of $2 \mathrm{mg} / \mathrm{kg}$ than the raceme. ${ }^{180}$
Calcium channel-antagonistic activity of phenyl amino imidazolyl-bearing 1,4-dihydropyridines were also investigated by Zarghi et al. His research group replaced the ortho-nitrophenyl group at the para-position of nifedipine with a 2-methylthio-1-phenylamino-5-imidazolyl substituent. Then, calcium channel-antagonist activity $\left(\mathrm{IC}_{50}\right)$ were determined in guinea pigs, which revealed that the contractile responses elicited by these novel dihydropyridinecontaining compounds inhibited higher $\mathrm{K}^{+}$concentrations 
<smiles>CC=C1C2C=C(C)CC1(N)c1ccc(=O)[nH]c12</smiles>

Huperzine A (AChE inhibitor) Alzheimer's disease<smiles>COC(=O)C1=C(C#N)NC(C)=C(C(=O)OC(C)C)C1c1cccc([N+](=O)[O-])c1</smiles>

Nilvadipine (hypertension)<smiles>COCCOC(=O)C1=C(C)NC(C)=C(C(=O)OC(C)C)C1c1cccc([N+](=O)[O-])c1</smiles>

Nimodipine (vasospasm)<smiles>CC1=CCN(O)C(C2CCCCC2)=C1</smiles>

Ciclopirox (ringworm \& athlete's foot)<smiles>CC1=C(C(=O)OC(C)C)C(c2cccc([N+](=O)[O-])c2)C(C(=O)OC2CN(C(c3ccccc3)c3ccccc3)C2)=C(N)N1</smiles>

Azelnidipine (hypertension)<smiles>COC(=O)C1=C(C)NC(C)=C(C(=O)OC)C1c1ccccc1[N+](=O)[O-]</smiles>

Nifedipine (Raynaud's syndrom, and premature birth)<smiles>CC1=C(C(=O)OCCN(Cc2ccccc2)c2ccccc2)C(c2cccc([N+](=O)[O-])c2)C(P2(=O)OCC(C)(C)CO2)=C(C)N1</smiles>

Efonidipine (hypertension and angina)

Figure 7 Some commercially available drugs containing the dihydropyridine scaffold.<smiles>Cc1[nH]c(=O)c(C#N)cc1-c1ccncc1</smiles><smiles>Nc1cc(-c2ccncc2)c[nH]c1=O</smiles>

Amrinone (phosphodiesterase 3 inhibitor)

Figure 8 FDA-approved vasodilators containing both pyridine and dihydropyridine scaffolds.

in a dose-dependent manner. In the muscular membrane, these effects were comparable to those shown by nifedipine and might be attributed to the suppression of $\mathrm{Ca}^{2+}$ entry via voltage-dependent calcium channels. However, it should be noted that the gut features a complex network of tissue, and we cannot rule out the potential of these compounds acting differently on distinct muscular and neuronal sites. A comparison of the activity of alkyl ester series in these compounds shows that by increasing the chain length of methylene at $\mathrm{C}_{3}$ and $\mathrm{C}_{5}$ ester substituents, activity reduces. For example, the $t$-butyl ester-containing compound was the least active among the series. Overall results suggested that most of the compounds had activity comparable to those of nifedipine, with the exception of two compounds, 13 and 14, being more active than nifedipine (Figure 13). As such, they can be potential leads for the design of calcium-channel blockers. ${ }^{181}$

Kumar et al reported anticoagulant activity for dihydropyridine-containing compounds, which was assessed using activated partial thromboplastin time and prothrombin time coagulation assays. Compound 15 (Figure 14) had a coagulation time of 720.35 seconds at $30 \mathrm{mg} / \mathrm{mL}$. The standard drug heparin was used at $\mathrm{s}$ similar concentration. ${ }^{182}$ 


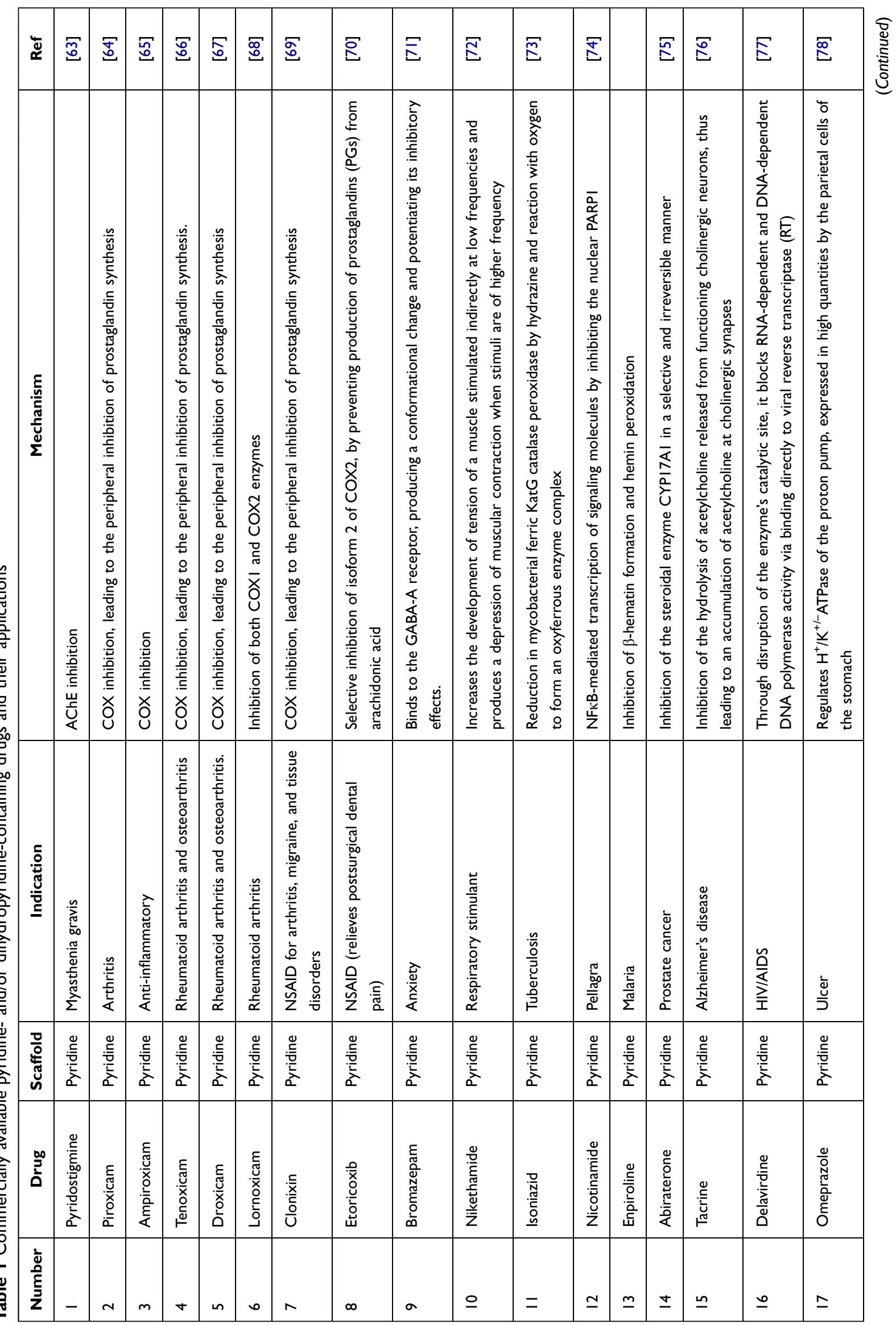




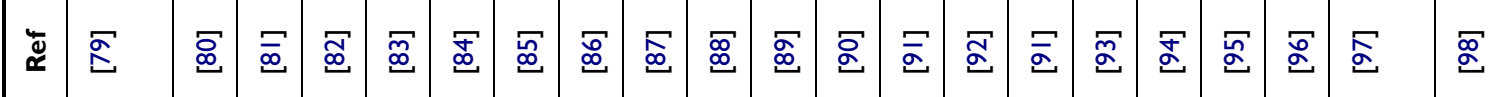

\begin{tabular}{|c|c|c|c|c|c|c|c|c|c|c|c|c|c|c|c|c|c|c|c|c|c|}
\hline 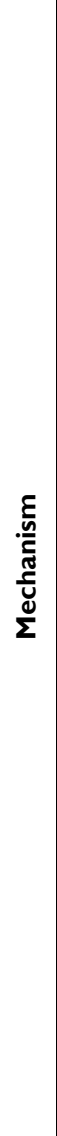 & 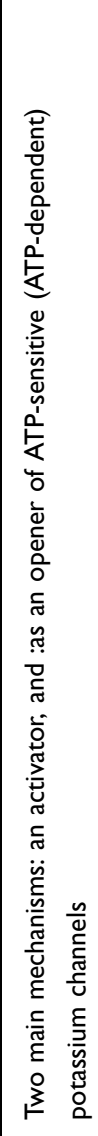 & 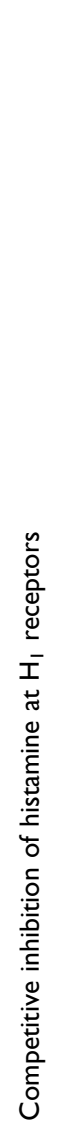 & 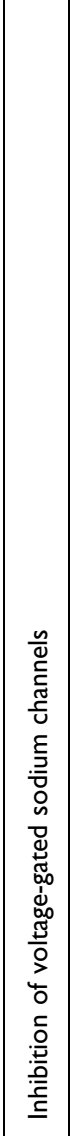 & 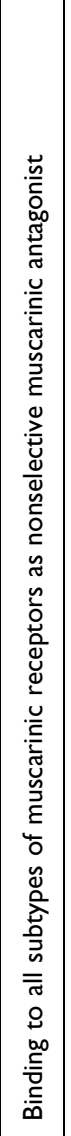 & 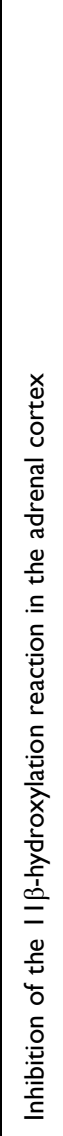 & 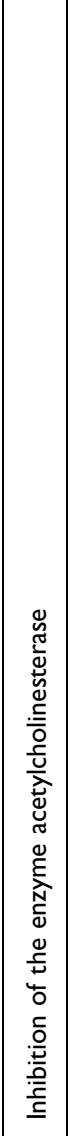 & 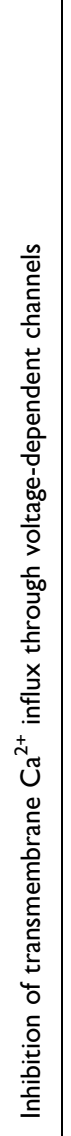 & 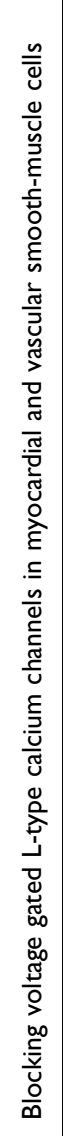 & 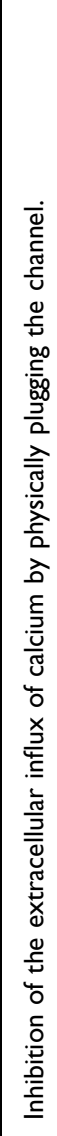 & 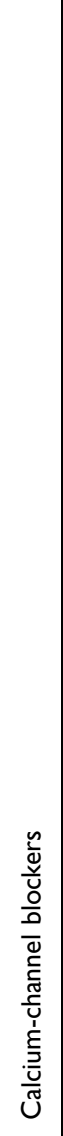 & 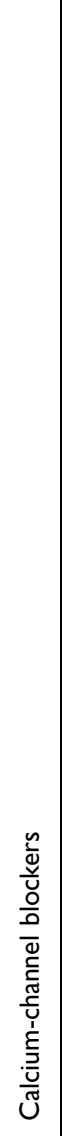 & 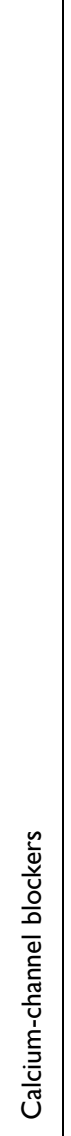 & 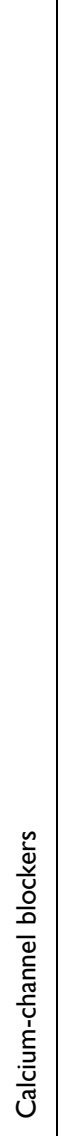 & 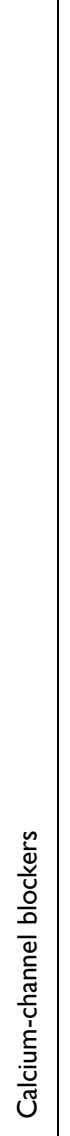 & 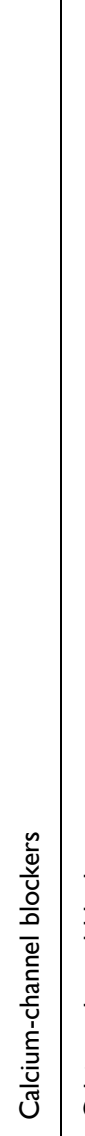 & 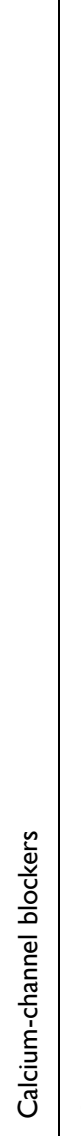 & 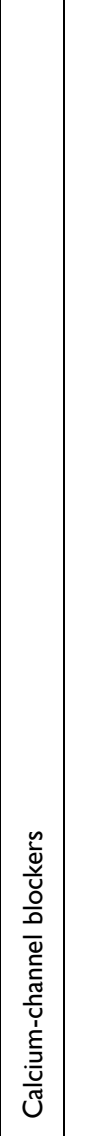 & 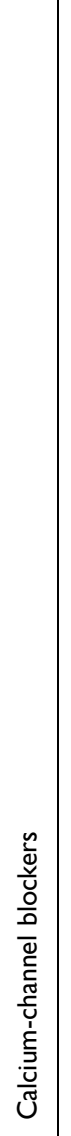 & 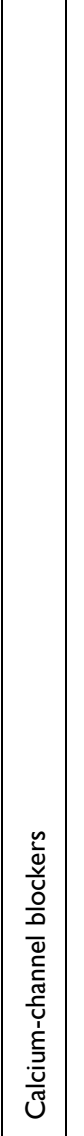 & 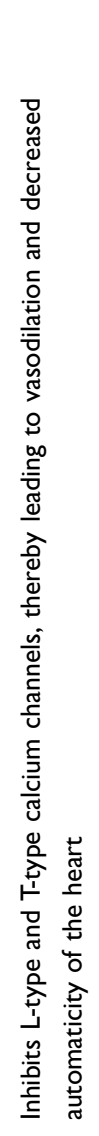 & 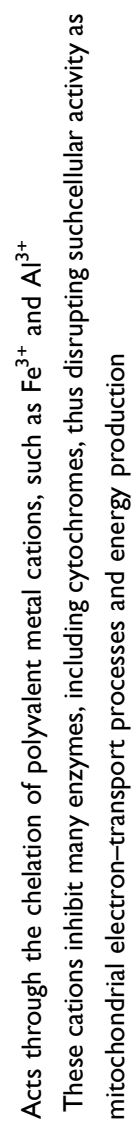 \\
\hline 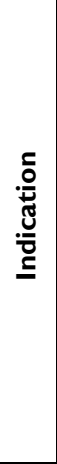 & 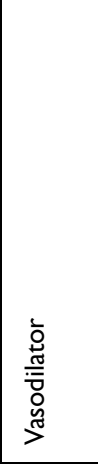 & $\frac{\vec{b}}{\stackrel{\vec{b}}{\bar{\psi}}}$ & 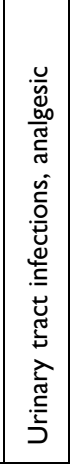 & 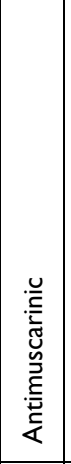 & 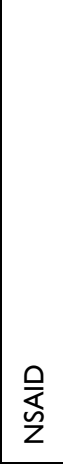 & 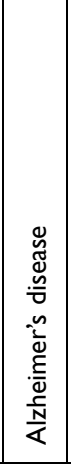 & 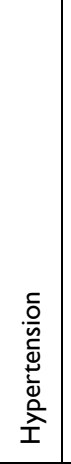 & 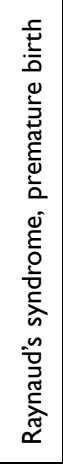 & 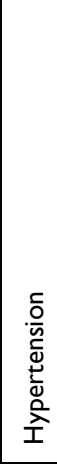 & 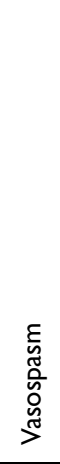 & 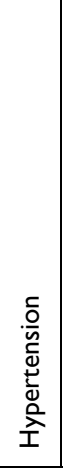 & 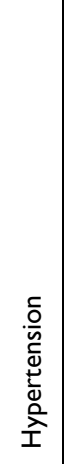 & 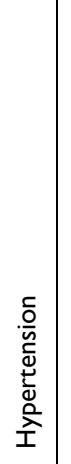 & 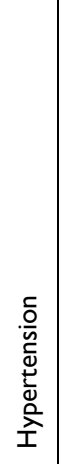 & 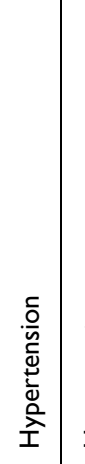 & 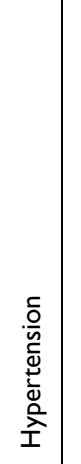 & 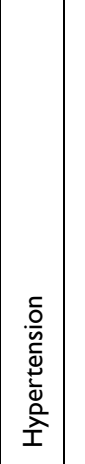 & 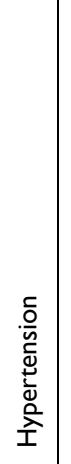 & 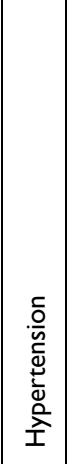 & 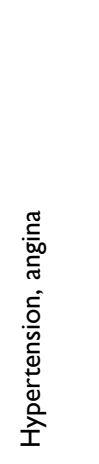 & 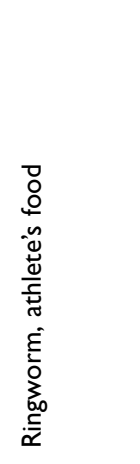 \\
\hline $\begin{array}{l}\frac{\pi}{0} \\
\stackrel{0}{0} \\
\text { n } \\
\end{array}$ & 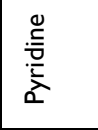 & 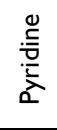 & 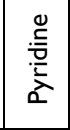 & 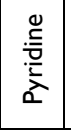 & 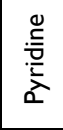 & $\begin{array}{l}\stackrel{a}{د} \\
\underline{\Delta}\end{array}$ & $\frac{\hat{O}}{\square}$ & 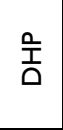 & $\begin{array}{l}\stackrel{a}{1} \\
0\end{array}$ & $\begin{array}{l}\frac{a}{\mathbf{T}} \\
\frac{1}{2}\end{array}$ & $\stackrel{\underline{\mathbf{T}}}{\mathbf{D}}$ & $\begin{array}{l}\stackrel{\hat{T}}{\mathrm{D}} \\
\mathrm{a}\end{array}$ & 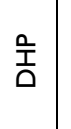 & $\stackrel{\underline{\mathbf{T}}}{\mathrm{I}}$ & $\frac{\stackrel{a}{1}}{\Delta}$ & $\frac{\grave{1}}{\underline{\Delta}}$ & 竞 & $\stackrel{\underline{\mathbf{T}}}{\mathrm{I}}$ & $\begin{array}{l}\frac{a}{T} \\
\Delta\end{array}$ & $\stackrel{\stackrel{a}{\mathbf{T}}}{\underline{\Delta}}$ & $\begin{array}{l}\frac{\hat{T}}{\mathrm{D}} \\
\mathrm{a}\end{array}$ \\
\hline 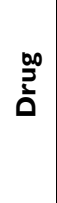 & \begin{tabular}{|c}
$\overline{\bar{z}}$ \\
$\overline{\bar{c}}$ \\
$\overline{\bar{\nu}}$ \\
$\stackrel{\bar{\nu}}{\bar{z}}$
\end{tabular} & 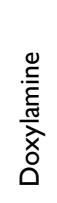 & 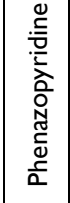 & 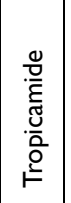 & 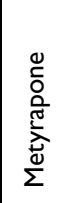 & 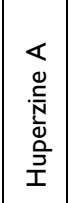 & 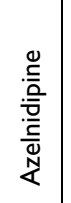 & 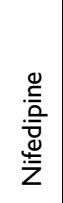 & 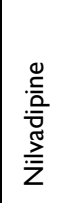 & 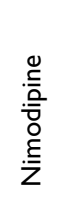 & 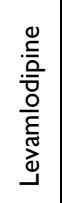 & 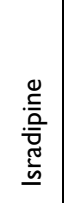 & 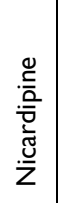 & 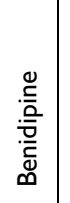 & 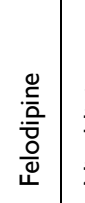 & 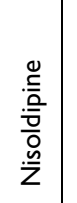 & 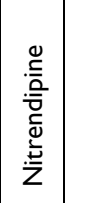 & 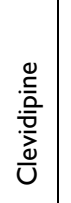 & 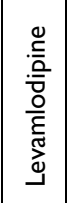 & 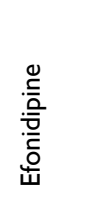 & 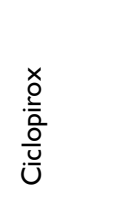 \\
\hline $\begin{array}{l}\text { ऐे } \\
\text { है } \\
\overline{\mathbf{z}}\end{array}$ & $\underline{\infty}$ & $\underline{a}$ & กิ & $\bar{N}$ & 4 & $\tilde{N}$ & $\stackrel{d}{\sim}$ & $\vec{N}$ & $\sim$ & $\lambda$ & $\widetilde{N}$ & సे & p & $\bar{m}$ & $\tilde{m}$ & 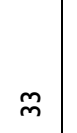 & mे & $\stackrel{m}{m}$ & m & $\hat{m}$ & $\infty_{m}$ \\
\hline
\end{tabular}




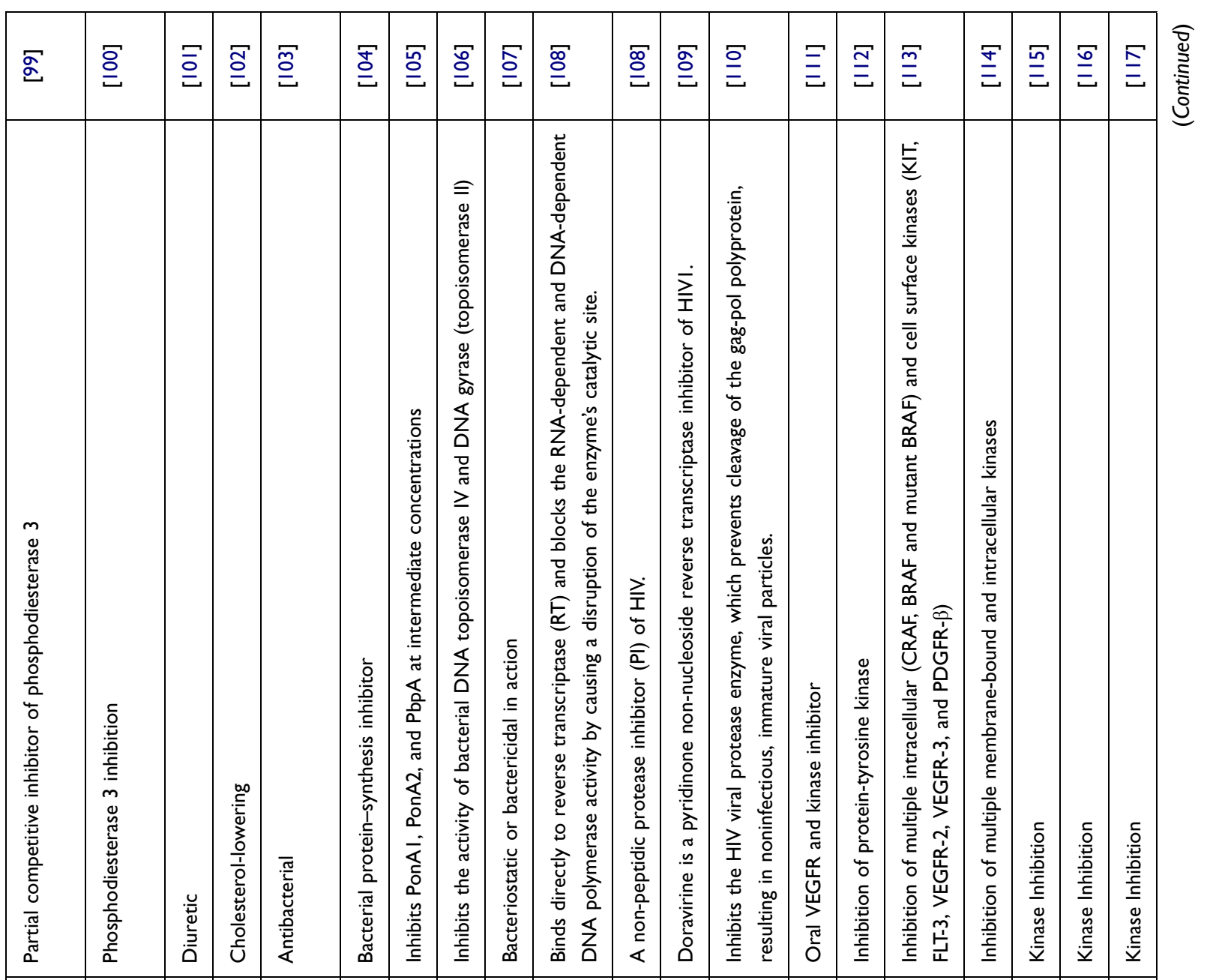

\begin{tabular}{|c|c|c|c|c|c|c|c|c|c|c|c|c|c|c|}
\hline & $\mid$ & 旁言 & & 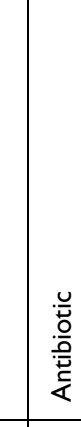 & & 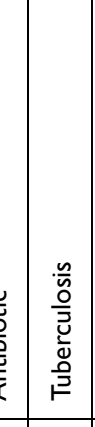 & $\overline{\mathbf{I}}$ & & 蔓 & 屁 & & & & \\
\hline 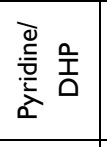 & 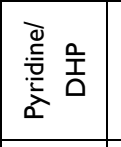 & 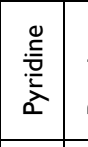 & 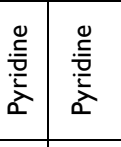 & 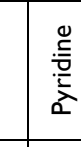 & & $\frac{0}{\frac{2}{2}}$ & $\stackrel{\underline{x}}{\underline{x}}$ & 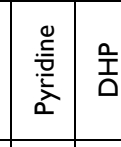 & 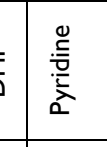 & 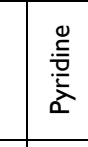 & & & & \\
\hline 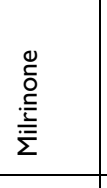 & \begin{tabular}{|l} 
高 \\
毫
\end{tabular} & 点 & 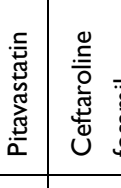 & 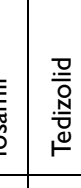 & & 童 & & 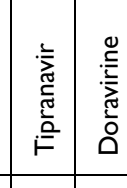 & 亨 & \begin{tabular}{l}
$\frac{0}{3}$ \\
\multirow{3}{*}{+}
\end{tabular} & & & & 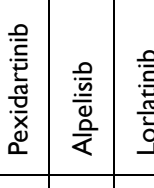 \\
\hline & 8 & $\bar{F}$ & & $\ddagger$ & & & & q & $\bar{n}$ & $\approx$ & & & & $\stackrel{n}{n}$ \\
\hline
\end{tabular}




\begin{tabular}{|c|c|c|c|c|c|c|c|c|c|c|c|c|c|c|c|c|c|c|}
\hline 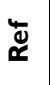 & $\stackrel{\underline{\infty}}{\underline{\Xi}}$ & $\stackrel{\sigma}{\Xi}$ & & ¿্் & $\overline{\bar{\Xi}}$ & $\underset{\mathbb{Z}}{\mathbb{Z}}$ & 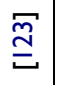 & $\underset{\Xi}{\stackrel{\Xi}{\Xi}}$ & $\stackrel{\Xi}{\Xi}$ & $\overline{\overline{0}}$ & 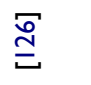 & 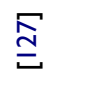 & $\stackrel{\text { D্ }}{\stackrel{D}{U}}$ & হ్ & $\stackrel{\bar{p}}{\underline{\varrho}}$ & $\overline{\bar{m}}$ & $\stackrel{\widetilde{\widetilde{N}}}{\underline{\Xi}}$ & $\stackrel{m}{\stackrel{m}{U}}$ \\
\hline 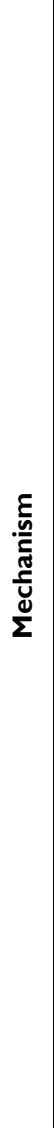 & 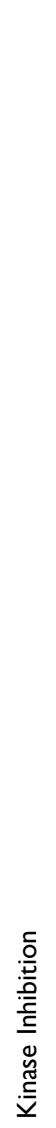 & 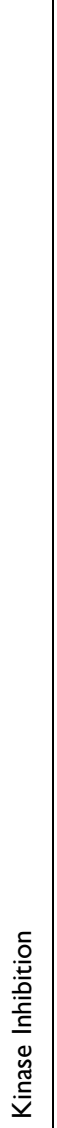 & 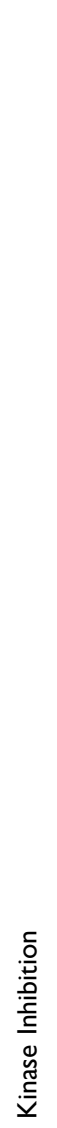 & 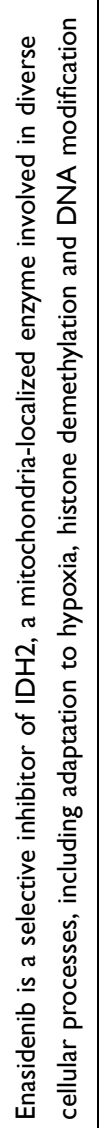 & 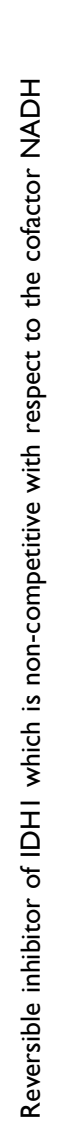 & 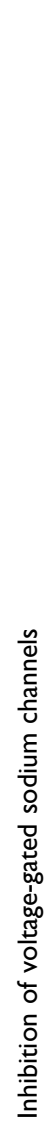 & 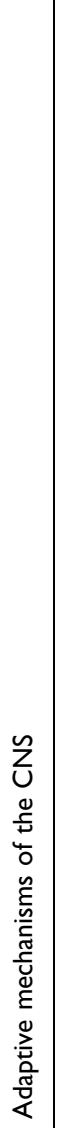 & 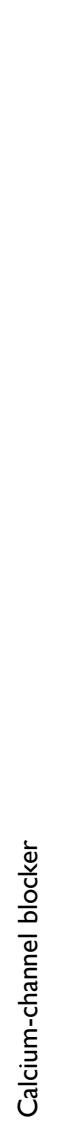 & 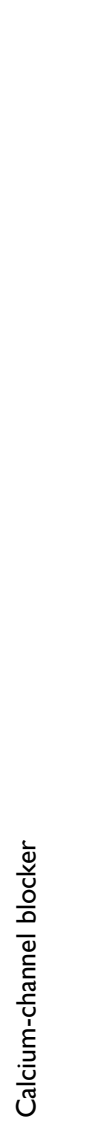 & 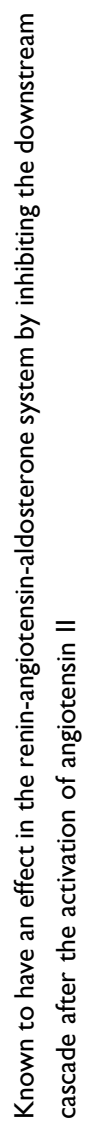 & 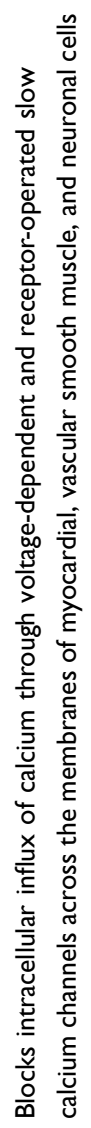 & 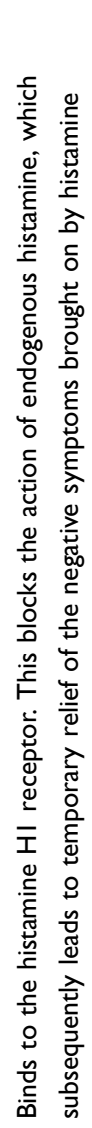 & 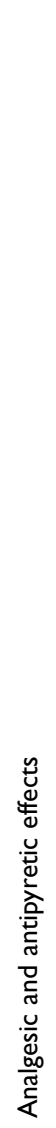 & 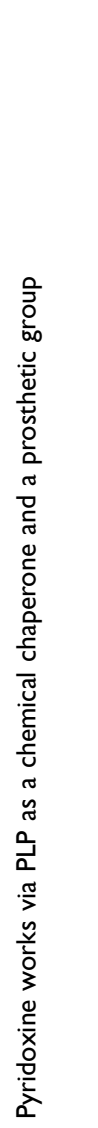 & 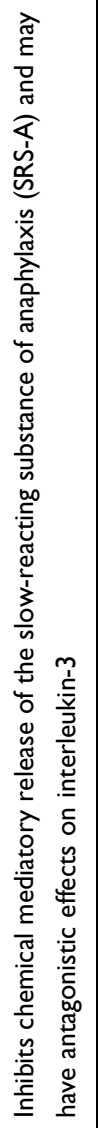 & 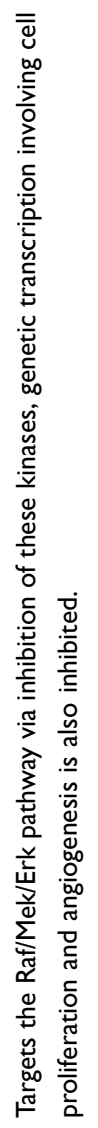 & 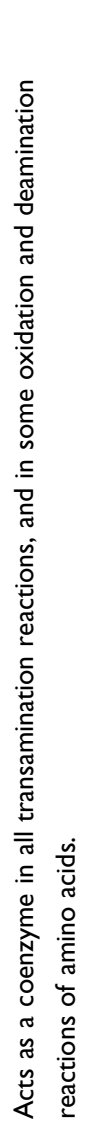 & 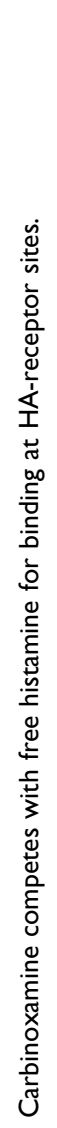 \\
\hline 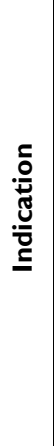 & 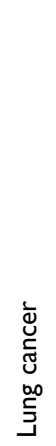 & 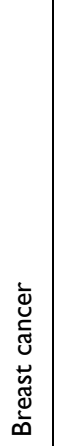 & 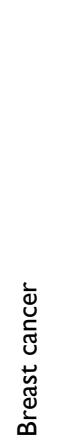 & 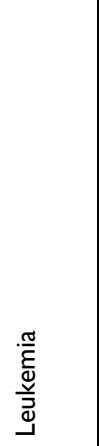 & 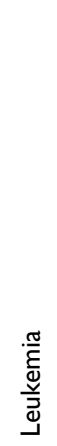 & 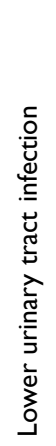 & 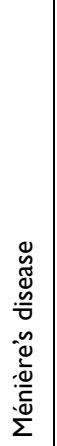 & 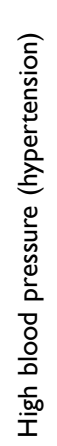 & 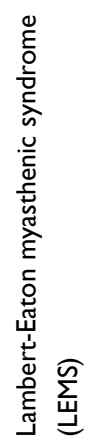 & 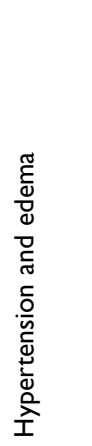 & 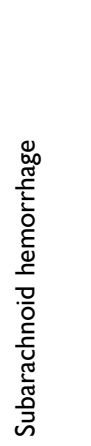 & 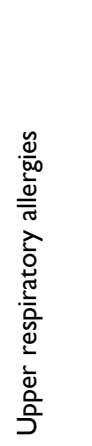 & 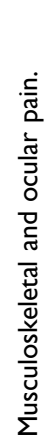 & 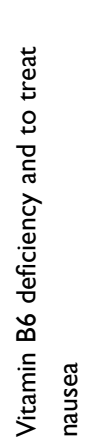 & 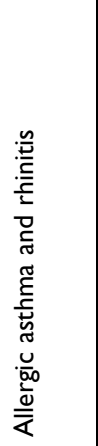 & 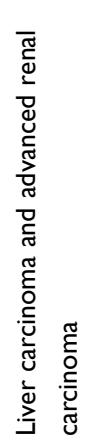 & 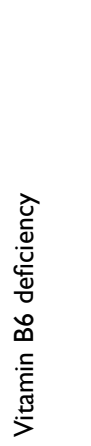 & 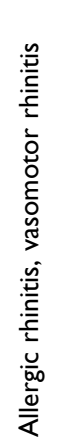 \\
\hline 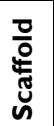 & 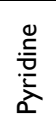 & 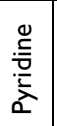 & 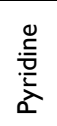 & 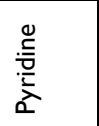 & 竞 & 总 & 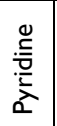 & 竞 & 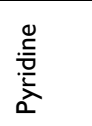 & 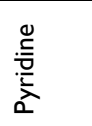 & 옴 & 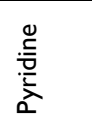 & 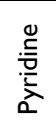 & 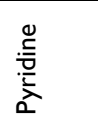 & 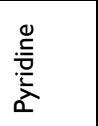 & 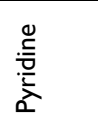 & 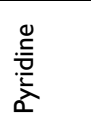 & 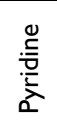 \\
\hline 足 & 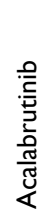 & 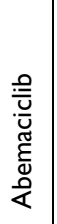 & 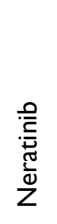 & 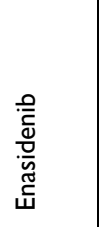 & 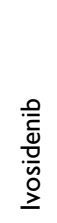 & 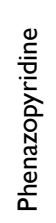 & 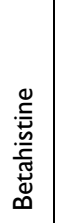 & $\begin{array}{l}\stackrel{0}{\circ} \\
\stackrel{\overline{0}}{\overline{0}} \\
\frac{0}{0} \\
\end{array}$ & 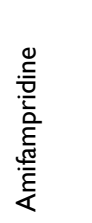 & 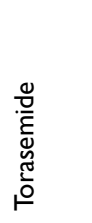 & 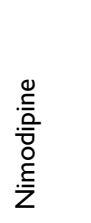 & 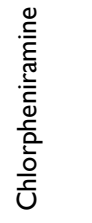 & 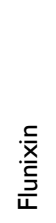 & 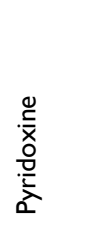 & 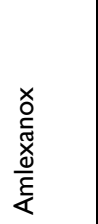 & 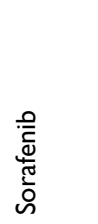 & 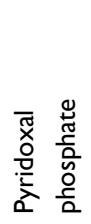 & 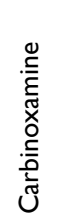 \\
\hline$=$ & ตे & 8 & $\overline{0}$ & $\widetilde{\sigma}$ & $\tilde{\boldsymbol{b}}$ & t & น & 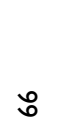 & $\hat{6}$ & ఖి & 。 & R & $\pi$ & N & $\kappa$ & \pm & $\stackrel{n}{n}$ & r \\
\hline
\end{tabular}




\begin{tabular}{|c|c|c|c|c|c|c|c|c|c|c|c|c|c|c|c|}
\hline 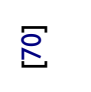 & 苞 & 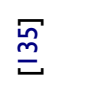 & 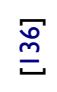 & 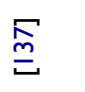 & 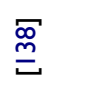 & $\stackrel{\sigma}{\underline{\nu}}$ & 定 & $\overline{\bar{\Xi}}$ & $\stackrel{\widetilde{F}}{\Xi}$ & $\stackrel{\bar{\sigma}}{\underline{v}}$ & \multicolumn{2}{|l|}{ 守 } & $\stackrel{\sqrt[p]{5}}{\underline{v}}$ & 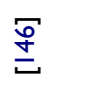 & $\underset{\text { 㝍 }}{\underline{5}}$ \\
\hline 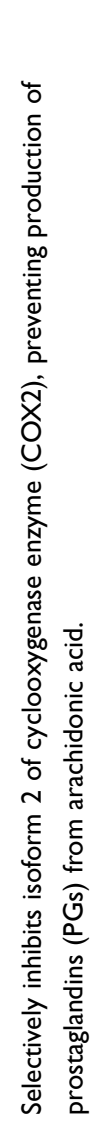 & & 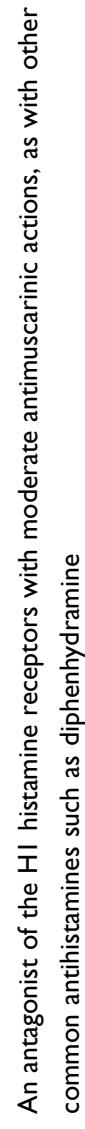 & 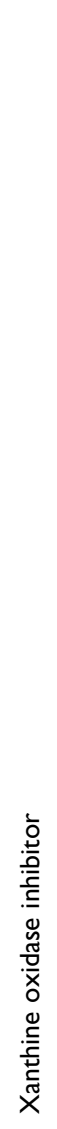 & 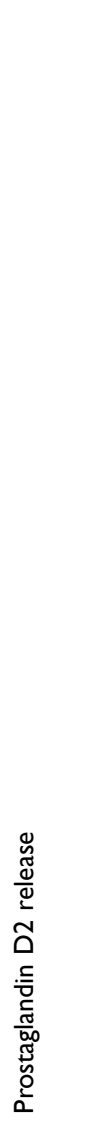 & 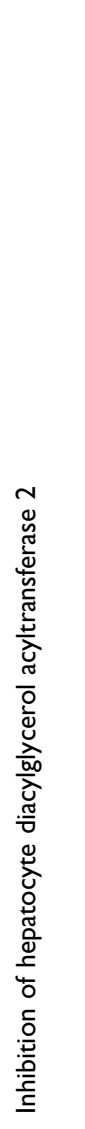 & 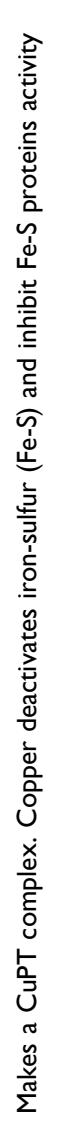 & 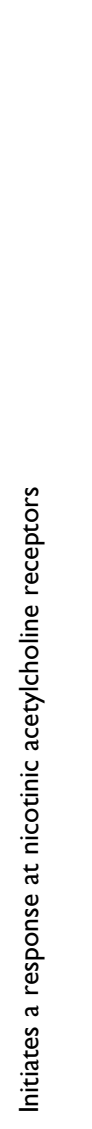 & 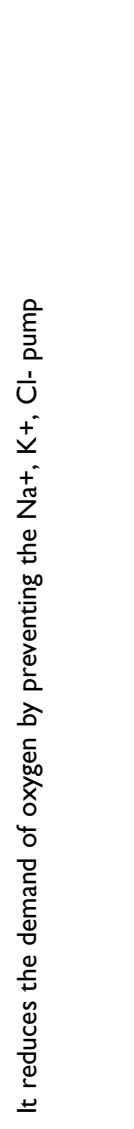 & 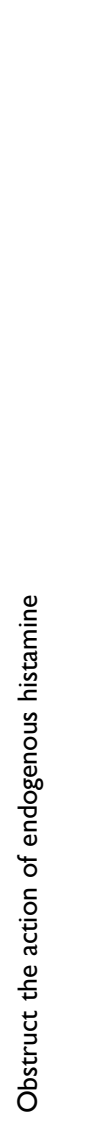 & 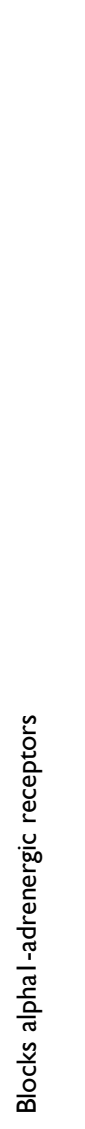 & 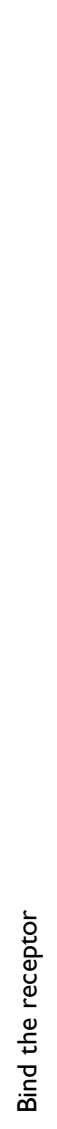 & 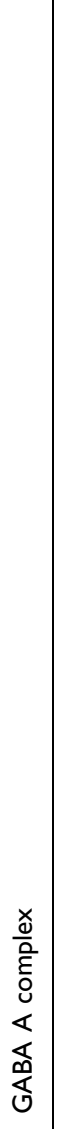 & 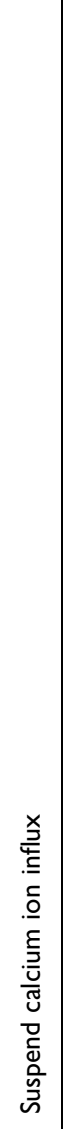 & 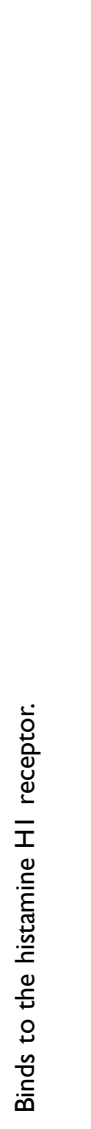 & 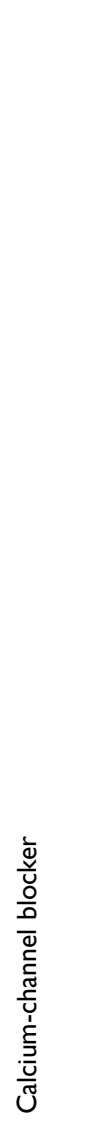 \\
\hline 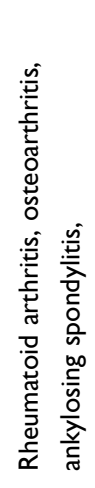 & $\begin{array}{l}\frac{\mathscr{\omega}}{00} \\
\bar{\omega} \\
\frac{\tilde{\omega}}{\bar{\sigma}}\end{array}$ & 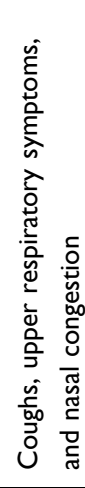 & 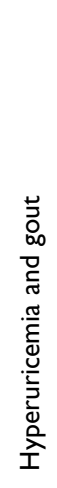 & 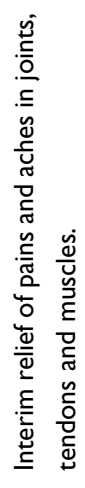 & 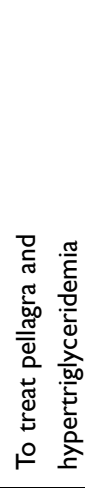 & 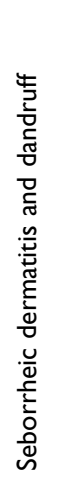 & 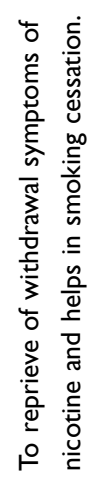 & 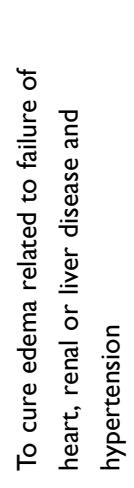 & 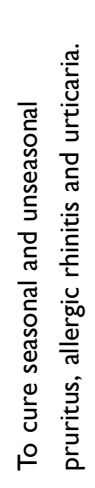 & 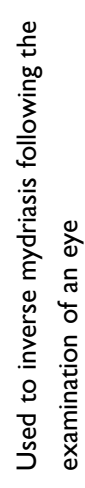 & 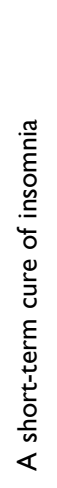 & & 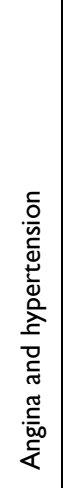 & 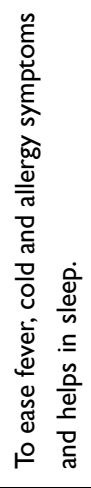 & 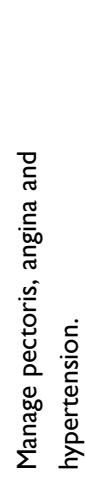 \\
\hline 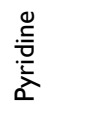 & 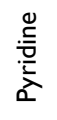 & 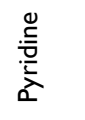 & 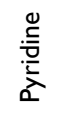 & 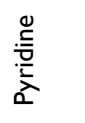 & 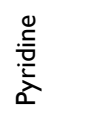 & 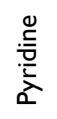 & 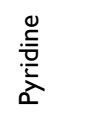 & 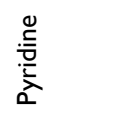 & 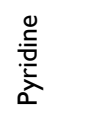 & & 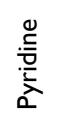 & & $\begin{array}{l}\stackrel{a}{\mathbf{I}} \\
\underline{\Delta}\end{array}$ & 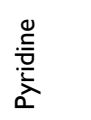 & $\frac{0}{\mathbf{T}}$ \\
\hline 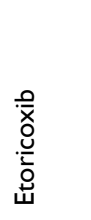 & 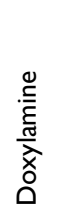 & 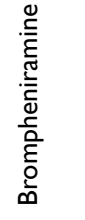 & 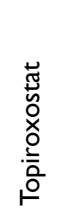 & 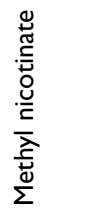 & 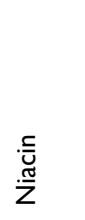 & 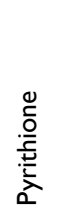 & 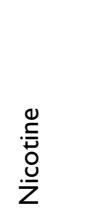 & 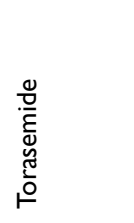 & 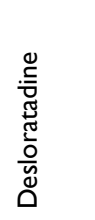 & 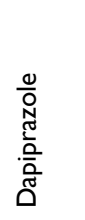 & $\begin{array}{l}\frac{\varepsilon}{0} \\
\frac{0}{0} \\
\frac{0}{0} \\
N\end{array}$ & & 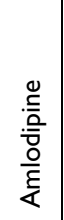 & 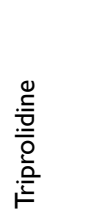 & 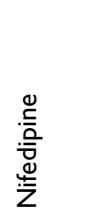 \\
\hline$\hat{A}$ & $\stackrel{\infty}{\curvearrowright}$ & হ & $\infty$ & $\bar{\infty}$ & ఓ & $\infty$ & Ф & 岕 & œ & $\widehat{\infty}$ & $\infty$ & & ळ & \& & $\bar{a}$ \\
\hline
\end{tabular}




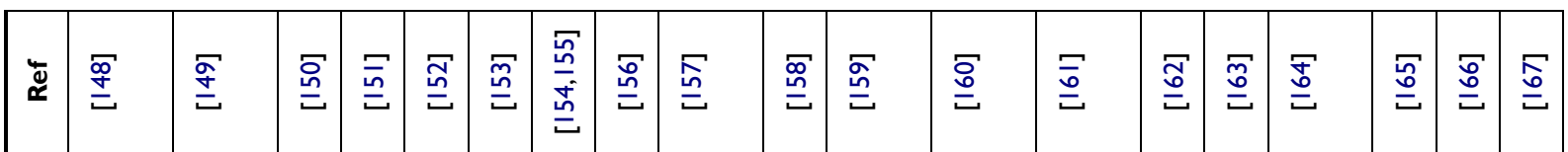

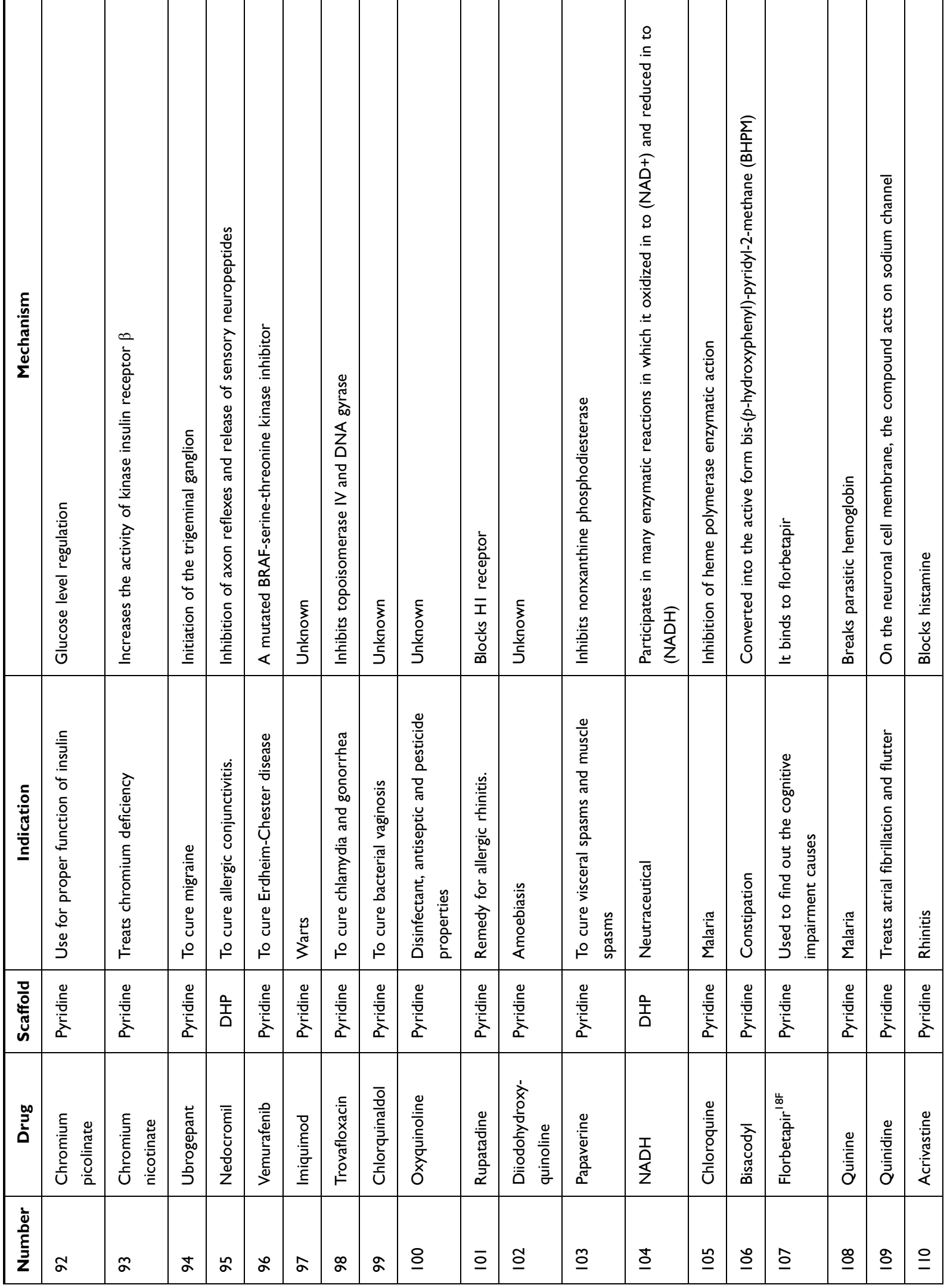




\begin{tabular}{|c|c|c|c|c|c|c|c|c|c|}
\hline 总 & 完 & $\stackrel{\Gamma}{\varrho}$ & 믈 & $\bar{\Xi}$ & 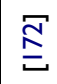 & $\stackrel{\underline{N}}{\underline{\Xi}}$ & $\bar{\Sigma}$ & $\stackrel{\text { 守 }}{\Xi}$ & $\stackrel{\Gamma}{\underline{\Omega}}$ \\
\hline 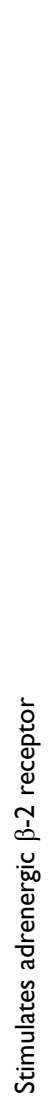 & 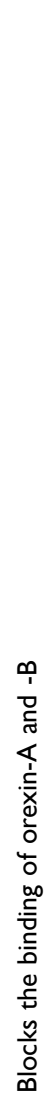 & 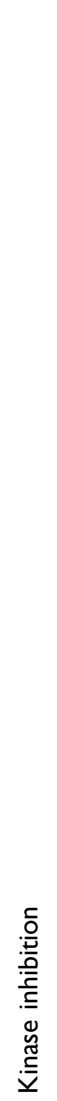 & 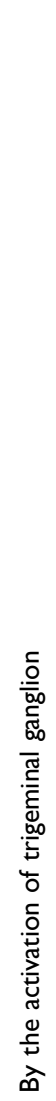 & 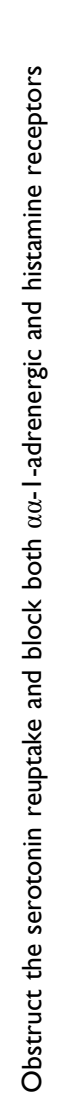 & 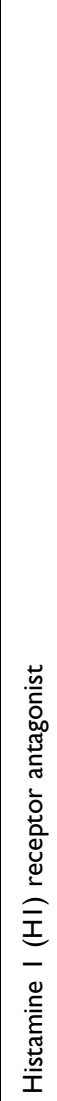 & 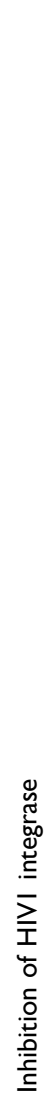 & 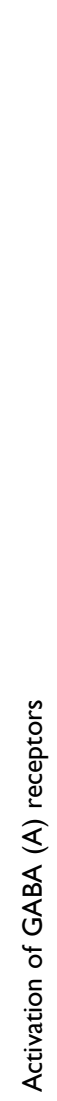 & 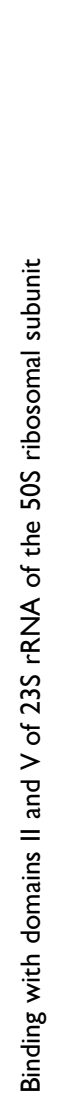 & 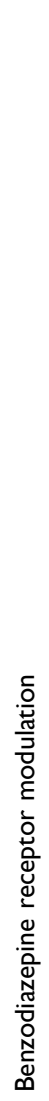 \\
\hline 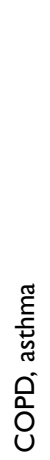 & 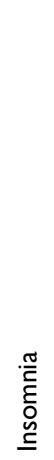 & 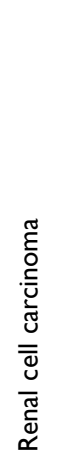 & 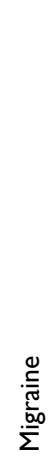 & 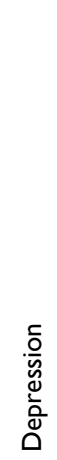 & 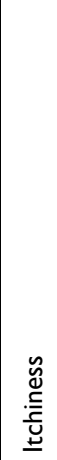 & 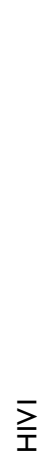 & 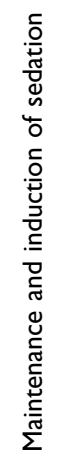 & 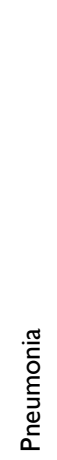 & $\begin{array}{l}\text { 号 } \\
\underline{\underline{\underline{\varepsilon}}} \\
\underline{\underline{\underline{g}}}\end{array}$ \\
\hline 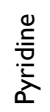 & 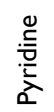 & 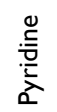 & 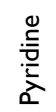 & 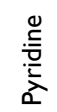 & 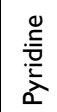 & 呈 & 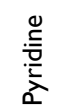 & 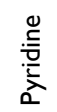 & 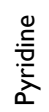 \\
\hline $\begin{array}{l}\overline{0} \\
\overline{0} \\
\tilde{J} \\
\underline{\tilde{J}} \\
\underline{\underline{0}}\end{array}$ & 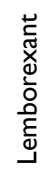 & 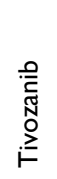 & 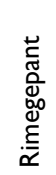 & $\begin{array}{l}0 \\
\stackrel{0}{0} \\
\stackrel{0}{0} \\
\stackrel{N}{ٌ}\end{array}$ & 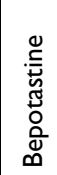 & 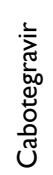 & 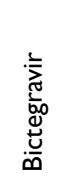 & 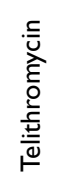 & $\begin{array}{l}\frac{0}{0} \\
\frac{0}{\mathrm{~L}} \\
\overline{\mathrm{O}}\end{array}$ \\
\hline$\equiv$ & $\underline{\beth}$ & $\stackrel{m}{\underline{\underline{n}}}$ & $\stackrel{ \pm}{=}$ & $\stackrel{\underline{n}}{=}$ & ㅡㅡ & 气 & $\stackrel{\infty}{=}$ & $\stackrel{\varrho}{\underline{\underline{O}}}$ & ิㅗ \\
\hline
\end{tabular}

Dyslipidemia is a complex disease that promotes atherosclerosis and cardiovascular problems. ${ }^{183}$ In 2001, the pyridine-containing drug cerivastatin was withdrawn from the market due to rhabdomyolysis risk. Later, another drug in the statin class, pitavastatin, was developed for lowering blood cholesterol (Figure 15).

In 2016, antihyperlipidemic compounds of type (benzoylphenyl)pyridine-3-carboxamide were reported (Figure 16), and 16-17 demonstrated excellent in vivo activity. ${ }^{184}$ The antidyslipidemic activity of the compounds were tested in vivo.

These compounds were able to lower total cholesterol (TC) from $11.0 \%$ to $24.8 \%$. The compounds showed plasma phospholipid-lowering (PL) activity ranging from $5.7 \%$ to $28.5 \%$. The most active compound in the series was 17 (-28.5\%), whereas gemfibrozil (39.1\% PLdecreasing activity) was the least active. The scavenging capability of compounds was also tested against the production of superoxide ions $\left(\mathrm{O}^{2-}\right)$ using 100 and $200 \mathrm{mg} /$ $\mathrm{mL}$ allopurinol. Antioxidant activity of the compounds in the series was substantial, and those with tert-butyl ester functionality were found to be the most active. Compounds 18-22 demonstrated potential antidyslipidemic potential, but compounds 21-22 exhibited considerable antioxidant activity (Figure 17). It can tentatively be assumed that the ester group plays a key role in differentiating antidyslipidemic and antioxidant activity. Antidyslipidemic potential was shown by those compounds that contained methyl/ethyl ester groups, whereas antioxidant activity was displayed by compounds with tert-butyl ester functionality. ${ }^{185}$

Although many dihydropyridine-containing drugs are commercially available to manage hypertension, we, believe that further research in the pyridine-dihydropyridine class of compounds will lead to the discovery of new pharmaceuticals to handle complications associated with cardiovascular diseases.

\section{Anti-Infectious Drugs and Bioactive Compounds Antibacterial Agents}

Antibiotic resistance is a serious threat to public health, driving the search for novel inhibitors of bacteria. Most antibiotics are being resisted by bacterial pathogens, rendering the development of novel, more effective antibacterial drug candidates a critical requirement. In the last 10 years, the FDA has approved many pyridine-containing 

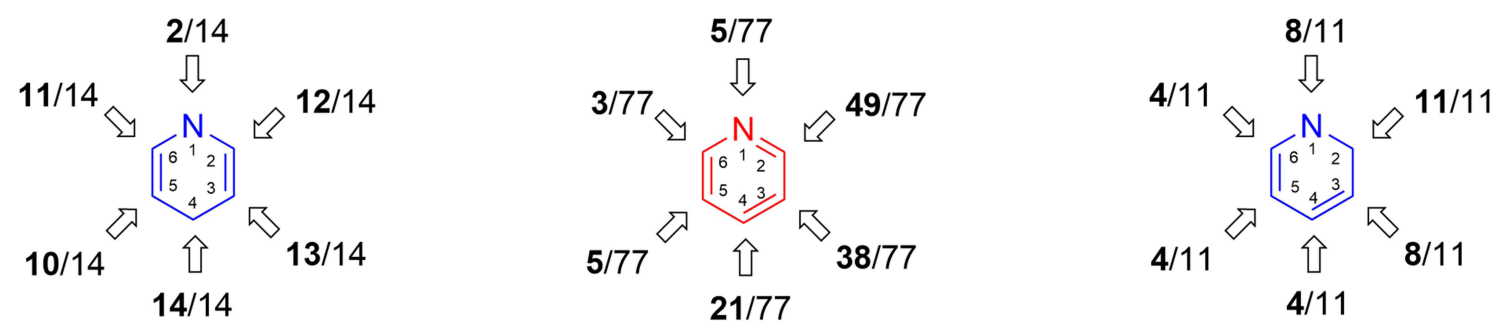

Figure 9 Substitution-pattern analysis in pyridine and dihydropyridine in FDA-approved drugs.

antibiotics, such as ceftaroline fosamil, tedizolid, ceftazidime, and delafloxacin (Figure 18).

Jo et al synthesized oxazolidinone-bearing pyridine derivatives, which had potent antibacterial activity. In vitro and in vivo antibacterial studies were performed against troublesome Gram-negative and Gram-positive strains of bacteria and two antibiotic-resistant strains. Several substituted (hetero)aromatic rings were tolerated on the pyridine moiety, and the presence or orientation of the methyl group in the (hetero)aromatic rings had a profound effect on antibacterial activity. The most active derivatives, 23-25 (Figure 19), displayed potent activity against a wide range of drug-resistant bacteria, as well as Moraxella catarrhalis and Haemophilus influenzae, and had longer half-lives in vivo than linezolid. They had 4-16 fold the in vitro activity of linezolid, and and double the in vivo efficacy. ${ }^{186}$

In another study, oxazolo[4,5-b]pyridine derivatives were synthesized to derive antimicrobial agents. These compounds had excellent activity against methicillin-resistant Staphylococcus aureus, which is responsible for widespread hospital-acquired infections. Compounds $\mathbf{2 8}$ and $\mathbf{3 1}$ were the most potent, with MIC values of 1.56-25 $\mu \mathrm{g} / \mathrm{mL}$, while 26, 27, 29, 30, 32, and 33 showed moderate activity $(6.25-50 \mu \mathrm{g} / \mathrm{mL})$ in comparison to the conventional drugs streptomycin and ampicillin (Figure 20). Further studies revealed that oxazolo[ $[4,5-b]$ pyridine analogues were more active against Gram-positive bacteria than Gram-negative bacteria. Compounds $\mathbf{2 8}$ and $\mathbf{3 1}$ had potent activity against S. aureus methicillin-resistant strains, with activity of 1.56-3.12 $\mu \mathrm{g} / \mathrm{mL}$, while standard drugs (ampicillin and streptomycin) had MIC values of $6.25-12.5 \mu \mathrm{g} / \mathrm{mL}$. These compounds were also found to be active against other bacterial strains. Furthermore, the synthesized compounds were docked in the enterotoxin protein of $S$. aureus, which is a type A staphylococcal enterotoxin. Significant antibacterial activity for $\mathbf{2 8}$ and $\mathbf{3 1}$ in comparison to the standard drugs ampicillin and streptomycin was further validated by in vitro and in silico studies. The compounds were then tested for ligand-protein binding (MRSA protein) affiniy toward S. aureus, wherein the compounds had higher ligand-protein binding affinity than the stand drugs. ${ }^{187}$

Novel pyrazolo[3,4- $b]$ pyridine derivatives of 4-thiazolidinone Schiff bases, and azetidin-2-ones have also been synthesized and screened for antimicrobial activity. ${ }^{188}$ Most compounds exhibited moderate-high activity at 0.12-62.5 $\mu \mathrm{g} / \mathrm{mL}$, wherein amphotericin $\mathrm{B}$, ampicillin, and gentamicin were used as standard antimicrobial agents. Against the Fusarium oxysporum fungal strain, compound 37 had an MIC of $0.98 \mu \mathrm{g} / \mathrm{mL}$, comparable to that of the standard antimicrobial drug amphotericin B. Significant cytotoxic activity was observed for these compounds against the HepG2 cell line, with $\mathrm{IC}_{50}$ of 0.0158 $71.3 \mu \mathrm{M}$ in comparison to doxorubicin $\left(\mathrm{IC}_{50}=0.008 \mu \mathrm{M}\right)$. Compounds 34-38 also had antiproliferative activity $\left(\mathrm{IC}_{50}\right.$ $=0.0001-0.0211 \mu \mathrm{M})$ against the MCF7 cell line (Figure 21). Specifically, highly significant antiproliferative efficacy was displayed by compound 37 against MCF7 cells and HepG2, with $\mathrm{IC}_{50}$ of $0.0001 \mu \mathrm{M}$ and $0.0158 \mu \mathrm{M}$, respectively. These results suggest that these compounds might lead toward the development of promising and novel antimicrobial and antiproliferative drug candidates. These compounds undoubtedly hold great potential in the quest to develop novel antiproliferative and antimicrobial agents. ${ }^{188}$

Dihydropyridines bearing thiazole derivatives were initially assessed with in silico molecular docking simulations to investigate their possible DNA gyrase inhibitory activity. Antibacterial activity was then assessed to validate the results of computational studies, wherein compound 39 demonstrated the highest efficacy against Aspergillus flavus and compound $\mathbf{4 0}$ had significant potency against C. albicans and A. flavus (Figure 22). 


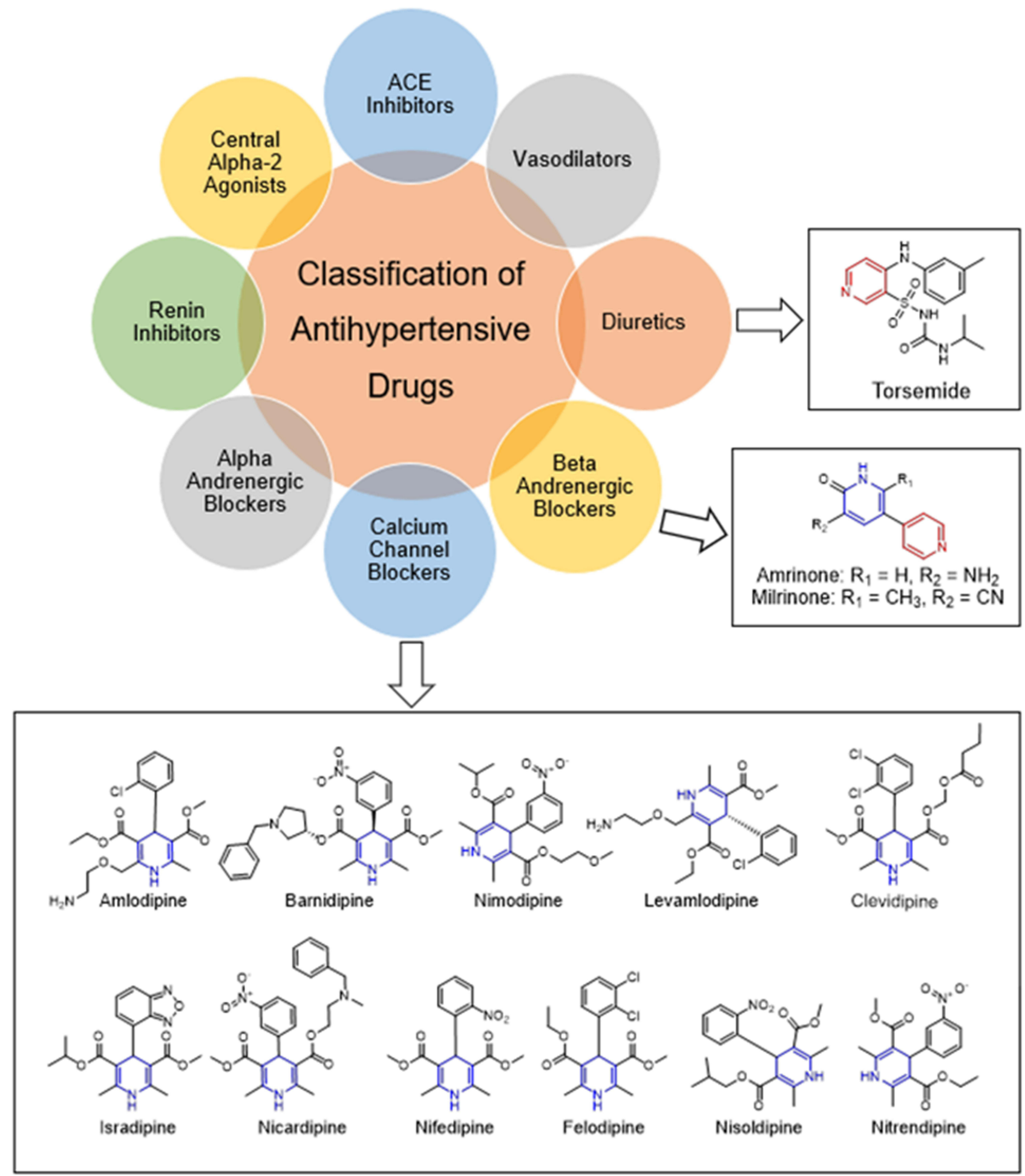

Figure 10 Dodecylpyridinium moiety containing dihydropyridines with potent calcium antagonism in the A7r5 cell line.

Because of the phenyl ring's size and inductive impact, the presence of an electron-withdrawing group might be responsible for excellent activity. ${ }^{189}$
Pyridine rings containing 1,3,4-oxadiazole derivatives were explored by Lak et al. ${ }^{190}$ All synthesized compounds were evaluated for their antibacterial activity against 
<smiles>CCOC(=O)C1=C(C)NC(C)=C(C(=O)OCC)C1c1ccc[n+](C(C)(Br)CC)c1</smiles>

9: $I C_{50}=0.7 \pm 0.3 \mathrm{mM}$<smiles>[B-][n+]1cccc(C2C(C(=O)OCC)=C(C)N(CC#C)C(C(C)(C)C(C)C)=C2C(=O)OCC)c1</smiles>

10: $I C_{50}=0.6 \pm 0.06 \mathrm{mM}$

Figure II FDA-approved drugs containing pyridine or dihydropyridine scaffolds for the treatment of hypertension.

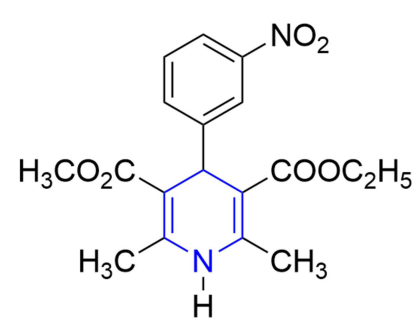

11<smiles>CCCCCCCOC(=O)C1=C(C)NC(C)=C(C(C)=O)[C@H]1c1cccc([N+](=O)[O-])c1</smiles>

$(+)-12$

Figure 12 Highly potent calcium-channel antagonists.<smiles>[R]OC(=O)C1=C(C)NC(C)=C(C([R])O[R])C1c1cnc(SC)n1Nc1ccccc1</smiles>

13: $\mathrm{R}=\mathrm{Et}$

$1.69 \pm 0.43 \times 10^{-9} \mathrm{nM}$<smiles>[R20]C(=O)C1=C(C)NC(C)=C(C([R])[R])C1c1cnc([Si])n1Nc1ccccc1</smiles>

14: $R=$ Benzyl

$1.49 \pm 0.39 \times 10^{-9} \mathrm{nM}$

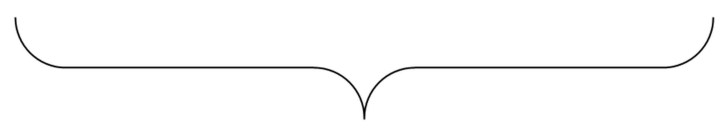

Calcium Channel Antagonist Activity

Figure 13 Calcium-channel antagonists. 
<smiles>[R]C1C(C(=O)NNC(N)=S)=C(C)N=C(C)C1C(=O)NNC(N)=S</smiles>

Figure I $4 \mathrm{~N}$-aryl-I,4-dihydropyridines containing thiosemicarbazone.<smiles>COCc1c(C(C)C)nc(-c2ccc(F)cc2)c(/C=C/[C@@H](O)C[C@H](O)CC(=O)O)c1-c1ccc(F)cc1</smiles>

Figure 15 Cholesterol-lowering drugs in the statin class.

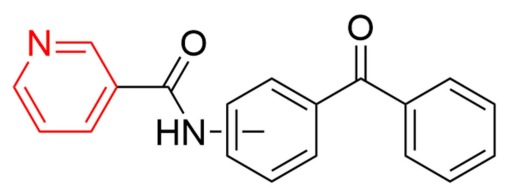

\section{Lipid Profile}

\begin{tabular}{lll}
\cline { 2 - 3 } & \multicolumn{1}{c}{ TG $(\mathrm{mg} / \mathrm{dL})$} & $\mathrm{TC}(\mathrm{mg} / \mathrm{dL})$ \\
\cline { 2 - 3 } 3-NH = 16: & $76.0 \pm 20.1$ & $49.7 \pm 12.7$ \\
4-NH=17 $:$ & $72.2 \pm 9.4$ & $66.0 \pm 7.6$
\end{tabular}

Figure 16 Antihyperlipidemic (benzoylphenyl)pyridine-3-carboxamide compounds.

Pseudomonas aeruginosa, S. aureus, Staphylococcus epidermidis, Escherichia coli, Bacillus cereus, and Gram-positive bacteria showed greater inhibitory activity than Gram-negative bacteria. Most of the synthesized compounds were highly potent against $S$. aureus and $S$. epidermidis. Compounds $\mathbf{4 1}$ and $\mathbf{4 2}$ had strong antibacterial effects with excellent MIC and selectivity-index values (Figure 23). The key point in this study was the single-step synthesis of oxadiazole-pyridine derivative. This approach with sufficient molecular modification could be adopted as a cost-effective strategy to produce potent antimicrobial agents. ${ }^{190}$
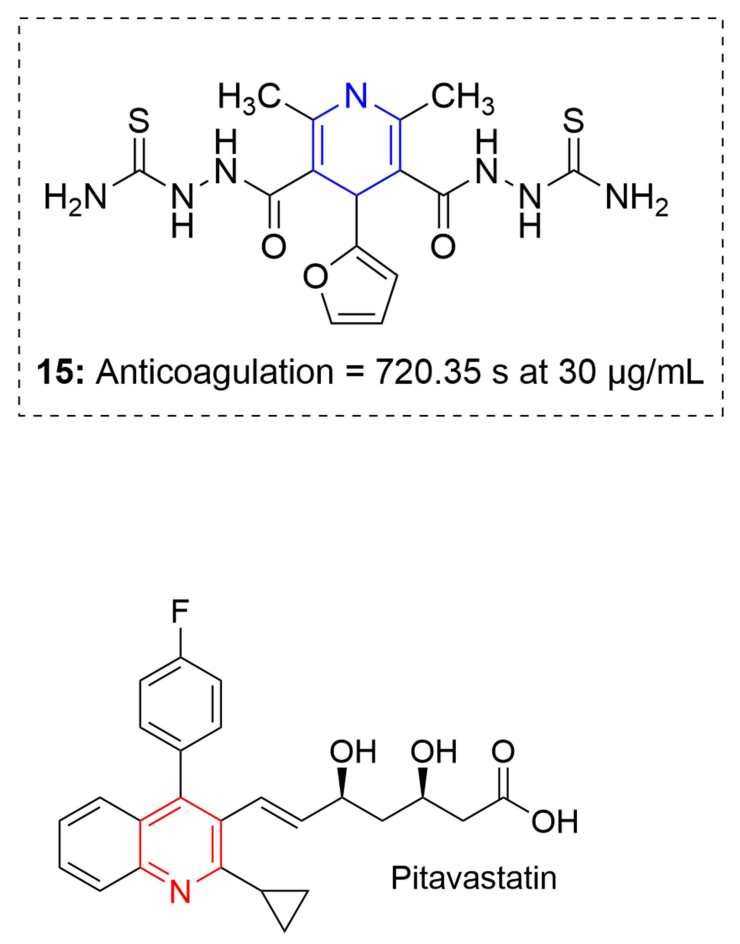

In another study, 1,3,4-oxadiazole derivatives containing indole and pyridine waere synthesized and evaluated against two strains of Mycobacterium tuberculosis - H37Ra and BCG - both in dormant and active conditions. Compound 43-45 showed remarkable antitubercular activity (Figure 24). Antiproliferative activity of the synthesized compounds weas also tested on HeLa, PANC1, and A549 cell lines using modified MTT assays. Most were acytotoxic. Based on MIC values and cytotoxicity results, the selectivity-index values determined for $\mathbf{4 3}-\mathbf{4 5}$, which were highly potent against Mycobacterium bovis BCG, while the compounds had index values $\geq 10$. In addition, molecular docking studies were performed at the active site of enoyl reductase (InhA) for compounds 43-45. The encouraging results substantiated by selectivity, potency, and low cytotoxicity indicate these derivatives as potential antitubercular lead agents. ${ }^{9}$

In the FDA database, one can find many pyridinecontaining drugs, such as isoniazid, ethionamide, and prothionamide, that are highly effective against mycobacteria for the treatment of tuberculosis (Figure 25).

Further efforts to discover new bioactive compounds resulted in the synthesis of 2-(1-adamantylthio) pyridine 


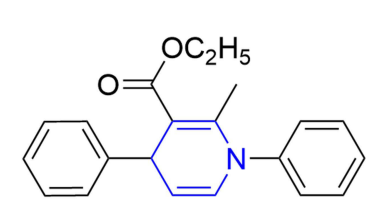

18

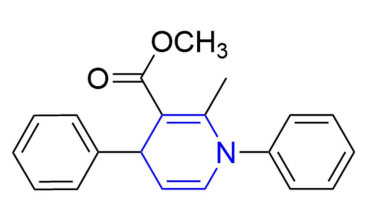

19

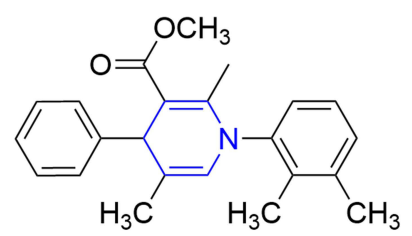

20

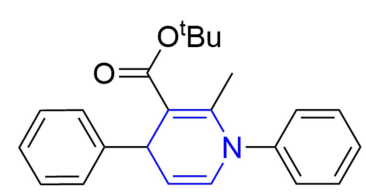

21<smiles>CCCCOC(=O)C1=C(C)N(c2ccc(Cl)cc2)C=CC1c1ccccc1</smiles>

22

Figure 17 Cholesterol-lowering compounds (18-22) containing dihydropyridine rings.<smiles>CCO/N=C(/C(=O)N[C@H]1C(=O)N2C(C(=O)[O-])=C(Sc3nc(-c4cc[n+](C)cc4)cs3)CS[C@H]12)c1nsc(NP(=O)(O)O)n1</smiles>

Ceftaroline fosamil (2010)

Tedizolid (2014)<smiles>CC(C)(O/N=C(/C(=O)N[C@H]1C(=O)N2C(C(=O)[O-])=C(C[n+]3ccccc3)CS[C@H]12)c1csc(N)n1)C(=O)O</smiles>

Ceftazidime (2015)

Delafloxacin (2017)

Figure 18 Pyridine-containing antibiotics approved by the FDA during the last decade. 


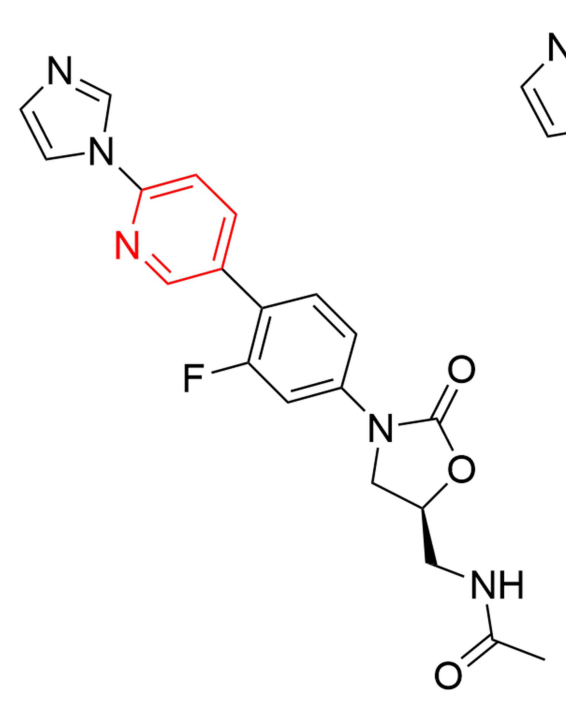

23

Moraxella Catarrhalis $=1.56$

Haemophilus influenzae $=6.25$

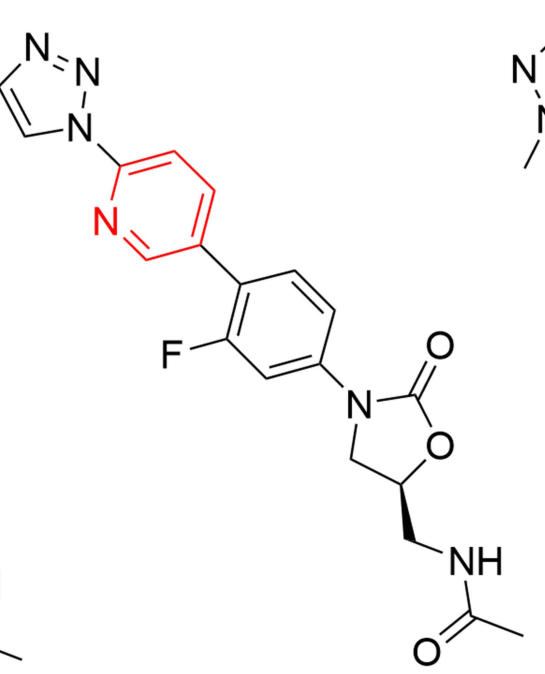

24

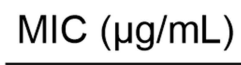

1.56

3.13

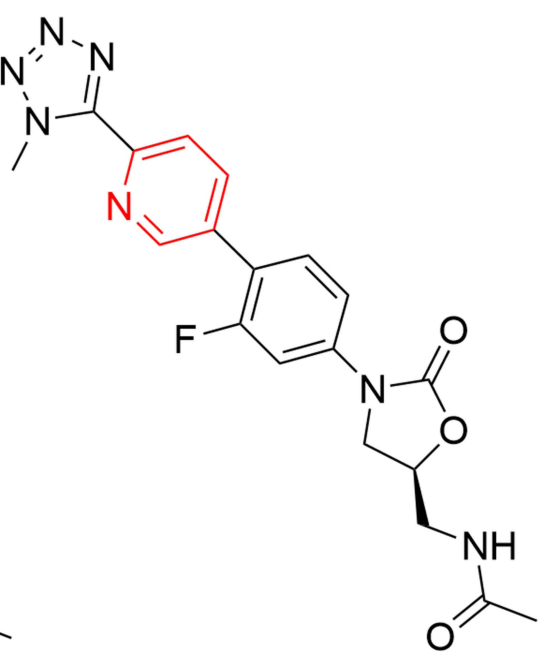

25

$\operatorname{MIC}(\mu \mathrm{g} / \mathrm{mL})$

1.56

3.13

Figure 19 Oxazolidinone-pyridine-substituted antibacterial agents.

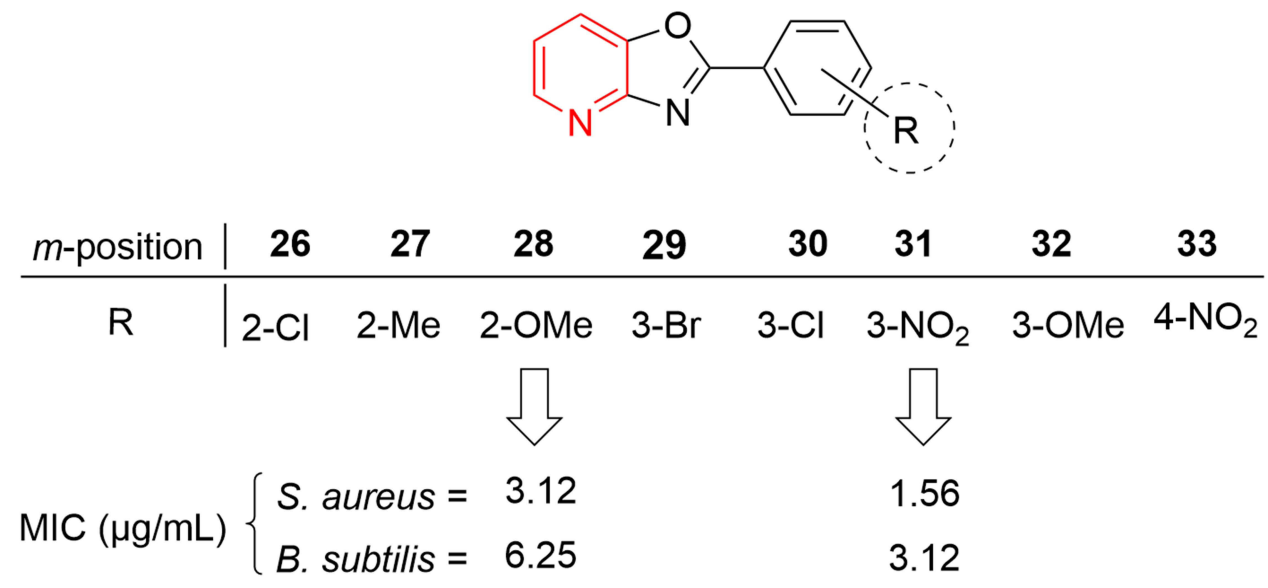

Figure 20 Oxazolo[4,5-b]pyridines containing antibacterial agents with remarkable activity.

derivatives, which were screened for antibacterial activity against 27 strains, antimalarial activity against Plasmodium falciparum, and anticancer activity against HepG2, A549, HuCCA1, and MOLT3 cell lines. The results suggested that 2-(1-adamantylthio)pyridine-type compounds constituted a new class of antibacterial, antimalarial, and anticancer agent with potential therapeutic applications. All the compounds were highly active against streptococci, showing antigrowth activity of $15-30 \mu \mathrm{g} / \mathrm{mL}$. Compounds 46-49 were potent antimalarial, anticancer, and antibacterial agents (Figure 26). Surprisingly, 6-(1adamantylthio)nicotinonitrile 49 had selective antimicrobial activity against $\beta$-hemolytic streptococcus, Edwardsiella tarda, Vibrio parahaemolyticus, and Vibrio cholerae. These findings suggest that compound $\mathbf{4 9}$ could be a promising antibacterial agent with potential ofor further improvement in its therapeutic properties. ${ }^{191}$

In summary, pyridine-containing compounds hold great promise for the development of pharmaceuticals against drug-resistant bacteria, since they exert 


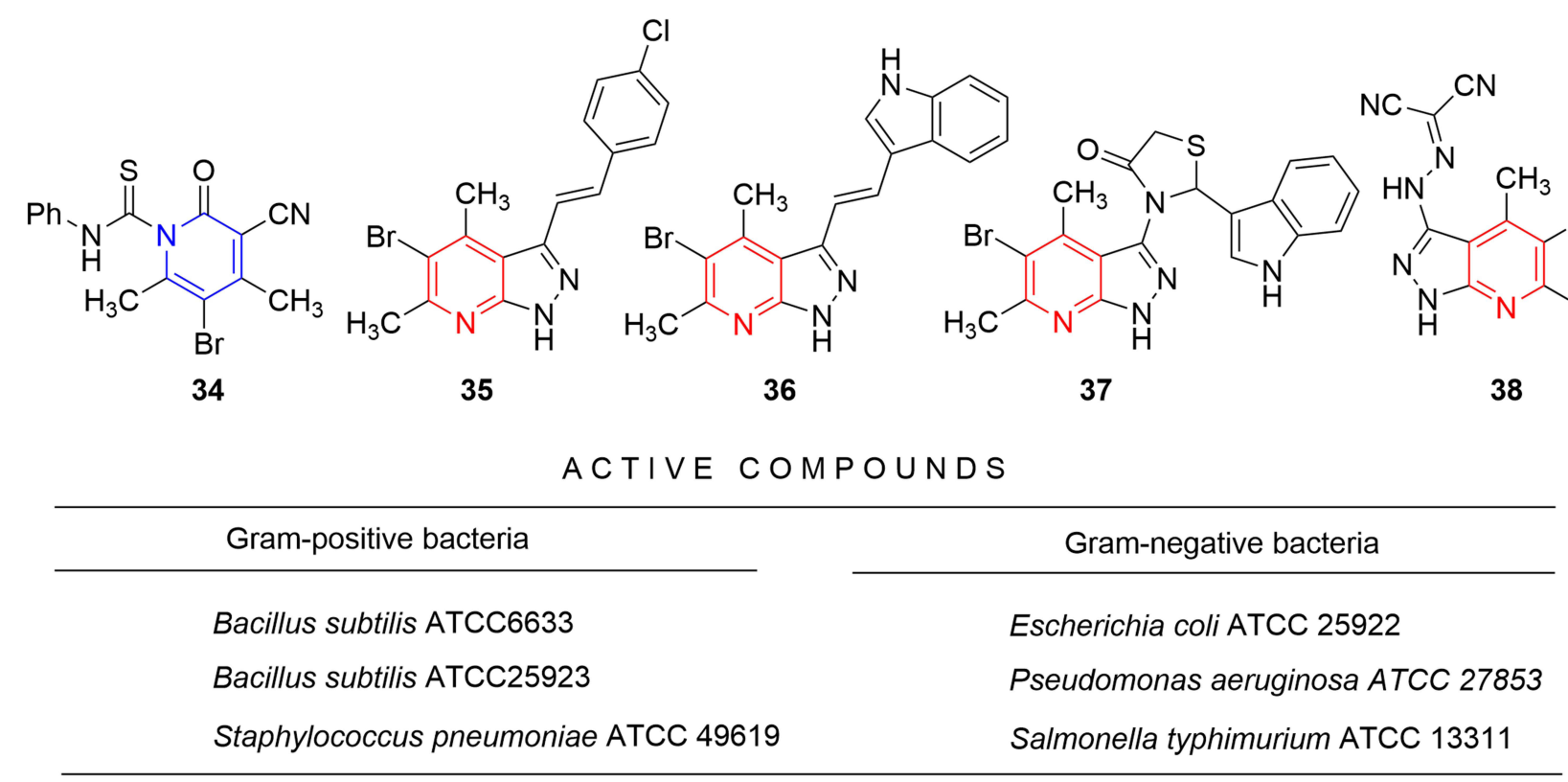

Figure 2 I Pyrazolo[3,4-b] pyridine-bearing compounds with significant effect against various Gram-positive and Gram-negative bacterial strains.<smiles>Cc1csc(Nn2c(C)cc(C)c(C#N)c2=O)n1</smiles>

39<smiles>Cc1cc(C)n(Nc2nc(-c3cc4ccccc4oc3=O)cs2)c(=O)c1C#N</smiles>

40
MIC $(\mu \mathrm{g} / \mathrm{ml})$
$93.7 \pm 0.95$
$93.7 \pm 0.95$
$62.5 \pm 2.00$
$7.8 \pm 0.17$
$5.8 \pm 0.65$

Figure 22 Antibacterial dihydropyridines with thiazole moiety.<smiles>OC(c1ccccn1)c1nnc(-c2ccccc2)o1</smiles><smiles>OC(c1ccccn1)c1nnc(-c2ccc3ccccc3c2)o1</smiles>

41 < Active against Gram-positive bacteria > 42

Figure 23 Highly potent antibacterial agents against staphylococcal infections.

significant inhibitory effects on pathogens. However, more research is needed to find a viable solution to drug-resistant pathogens.

\section{Antifungal Agents}

The recent rise in multidrug-resistant (MDR) fungal infections has led researchers to find new antifungal agents. 


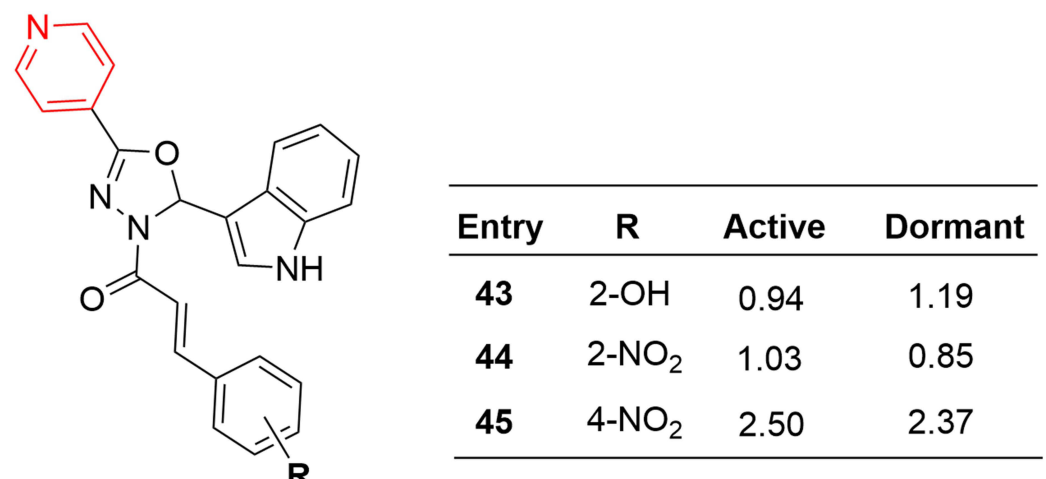

43-45

Figure 24 Highly potent antitubercular compounds (43-45) with MIC values $(\mu \mathrm{g} / \mathrm{mL})$ against $M$. bovis BCG.<smiles>NNC(=O)c1ccncc1</smiles>

Isoniazid<smiles>[R]c1cc(C(N)=S)ccn1</smiles>

Ethionamide $(\mathrm{R}=$ Ethyl $)$

Prothionamide $(\mathrm{R}=$ Propyl)

Figure 25 Pyridine-containing drugs against mycobacteria.

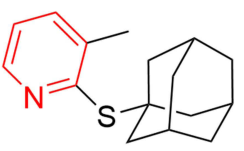

46

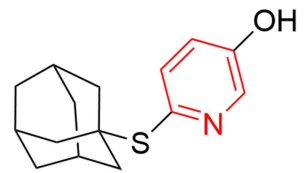

47

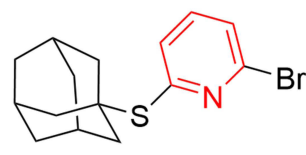

48

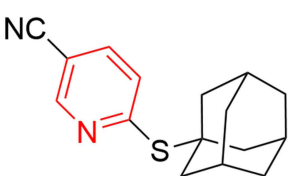

49

Figure 262 (I-adamantylthio) pyridine derivatives with potent antimicrobial activity.

Pyridine-containing triazolo derivatives demonstrate remarkable antifungal properties. For example, thiadiazole-containing triazolopyridines have been found to display antifungal activity against Pseudoperonospora cubensis, Pseudomonas syringae pv. Lachrymans, and Corynespora cassiicola. ${ }^{192}$ In 2016, Mu et al reported a series of hydrazone-containing triazolopyridine derivatives with significant antifungal activity against Stemphylium lycopersici, Botrytis cinerea, and F. oxysporum. ${ }^{193}$ In 2019, Wei et al designed inulineSchiff bases bearing pyridine rings and evaluated their in vitro antifungal properties against Phomopsis asparagi, F. oxysporum f. sp. niveum, and Botrytis cinerea. ${ }^{194}$ Pyridinegrafted chitosan polymers have also been reported with improved antifungal properties. ${ }^{195}$ Pyridine has beeno grafted onto starch to control different fungi. ${ }^{196}$ In summary, fusing triazole and pyridine derivatives may lead to the development of broad-spectrum antifungal agents against MDR fungal infections.

\section{Antimalarial Agents}

A series of novel pyridyl-indole hybrids were described by Heba et al, which were designed using a fragmentbased strategy. The compounds were tested for antimalarial activity against chloroquine-sensitive (D6) and chloroquine-resistant (W2) strains of $P$. falciparum. Compounds 50-55 (Figure 27) displayed the most potent antimalarial activity $\left(\mathrm{IC}_{50}=1.47-9.23 \mu \mathrm{M}\right.$ for $\mathrm{D} 6$ and $\mathrm{IC}_{50}=1.16-7.66$ $\mu \mathrm{M}$ for W2). Selectivity-index values were 1.47-8.3 for D6 and 1.7-10 for W2. Compounds 50, 51, and 54 demonstrated antimalarial activity against D6 and W2. The distinctive feature of these compounds was the absence of substitution at the $\mathrm{C} 2$ position of the pyridine ring. 


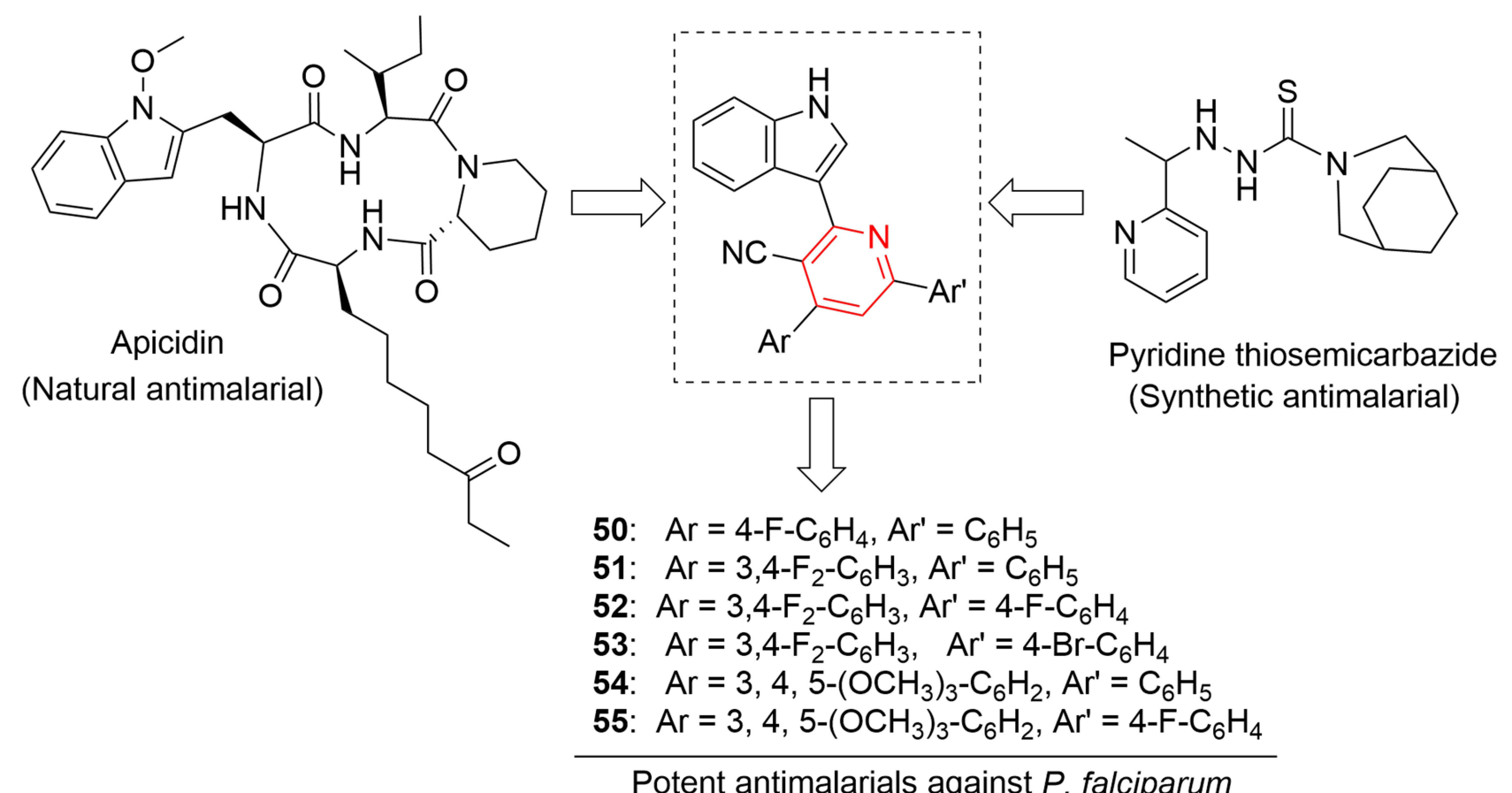

Figure 27 Highly active antimalarial pyridyl-indole hybrids.<smiles>[R]C(=O)N(O)CCC(c1ccncc1)P(=O)(O)O</smiles>

\section{$P$. falciparum DXR Ki $=1.9 \mathrm{nM}$ \\ $P$. falciparum killing $\mathrm{EC}_{50}=170 \mathrm{nM}$}

Figure 28 Highly potent antimalarial pyridine-containing fosmidomycin derivative.

Furthermore, binding interactions of these compounds with quadruple mutant $P$. falciparum dihydrofolate reductase enzyme were investigated via molecular docking studies. Compounds 50-52 were the most active at the binding cavities of quadruple-mutant Pf DHFR-TS-active sites, indicating suitable binding associations that might be the mechanism influencing their activity as antimalarial agents. ${ }^{197}$

Xue et al reported fosmidomycin derivatives containing the pyridine scaffold that inhibited P. falciparum DXR with $\mathrm{K}_{\mathrm{i}}$ values of 1.9-13 nM. The most potent compound (Figure 28) was elevenfold as active as fosmidomycin. ${ }^{198}$

We believe that pyridine-based compounds have immense potential for the development of antimalarial drugs, since they exhibit antimalarial effects due to hydrogen-bond interactions between pyridine nitrogen and cysteine of target proteins in the pathogen, thereby rendering such compounds highly effective against chloroquineresistant strains. ${ }^{198}$

\section{Antiviral Agents}

For the treatment of HIV infection, the FDA data-base contains many pyridine- and dihydropyridine-containing drugs, such as nevirapine, tipranavir, doravirine, and indinavir (Figure 29).

In the last decade, research into new antiviral agents has resulted in the synthesis of pyridotriazines, furopyridines, and pyridothiadiazepinthiones. ${ }^{199}$ Among the synthesized ones, a few molecules had appreciable efficacy against adenovirus type 7 and the rotavirus Wa strain. Compound $\mathbf{5 6}$ suppressed viral titers by $60 \%$ and $53.3 \%$ for the rotavirus Wa strain and adenovirus type 7, respectively, and compound 57 demonstrated $50 \%$ and $53.3 \%$ reductions, respectively. These compounds (Figure 30) can potentially be used as therapies for rotavirus and adenovirus type 7, which currently have no adequate treatment options. ${ }^{199}$ 


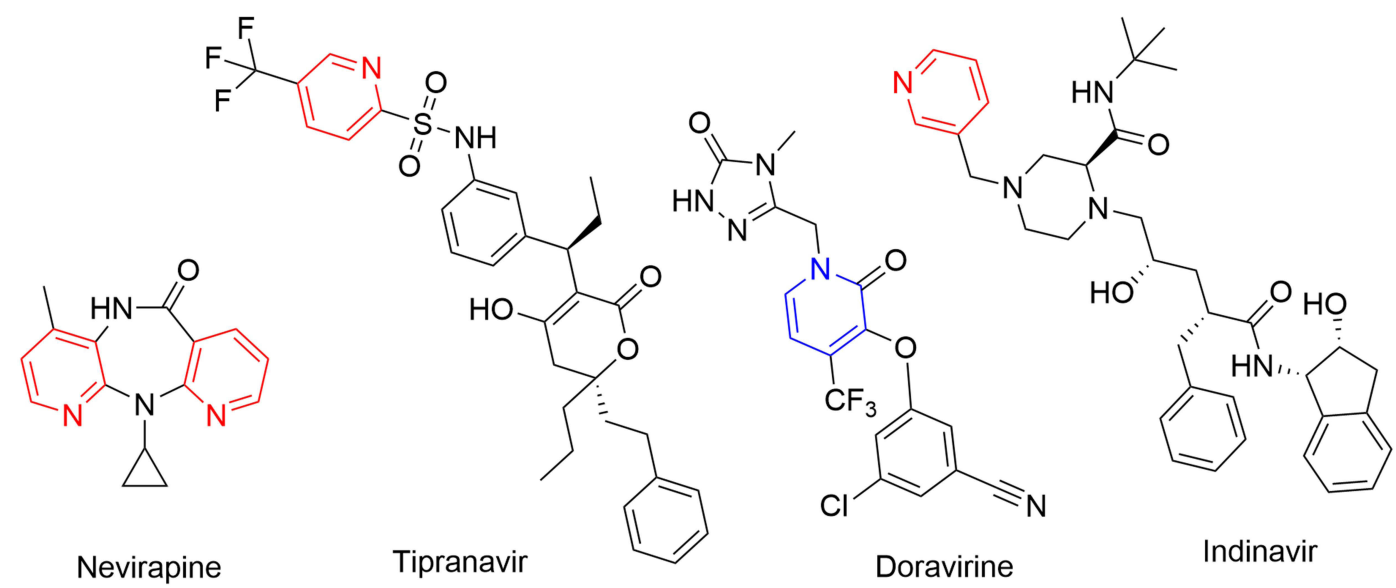

Figure 29 Pyridine/dihydropyridine-containing drugs in the market for HIVIAIDS treatment.<smiles>CCOC(=O)c1oc2nc(-c3cccc4ccccc34)cc(-c3ccco3)c2c1N</smiles>

56<smiles>N#Cc1c(-c2ccco2)cc(-c2cccc3ccccc23)nc1N</smiles>

57

Figure 30 Pyridine-furan hybrid compounds with $50 \%$ reduction in viral titer against adenovirus 7 strain.<smiles>C#CCOc1nc(-c2ccc3ccccc3c2)cc(-c2cccs2)c1C#N</smiles>

Figure 3 I Potent antiviral compound $\mathbf{5 9}$ with activity against $\mathrm{H} 5 \mathrm{NI}$ influenza virus.

In recent years, antiviral drugs have been developed as a result of the search for viable viral therapeutic approaches. Click chemistry is one of the most effective ways for producing bioorganic molecules, such as antiviral therapeutics. In a recent study, pyridine derivatives were acquainted with propargyl group by $O$-propargylation 58-59 (Figure 31). Cu-catalyzed cycloaddition of azido-sugars with substituted (propargyl)oxypyridines or propargyl sugars with azidoethoxypyridine derivatives resulted in high yields of desired 1,2,3-triazoles. MTT and plaque-reduction assays were performed against the $\mathrm{H} 5 \mathrm{~N} 1$ influenza strain to evaluate antiviral activity. High activity and low toxicity were demonstrated by triazolyl glycoside 58. The effect of pyridinyl fragment binding to glycosyl triazole moieties on antiviral activity was studied using SAR correlations. Most of the compounds had weakly active to moderately active inhibitory profiles at different concentrations, with the exception of compound 59, which had the strongest activity. All tested compounds showed dosedependent inhibitory behavior. Low cytotoxicity was observed for compounds $\mathbf{5 8}$ and $\mathbf{5 9}{ }^{200}$ 
<smiles>COc1cc(Nc2ccnc3ccc(Br)cc23)cc(OC)c1OC</smiles>

SGC-GAK-1 60

GAK $K_{D}=1.9 \mathrm{nM}$

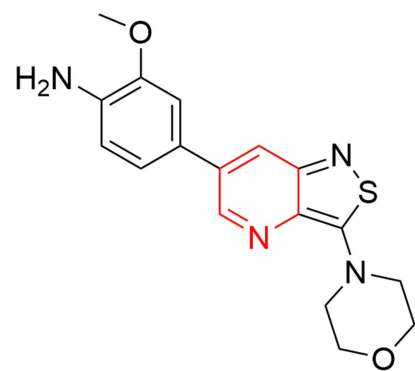

61

GAK $K_{D}=8.9 \mathrm{nM}$<smiles>COc1cc(-c2cnc3c(N4CCOCC4)snc3c2)cc(OC)c1OC</smiles>

62

GAK $K_{D}=8.3 \mathrm{nM}$

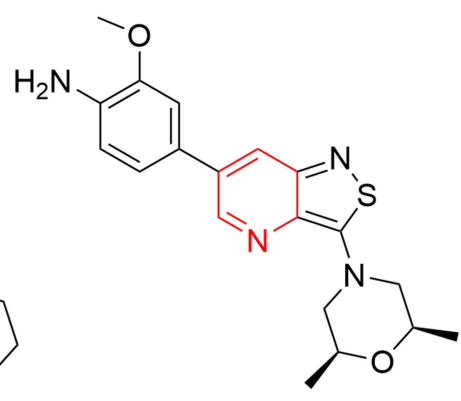

63

GAK $K_{D}=89 \mathrm{nM}$

Figure 32 Antiviral GAK inhibitors containing isothiazolopyridine scaffold.

Auxilin 2, also known as cyclin $\mathrm{G}$-associated kinase (GAK), has been demonstrated to impact both the initial and late phases of the viral life cycle, thereby functioning as the master regulator against viral infection. This host-specific strategy offers many advantages, such as development of broad-spectrum antiviral agents and high barriers to resistance. Asquith et al initially discovered SGC-GAK-1 60, which has excellent GAK affinity with a $\mathrm{K}_{\mathrm{D}}$ value of $1.9 \mathrm{~nm} .^{201}$ Later, Jonghe et al developed potent and selective GAK inhibitors 61-62 that were basically isothiazolopyridine-type compounds with morpholine residue (Figure 32). These compounds had high GAK affinity, but were moderately active against dengue and hepatitis $\mathrm{C}$ viruses. ${ }^{202}$ Subsequent research showed that the introduction of dimethyl groups to the morpholine residue of $\mathbf{6 2}$ had favorable antiviral effects, which led to the discovery of $\mathbf{6 3}$. The compound was active against chikungunya, dengue, and Zika viruses. ${ }^{203}$ However, replacing the morpholine residue with carboxamides, alkoxides, and amines resulted in weak antiviral effects. ${ }^{204,205}$

Further modifications to the pyridine core of $\mathbf{6 3}$ were done to further improve the antiviral effect. The new compounds were highly active against dengue, with GAK-binding affinity<smiles></smiles><smiles>COc1ccc(-c2cnc3c(N4C[C@H](C)O[C@H](C)C4)snc3c2)cc1OC(C)(C)O[Mg]</smiles>

Figure 33 Antiviral compounds capable of targeting cyclin G-associated kinase of dengue virus.

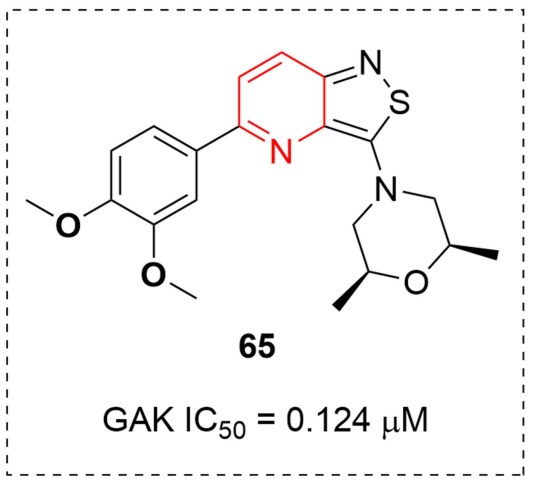<smiles>COc1ccc(-c2ccc3nsc(N4CCOCC4)c3n2)cc1OC</smiles>

5-folds less active than 65

Figure 34 Antiviral compounds with high GAK-binding affinity. 
in the nanomolar range. For example, compound 64 inhibited GAK strongly (Figure 33) and was equally potent against dengue. $^{206}$

A novel series of isothiazolopyridines containing 3.4dimethoxyphenyl residue was also synthesized, ${ }^{206}$ wherein $\mathrm{GAK} \mathrm{IC}_{50}$ values were $0.1-0.5 \mu \mathrm{M}$. Compound $\mathbf{6 5}$ had the highest GAK affinity $\left(\mathrm{IC}_{50}=0.124 \mu \mathrm{M}\right)$, whereas compound 66 containing $N$-morpholinyl residue was fivefold less active (Figure 34). Diverse structural modifications at the active site of the 6-phenyl moiety's position 4 can, however, be employed to alter antiviral activity. ${ }^{206}$

Although many pyridine-containing drugs are commercially available to manage HIV/AIDS, further research on the isothiazolopyridine class of compounds could lead to a viable solution for other types of viral infections, such as dengue.

\section{Anti-inflammatory Drugs and Active Compounds}

Oxicam compounds are used to treat musculoskeletal disorders: acute and chronic inflammation via inhibition of the cyclooxygenase isoforms COX1 and COX2. ${ }^{207}$ The FDA-approved oxicam NSAIDs containing the pyridine moiety are shown in Figure 35. These drugs are mainly used to treat musculoskeletal disorders, such as osteoarthritis and rheumatoid arthritis, by relieving painful inflammatory conditions. ${ }^{208}$
Clonixin (Figure 36) is another FDA-approved drug and has analgesic and antipyretic effects in chronic arthritic conditions. ${ }^{69}$ For etoricoxib, the FDA needs additional safety data for the approval. It is, however, licensed in $>80$ countries worldwide. The drug, which is a COX2 inhibitor, is mainly used for the treatment of gout, ankylosing spondylitis, osteoarthritis, psoriatic arthritis, and rheumatoid arthritis. $^{209}$

Recently, many bioactive molecules have been reported to deal with inflammatory markers. ${ }^{210,211}$ Thirumurugan et al synthesized indole-bearing pyridine derivatives and evaluated their anti-inflammatory activity against rat-paw edema. All compounds had remarkable anti-inflammatory activity, particularly 67-68, which demonstrated significantly higher activity than the standard drug indomethacin (Figure 37). The analgesic activity of dihydropyridine derivatives was also compared with aspirin. Compounds 68-70 had considerably higher analgesic activity. ${ }^{212}$

In an attempt to broaden the scope of anti-inflammatory research, Liu et al designed thienopyridine derivatives (Figure 38). When subjected to NO-production assays, most of the compounds were able to inhibit NO production. The most effective analogue, $\mathbf{7 2}$, substantially reduced NO production at lower doses $\left(\mathrm{IC}_{50}=3.30 \mu \mathrm{M}\right)$. Anti-inflammatory profiles were further investigated by evaluating $\mathrm{TNF} \alpha$-inhibitory activity of the most potent compound -<smiles>CCOC(=O)OC(C)OC1=C(C(=O)Nc2ccccn2)N(C)S(=O)(=O)c2ccccc21</smiles>

Figure 35 FDA-approved oxicam-class NSAIDs for musculoskeletal disorders, such as osteoarthritis and rheumatoid arthritis.

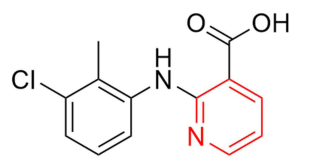

Clonixin (FDA approved drug)

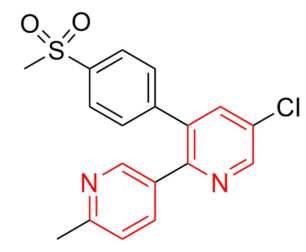

Etoricoxib (not FDA approved) (Licensed in the EU)

Figure 36 Commercially available NSAIDs containing the pyridine ring. 
<smiles>COc1nc(-c2c[nH]c3ccccc23)c(C#N)c(-c2ccc(Br)cc2)c1C#N</smiles><smiles>COc1nc(-c2c[nH]c3ccccc23)c(C#N)c(-c2ccc(F)cc2)c1C#N</smiles>

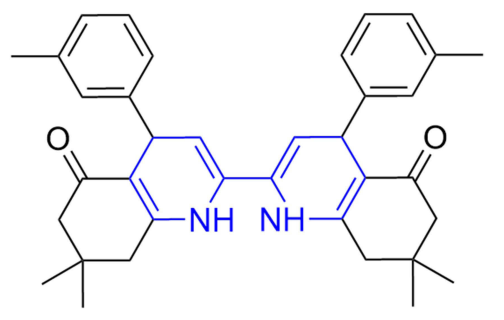

69

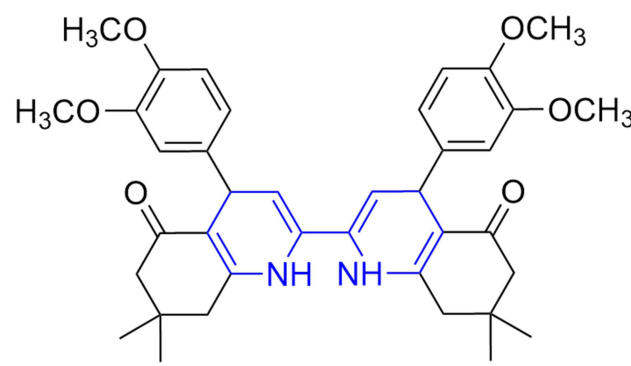

70

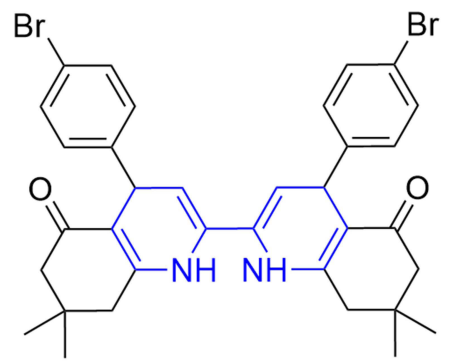

71

Figure 37 Indolyl pyridines (67-68) and dihydropyridine-containing compounds (69-71) with remarkable anti-inflammatory activity in animal models.<smiles>[R]c1ccc2c(N)c([R])sc2n1</smiles>

\begin{tabular}{l|ll|c} 
& \multicolumn{1}{|c}{$\mathrm{R}_{1}$} & $\mathrm{IC}_{50}(\mu \mathrm{M})$ \\
\hline 72 & 3-MeO-Ph & $-\mathrm{CONH}_{2}$ & 17.7 \\
73 & 3-F-Ph- & $-\mathrm{CONH}_{2}$ & 3.24 \\
74 & 3-F-Ph & $N-(4-(4-M e t h y l p i p e r a z i n-1-y l) p h e n y l) N H C O-$ & 7.46 \\
75 & 3-MeO-Ph & $N-(4-(4-M e t h y l p i p e r a z i n-1-y l) p h e n y l) N H C O-$ & 5.80
\end{tabular}

Figure 38 Thienopyridine derivatives (72-75) with anti-inflammatory and immunomodulatory profiles. $I_{50}$ values correspond to inhibition of NO production on murine RAW264.7 macrophages.

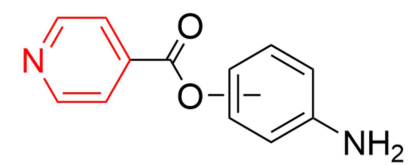

76: $3-\mathrm{O}$

77: $4-\mathrm{O}$

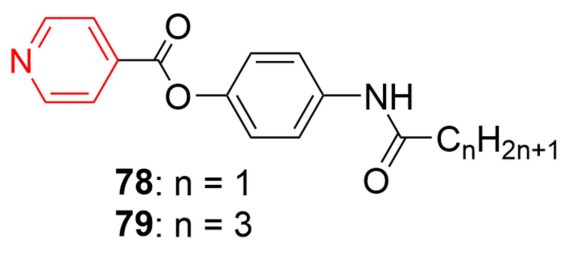

79: $n=3$

Figure 39 Highly potent anti-inflammatory compounds. 
72. Interestingly, compounds with piperazine residue $\mathbf{- 7 4}$ and 75 - demonstrated comparable effectiveness. These results indicated that thienopyridine-containing compounds of 72-75 may represented a new class of anti-inflammatory drugs that necessitated more attention. ${ }^{213}$

Yaqoob et al recently reported on highly potent antiinflammatory compounds designed by employing pyridinecontaining isonicotinic acid (Figure 39). Remarkable ROSinhibitory activity was observed for compounds 76-79. Compound 76 was one of the most potent anti-inflammatory agents, with $\mathrm{IC}_{50}$ of $1.42 \pm 0.1 \mu \mathrm{g} / \mathrm{mL}^{214}$

The antagonizing hormone glucocorticoid stimulates hepatic glucose synthesis while inhibiting insulinassisted glucose absorption in skeletal muscles and adipose tissue. ${ }^{215}$ Glucocorticoidal stimulation, which is coordinated by $11 \beta$-HSD2 and $11 \beta-H S D 1$ enzymes, is often used to measure glucocorticoid target-tissue activity. ${ }^{216}$ Enzyme 11-HSD1 is considered to play a crucial role during lipid and glucose metabolism in adipose tissue. Therefore, inhibitors of $11-\mathrm{HSD} 1$ are a novel family of drugs that are being developed to address diabetic complications. The role of 11-HSD1 in the development of insulin resistance and obesity has been shown in several preclinical investigations. Recently, $\alpha$-glucosidase-inhibitory activity was tested in vitro using a new set of triazole-containing dihydropyridine derivatives. When compared to the acarbose standard $\left(\mathrm{IC}_{50}=395.17 \mu \mathrm{M}\right)$, these compounds showed considerable $\alpha$-glucosidase-inhibitory activity $\left(\mathrm{IC}_{50}=\right.$ 72.71-283.41 $\mu \mathrm{M}$ ). Compounds 80-82 (Figure 40) seemed to have the highest inhibitory action against the enzyme, with $\mathrm{IC}_{50}$ values of $72.71 \pm 1.09,73.83$ \pm 1.17 , and $85.96 \pm 1.84 \mu \mathrm{M}$, respectively. To understand the mechanism of action, the most efficient compounds (80 and 81) of the series were evaluated using in vitro enzymatic tests to assess their 11 $\beta$-HSD1 enzyme-inhibitory activity. The mechanism of action of $\mathbf{8 0}$ and $\mathbf{8 1}$ was further confirmed using molecular docking analysis, which showed that both compounds bound strongly in the cavity of $11 \beta$-HSD1 receptors, resulting in appreciable dock scores, electrostatic energy, and hydrogenbond interactions for the desired molecular complex (both sides and back chain). Overall, compounds $\mathbf{8 0}$ $(-9.758)$ and $81(-8.595)$ demonstrated highly stable binding patterns for $11 \beta$-HSD1 in molecular docking studies. $^{217}$

Larijani et al reported excellent $\alpha$-glucosidase activity for coumarin-fused pyridines (Figure 41). Most of their compounds had $\mathrm{IC}_{50}$ values in the range of $101.0 \pm 2.0$ to $227.3 \pm 1.4 \mu \mathrm{M}$, whereas the standard drug acarbose had an $\mathrm{IC}_{50}$ value of $750.0 \pm 1.5 \mu \mathrm{M}$. Compounds 83-85 were the most potent, with $\mathrm{IC}_{50}$ values of $101.0 \pm 2.0,111.3 \pm 1.5$ and $114.3 \pm 1.8 \mu \mathrm{M}$, respectively. ${ }^{218}$

Although many pyridine-containing NSAIDs are commercially available for musculoskeletal disorders, such as osteoarthritis and rheumatoid arthritis, further research on

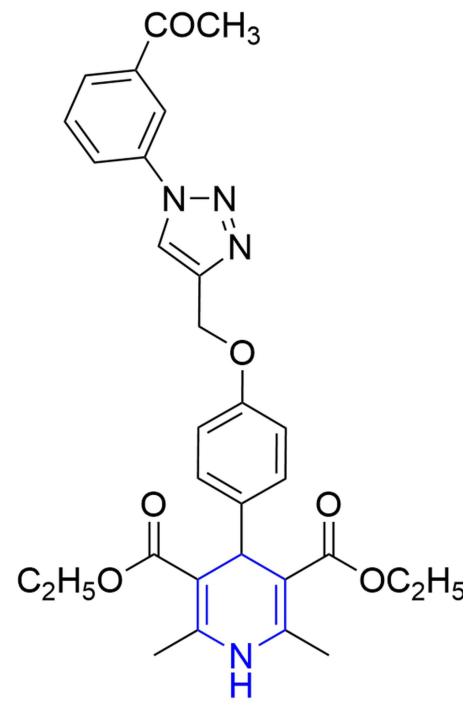

$80(98.3 \%)$

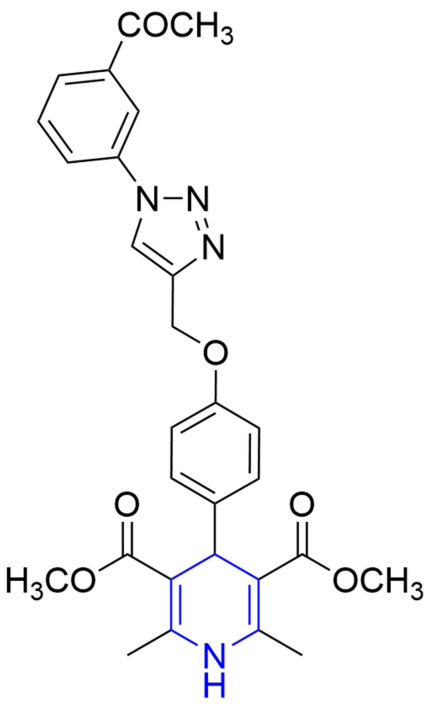

$81(97.5 \%)$<smiles>CCOC(=O)C1=C(C)NC(C)=C(C(=O)OCC)C1c1ccc(OCc2cn(-c3cccc(Cl)c3)nn2)cc1</smiles>

$82(96.8 \%)$

$\%$ inhibition of $\alpha$-glucosidase activity

Figure 40||$\beta-H S D \mid$ inhibitors against diabetes mellitus. 


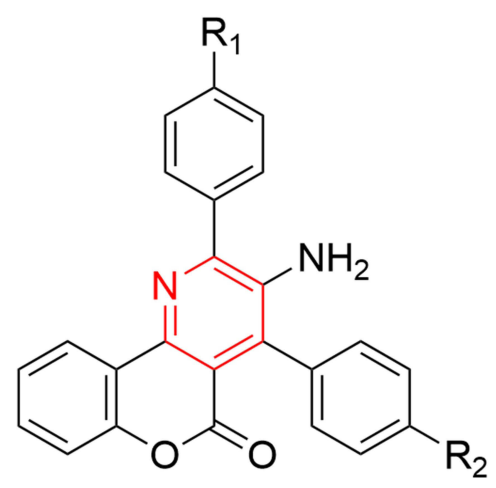

83: $R_{1}=B r, R_{2}=C H 3, I C_{50}=101.0 \pm 2.0 \mu M$

84: $R_{1}=B r, R_{2}=H, I C_{50}=111.3 \pm 1.5 \mu M$

85: $\mathrm{R}_{1}=\mathrm{CH}_{3}, \mathrm{R}_{2}=\mathrm{Cl}, \mathrm{IC}_{50}=114.3 \pm 1.8 \mu \mathrm{M}$

Figure 4I Coumarin-fused pyridines with potent $\alpha$-glucosidase activity.

pyridine- or dihydropyridine-containing oxicams may lead to the development of effective drugs for the cure of acute and chronic inflammation.

\section{Neurogenic Drugs and Bioactive Compounds}

Neuroprotection may be defined as the maintenance, preservation, and stability of neuronal functions and structures. It is a mechanism for smooth working of the nervous system and prevention of neural damage. ${ }^{219}$ The brain is a very sensitive part of body, so remains highly vulnerable to pathogens and damage causing neurodegenerative disorders, such as Parkinson's disease, amyotrophic lateral sclerosis, epilepsy, brain tumors, and Alzheimer's disease. ${ }^{220,221}$ These illnesses are main cause of neuronal death, including neural strokes, which are a result of different complications like calciumhomeostasis loss, cytotoxicity, metabolic failure, and oxidative stress. ${ }^{222}$ Many pyridine- or dihydropyridine-containing drugs are being evaluated for the treatment of neurodegenerative disorders ${ }^{23}$ (Figure 42).

\section{Alzheimer's Disease}

Nimodipine is a wide-spectrum neuroprotective drug that is widely used in Alzheimer's disease, migraine, and posthemorrhagic vasospasm as an anti-ischemic agent. ${ }^{224}$ Nimodipine (Figure 43) is well known for its relaxing potential for cerebral vasculature. ${ }^{225}$

Alzheimer's disease is a neurodegenerative disorder characterized by memory dysfunction and cognitive impairment. A number of compounds based on pyridine and dihydroxypyridine have been synthesized and evaluated for their anti-Alzheimer's activity. León et al developed a series of tacrine-dihydropyridine hybrids decorated with pyrin scaffolds. ${ }^{226}$ The series was evaluated for inhibitory potential against acetylcholinesterase. Compound 86 had tenfold the activity $\left(\mathrm{IC}_{50}=0.0048 \pm 0.001\right)$ of the donepezil standard $(0.049 \pm 0.005$; Figure 44$)$.

Huperzine A 94 (Figure 45) is well recognized for its neuroprotective properties, which result in enhanced NGF production and expression, which are involved in the functional enhancement of neurons, their survival, and protection against damage in neurodegenerative illnesses (such as Alzheimer's). It protects neurons from glutamate toxicity by decreasing glutamate-induced calcium mobilization. It also protects rat pheochromocytoma cells from oxidative stress caused by hydrogen peroxide. Because oxidative stress exacerbates Alzheimer's neurodegeneration, huperzine A 94 is widely used to treat Alzheimer's complications. $^{227}$

Recently, a dihydropyridine derivative with pyridinium moiety $\mathbf{8 7}$ was found to be highly active as a gene-transfection agent and displayed excellent mitochondrion-targeted antioxidant activity. ${ }^{228}$ It was found to play an important role in protection against neural injuy (Figure 46), and causeed increased expression of proteins in the hippocampus and cerebral cortex. It was found that increased expression of the GAD67 enzyme in hippocampus converted the glutamate to GABA, and GABA was found to protect the brain from neural injury. It regulated the development of spatial memory, and via synthesis of GABA it balanced neurotransmitters, consolidation, and stability of memory. Owing to its memory-improvement and neural protection abilities, it can be used in the treatment of Alzheimer's disease.

Dihydropyridine derivatives with pyridinium moiety can be a viable solution for the cure Alzheimer's, since they are able to enhance the expression of crucial proteins in the hippocampus and cerebral cortex. In coming years, extensive research in this type of compound is anticipated.

\section{Parkinson's Disease}

Pyrazoline-containing pyridine derivatives have been reported to display antiparkinsonian activity. ${ }^{229}$ For example, compounds $\mathbf{8 8}$ and $\mathbf{8 9}$ have significant antiparkinsonian activity, with 0.8 relative potency compared to the reference drugs benzatropine and voltaren (Figure 47).

Several dihydropyridines have amino acids in their structure and are peptidomimetic in nature. The most 
<smiles>Cc1ccc(NC(=O)c2ccc(CN3CCN(C)CC3)cc2)cc1Nc1nccc(-c2cccnc2)n1</smiles>

Imatinib

(Under preclinical studies for Alzheimer's)<smiles>Cc1cn(-c2cc(NC(=O)c3ccc(C)c(Nc4nccc(-c5cccnc5)n4)c3)cc(C(F)(F)F)c2)cn1</smiles>

Nilotinib

(Under clinical studies for Parkinson's)<smiles>Cc1ccc(NC(=O)c2ccc(CN3CCN(C)CC3)cc2)cc1Nc1nc(-c2cccnc2)cs1</smiles>

(Under clinical studies for Amyotrophic Lateral Sclerosis)<smiles>Oc1c(I)cc(Cl)c2ncccc12</smiles>

Clioquinol (Under clinical studies for Alzheimer's)<smiles>COC(=O)C1=C(C#N)NC(C)=C(C(=O)OC(C)C)C1c1cccc([N+](=O)[O-])c1</smiles>

Nilvadipine (Under clinical studies for Alzheimer's)

Figure 42 Pyridine- or dihydropyridine-containing drug-repurposing candidates for treatment of neurodegenerative diseases.<smiles>COCCOC(=O)C1=C(C)NC(C)=C(C(=O)OC(C)C)C1c1cccc([N+](=O)[O-])c1</smiles>

Figure 43 Structure of the wide-spectrum neuroprotective drug nimodipine.

studied are glutapyrone $\mathbf{9 0}$ and tauropyrone 91 (Figure 48). Both these compounds protect cerebellar granule cells from damage by lowering lactate dehydrogenase, thus avoiding ischemia/hypoxia (lack of oxygen and glucose) and glutamate excitotoxicity. Tauropyrone 91 can be used for the treatment of Parkinson's disease, as it suppresses the inflammatory process in rats in a Parkinson's 6-hydroxydopamine model at $6.25 \mathrm{~g} / \mathrm{L}$ per day for 7 and 14 days. Tauropyrone $91(1 \mathrm{mg} / \mathrm{kg})$ shows dual actions in the brain. In some cases, it shows inflammatory and proapoptotic effects, but in azidothymidine toxicity, it acts as anti-inflammatory and antiapoptotic agent. The amino acid-containing monocyclic dihydropyridines represent a new atypical group of DHPs, and data have shown their neuromodulatory potential and normalizing effect on protein expression in the brain. ${ }^{230-233}$

Although pyrazoline-containing pyridine derivatives have been reported to display antiparkinsonian activity, 
<smiles>N#Cc1cccnc1N1CCN(C(=O)Nc2n[nH]c3ncccc23)CC1</smiles>

Figure 44 Highly potent AChE inhibitor.

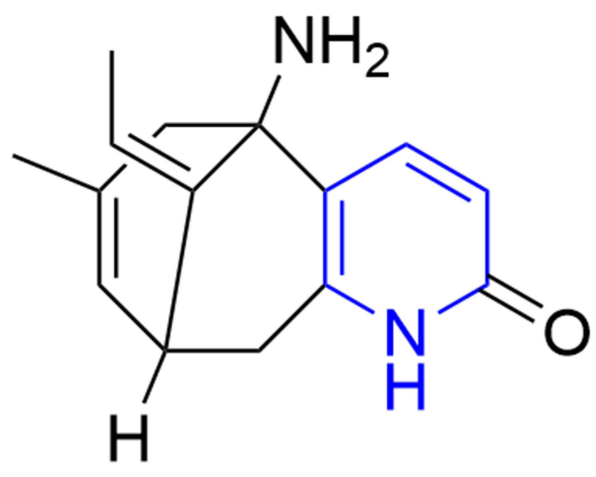

94

Figure 45 Structure of naturally occurring huperzine $A$.<smiles>CCOC(=O)C1=C(C)NC(C)=C(C(=O)OCC)C1c1ccc[n+]([14CH2][18Br])c1</smiles>

\section{7}

Figure 46 Compound 87 is capable of increasing expression of the $\mathrm{GAD}_{67}$ enzyme in the hippocampus.

many reports in the literature have suggested that noncalcium agonistic 1,4-dihydropyridine derivatives demonstrate remarkable neuroprotective effects, thus holding great potential for future drug design against Parkinson's disease.

\section{Cerebral Ischemia}

Cerebral ischemia is also a neuronal disorder in which many noxious by-products and free radicals are generated, resulting in enzymatic activity being altered, which results in a breakdown of cellular phospholipids, proteins, and nucleic acids. Cerebral edema is a complication that occurs due to the overexpression of AQP4 whereby abundant water enters the brain and the swollen brain is compressed against the skull. This increased pressure in the cranium causes herniation and brain ischemia, leading to death. This condition is treated with piroxicam (Figure 49), which has inhibitory effects on AQP4 (the most abundant water channel in the brain). Pyridine ringcontaining piroxicam 92 binds with $\mathrm{AQP} 4$, and in this way regulates it in brain to avoid cerebral ischemia and edema. $^{234}$

\section{Schizophrenia}

Schizophrenia is a mental condition marked by behavioral, neurochemical, and morphological disorders. Antipsychotics that operate on molecular targets other than monoaminergic receptors have not yet been produced, despite significant progress in medication development for schizophrenia. GABAergic dysfunction may be implicated in this disease. ${ }^{235}$ Marcinkowska et al recently discovered the imidazopyridinetype neuroprotective agent 93 (Figure 50), which shows potential affinity for serotonin $5 \mathrm{HT}_{2}$ and $5 \mathrm{HT}_{\mathrm{x}}$ receptors and antipsychotic-like activity. Compound $\mathbf{9 3}$ also shows positive allosteric modulator properties, high metabolic stability, and no hepatotoxicity. ${ }^{235}$

Senna spp. are a celebrated source of natural alkaloids of the piperidine and pyridine classes. ${ }^{236,237}$ Francisco et al isolated five new pyridine-containing alkaloids (Figure 51) from Senna and Cassia spp.: 8'-multijuguinol 95, 7'-multijuguinol 96, methyl multijuguinate 97, 12'hydroxy-8'-multijuguinol 98, and 12'-hydroxy-7'-multijuguinol 99, which were isolated from Senna multijuga leaves. All these compounds had acetylcholinesterase-inhibitory activity comparable to the standard drug physostigmine. SAR studies have suggested that hydroxypyridine moiety is the key interaction site responsible for this activity, whereas the alkyl side chain also influences the acetylcholinesterase-inhibitory effect of the alkaloids. ${ }^{236} \mathrm{~A}$ summary of the neuroprotective compounds with their 
<smiles>COc1ccc(C2CC(c3cccnc3)=NN2C(N)=O)cc1</smiles>

88<smiles>COc1ccc(C2=NC(c3ccccc3)NC(c3cccnc3)C2)cc1</smiles>

89

Figure 47 Antiparkinsonian activity of compounds $\mathbf{8 8}$ and $\mathbf{8 9}$ were comparable to reference drugs.<smiles>CCOC(=O)C1=C(C)NC(C)=C(C(=O)OCC)C1C(=O)N[C@@H](CCC(=O)[OH2+])C(=O)[OH2+]</smiles>

90

Figure 48 Structure of glutapyrone (left) and tauropyrone (right).<smiles>CN1C(C(=O)Nc2ccccn2)=C(O)c2ccccc2S1(=O)=O</smiles>

92

potential applications and tentative mechanisms is presented in Table 2.

\section{Anticancer Drugs and Bioactive Compounds}

Cancer is considered a major challenge to public health. There are many pyridine-containing drugs in the FDA database (Figure 52), eg, axitinib - a tyrosine kinase inhibitor - developed by Pfizer. For the treatment of cancer, other kinase inhibitors containing the pyridinering system are shown in Figure 53.
Figure 49 Pyroxicam binds with water-channel AQP4 to prevent cerebral ischemia.<smiles>CCOC(=O)C1=C(C)NC(C)=C(C(=O)OCC)C1C(=O)NCCS(=O)(=O)[O-]</smiles>

91

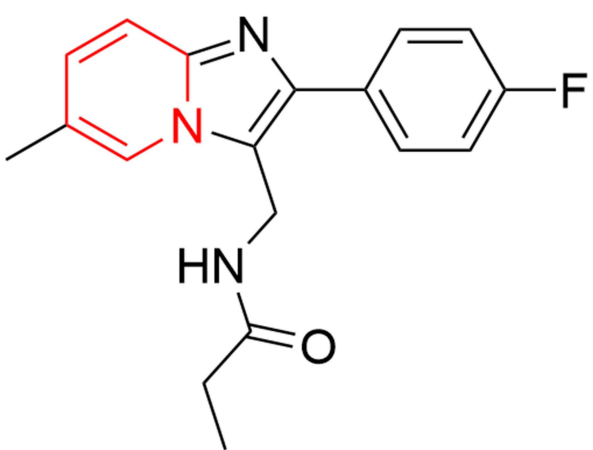

93
Figure $\mathbf{5 0}$ Neuroprotective agent.

Recently, the effectiveness of chemotherapeutic agents has been severely limited by tumor resistance. ${ }^{238,239}$ In a very recent study, pyridine-thiazole hybrid compounds were studied by Alqahtani et al. These hybrids contained (hydrazonomethyl)phenoxy-acetamide spacers, and novel compounds were evaluated for their cytotoxicity potential against normal fibroblast cells (WI38), breast cancer (MCF7), laryngeal carcinoma (Hep2), prostate cancer (PC3), and liver carcinoma (HepG2). The drug 5-fluorouracil $(5-\mathrm{Fu})$ was employed as the standard during these experiments. Promising anticancer activity against the HepG2 and MCF7 cell lines was reported for compounds 
Table 2 Summary of neurogenic/neuroprotective compounds with pyridine or dihydropyridine scaffolds

\begin{tabular}{|c|c|c|c|}
\hline $\begin{array}{l}\text { Compound } \\
\text { ID }\end{array}$ & Scaffold type & $\begin{array}{l}\text { Potential } \\
\text { application }\end{array}$ & Tentative mechanism \\
\hline 86 & Pyridine & Alzheimer's & AChE inhibition \\
\hline 87 & $\begin{array}{l}\text { Pyridine and } \\
\text { dihydropyridine }\end{array}$ & Alzheimer's & $\begin{array}{l}\text { Increase expression of GAD67 enzyme in hippocampus converts glutamate } \\
\text { to GABA }\end{array}$ \\
\hline 88 & Pyridine & Parkinson's & ND \\
\hline 89 & Pyridine & Parkinson's & ND \\
\hline 90 & DHP & Parkinson's & Enhances caspase $3^{+}$cells in the brain \\
\hline 91 & DHP & Parkinson's & Enhances caspase $3^{+}$cells in the brain \\
\hline 92 & Pyridine & Cerebral ischemia & Binds with water-channel AQP4 to prevent cerebral ischemia \\
\hline 93 & Pyridine & Schizophrenia & Potential affinity for serotonin $5 \mathrm{HT}_{2}$ and $5 \mathrm{HT}_{\mathrm{x}}$ receptors \\
\hline 94 & DHP & Alzheimer's & AChE inhibition \\
\hline 95 & Pyridine & Schizophrenia & AChE inhibition \\
\hline 96 & Pyridine & Schizophrenia & AChE inhibition \\
\hline 97 & Pyridine & Schizophrenia & AChE inhibition \\
\hline 98 & Pyridine & Schizophrenia & AChE inhibition \\
\hline 99 & Pyridine & Schizophrenia & AChE inhibition \\
\hline
\end{tabular}

Abbreviation: ND, not determined.

100 and 101, with $\mathrm{IC}_{50}$ values of 5.36 and $8.76 \mu \mathrm{M}$, respectively (Figure 54). Interestingly, both compounds had weak cytotoxic effects on normal cell lines (WI38). Docking analysis revealed valuable information about the binding sites, wherein the synthesized compounds interacted with ROCK1 protein-kinase cavity. It can be safely assumed that combining pyridine and thiazole moieties in one molecular platform via a phenoxyacetamide spacer potentially results in novel compounds with considerable synergistic anticancer effects. ${ }^{240}$

Schiff-based pyridine derivatives containing 4-thiazolidinones and azetidin-2-ones bearing pyrazolo[3,4- $b]$ pyridine moiety have also been prepared. Their antiproliferative activity was tested using sulforhodamine B assays. In hepatocellular carcinoma (HB8065) cells, the compounds exhibited remarkable cytotoxic effects. Among the compounds assayed, 102-106 had exceptionally high antiproliferative activity $\left(\mathrm{IC}_{50}\right.$ $=0.0091-0.0211 \mu \mathrm{M}$ ) against breast carcinoma cells (MCF7), whereas the standard drug doxorubicin had an $\mathrm{IC}_{50}$ of 0.099 $\mu \mathrm{M}$. Compound 102 displayed significantly high antiproliferative effects against MCF7 and $\mathrm{HB} 8065$, with $\mathrm{IC}_{50}$ of 0.0211 $\mu \mathrm{M}$ and $1.65 \mu \mathrm{M}$, respectively. These findings imply that these compounds (Figure 55) are highly promising leads in the pursuit of new antiproliferative agents. ${ }^{188}$

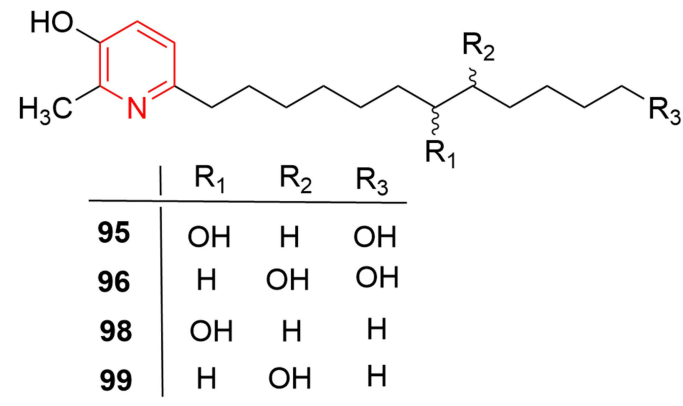<smiles>COC(=O)CCCc1ccc(O)c(C)n1</smiles>

97

Figure 5 I Neurogenically active pyridine alkaloids isolated from Senna and Cassia spp. 


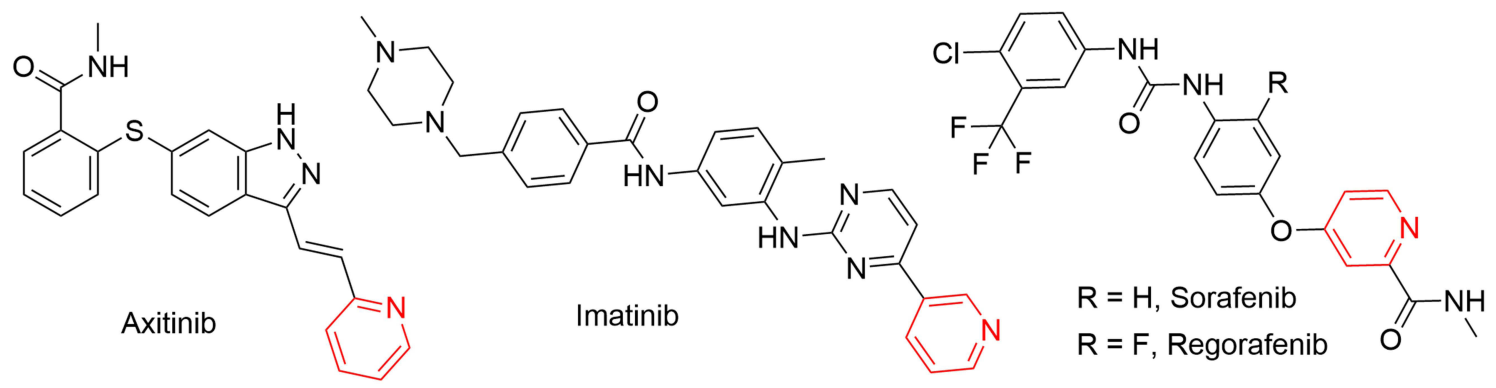

Figure 52 Pyridine-containing anticancer drugs in FDA database.

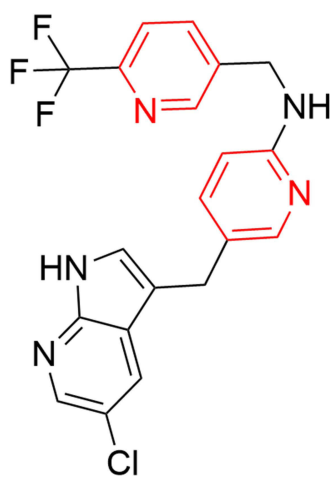

Pexidartinib

$\left(\mathrm{IC}_{50}=20 \mathrm{nM}\right)$

Daiichi Sankyo

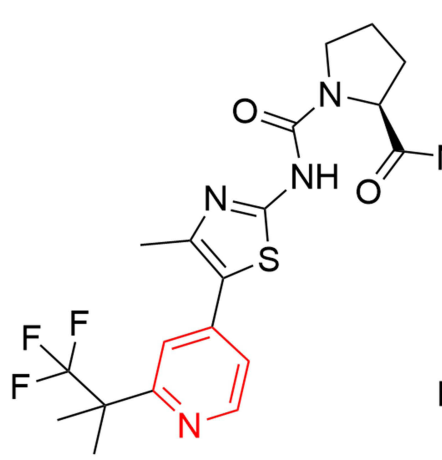

Alpelisib

$\left(\mathrm{IC}_{50}=5 \mathrm{nM}\right)$

Novartis Pharmaceuticals

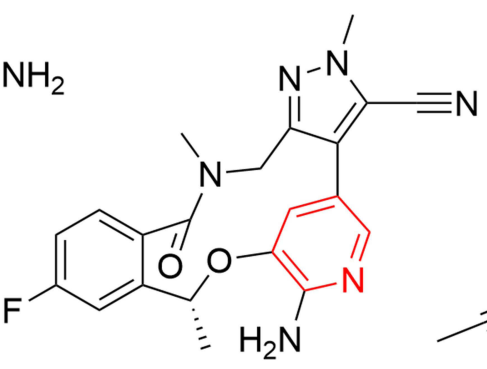

Lorlatinib $\left(\mathrm{IC}_{50}=10 \mathrm{nM}\right)$

Pfizer Inc.<smiles>CC#CC(=O)N1CCC[C@H]1c1nc(-c2ccc(C(=O)Nc3ccccn3)cc2)c2c(N)nccn12</smiles>

Acalabrutinib

$\left(\mathrm{IC}_{50}=3 \mathrm{nM}\right)$

AstraZeneca<smiles>CCN1CCN(Cc2ccc(Nc3ncc(F)c(-c4cc(F)c5nc(C)n(C(C)C)c5c4)n3)nc2)CC1</smiles>

Abemaciclib

$\left(\mathrm{IC}_{50}=2 \mathrm{nM}\right)$

Eli Lilly Inc.<smiles>CCOc1cc2ncc(C#N)c(Nc3ccc(OCc4ccccn4)c(Cl)c3)c2cc1NC(=O)/C=C/CN(C)C</smiles>

Neratinib

$\left(\mathrm{IC}_{50}=92 \mathrm{nM}\right)$

Puma Biotechnology Inc.

Figure 53 FDA-approved kinase inhibitors with pyridine scaffolds.

Enasidenib and ivosidenib (Figure 56) were recently approved by the FDA for leukemia. ${ }^{241}$ Both are pyridinecontaining first-in-class drugs.

In 2015, Sailaja et al studied pyridine-indole hybrids and had promising cytotoxicity results, ${ }^{242}$ wherein compounds 107-109 had promising activity against K562 leukemia cells (Figure 57).

Viradiya et al synthesized a series of benzylpyridinium-bearing dihydropyridines (Figure 58). In MTT assays, these compounds had excellent anticancer activity against colorectal adenocarcinoma Caco2, lung cancer A549, and glioblastoma U87MG cell lines. For these cell lines, compounds 110-113 showed better anticancer activity than the widely used drugs carboplatin, gemcitabine, and daunorubicin. Compound $\mathbf{1 1 2}$ was the most potent in the series, 3.6-fold as potent as carboplatin and 4.2-fold as active than gemcitabine. Tthe mechanisms of action showed that the tested compounds induced cell death via 
<smiles>CCOC(=O)C1SC(N/N=C/c2ccc(OCC(=O)Nc3ccccn3)cc2)=NC1=O</smiles>

100: $I C_{50}=5.36 \pm 0.23 \mu \mathrm{M}$

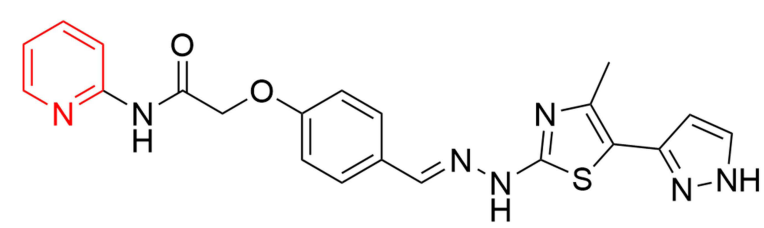

101: $I C_{50}=5.84 \pm 0.17 \mu \mathrm{M}$

Figure 54 Pyridine-thiazole hybrids with remarkable anticancer effect in MCF7 breast adenocarcinoma.<smiles>Cc1c(Br)c(C)n(C(=S)Nc2ccccc2)c(=O)c1C#N</smiles>

102<smiles>Cc1nc2[nH]nc(/C=C/c3ccc(Cl)cc3)c2c(C)c1Br</smiles>

103 $425.280 \pm 4.031$ (HB8065)<smiles>Cc1nc2[nH]nc(/C=C/c3c[nH]c4ccccc34)c2c(C)c1Br</smiles>

$0.0198 \pm 0.00015$ (MCF-7) $3.627 \pm 0.014$ (HB8065)

$\mathrm{IC}_{50}(\mu \mathrm{M})\left\{\begin{array}{l}0.0211 \pm 0.00003(\mathrm{MCF}-7) \\ 1.65 \pm 0.011(\mathrm{HB} 8065)\end{array}\right.$<smiles>Cc1nc2[nH]nc(N3C(=O)CSC3c3c[nH]c4ccccc34)c2c(C)c1Br</smiles>

105

$$
\mathrm{IC}_{50}(\mu \mathrm{M})\left\{\begin{array}{l}
2.421 \pm 0.012(\mathrm{MCF}-7) \\
680.89 \pm 5.357(\mathrm{HB} 8065)
\end{array}\right.
$$<smiles>Cc1nc2[nH]nc(NN=C(C#N)C#N)c2c(C)c1Br</smiles>

$0.0091 \pm 0.000016$ (MCF-7) $110.131 \pm 0.76$ (HB8065)

Figure 55 Pyrazolo[3,4-b] pyridine- and dihydropyridine-derived compounds.<smiles>CC(C)(O)CNc1nc(Nc2ccnc(C(F)(F)F)c2)nc(-c2cccc(C(F)(F)F)n2)n1</smiles><smiles>N#Cc1ccnc(N2C(=O)CC[C@H]2C(=O)N(c2cncc(F)c2)[C@H](C(=O)NC2CC(F)(F)C2)c2ccccc2Cl)c1</smiles>

Figure $\mathbf{5 6}$ Oncology drugs for leukemia recently approved by the FDA. 

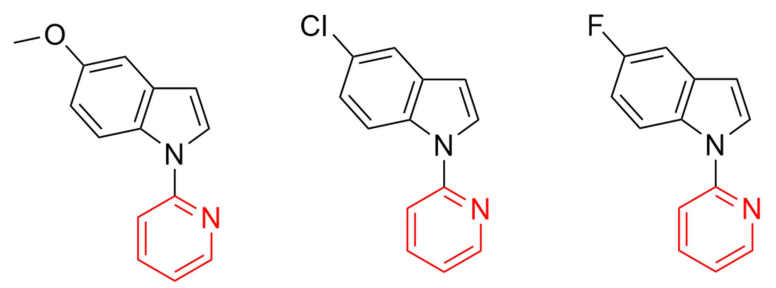

$107=68.8 \%$

$108=41.5 \%$

$109=39.9 \%$

\%age inhibition against K562 leukemia cells

Figure $\mathbf{5 7}$ Substituent effect on cytotoxicity by pyridine-indole hybrid compounds.<smiles></smiles>

110

$$
\begin{aligned}
& \frac{\mathrm{IC}_{50}(\mu \mathrm{M})}{\mathrm{A} 549=} 2.96 \pm 0.91 \\
\mathrm{Caco}-2= & 3.45 \pm 1.15 \\
\mathrm{U}-87 \mathrm{MG}= & 2.97 \pm 0.47
\end{aligned}
$$

111

$\frac{I C_{50}(\mu \mathrm{M})}{6.28 \pm 0.95}$
$1.85 \pm 0.97$
$2.56 \pm 0.52$

112

$\frac{I C_{50}(\mu \mathrm{M})}{2.03 \pm 0.74}$
$2.50 \pm 0.63$
$2.41 \pm 0.24$

113

$$
\begin{gathered}
I C_{50}(\mu \mathrm{M}) \\
\hline 2.09 \pm 0.72 \\
4.42 \pm 0.13 \\
2.63 \pm 0.61
\end{gathered}
$$

Figure 58 ।,4-Dihydropyridine-containing benzylpyridinium moieties with remarkable anticancer activity.<smiles>N=c1c2c(-c3ccccc3)c(C(=O)O)c(Nc3ccccc3)nc2ncn1N</smiles>

114<smiles>N#Cc1c(Nc2ccccc2)nc2[nH]c(=S)n(-c3ccccc3)c(=N)c2c1-c1ccccc1</smiles>

115<smiles>Clc1nnnc2nc(Nc3ccccc3)cc(-c3ccccc3)c12</smiles>

116

$I_{50}(\mu \mathrm{M})$
$5.36 \pm 0.6$
$6.73 \pm 0.5$

Figure 59 Fused heterocyclic derivatives containing pyridine moieties. 

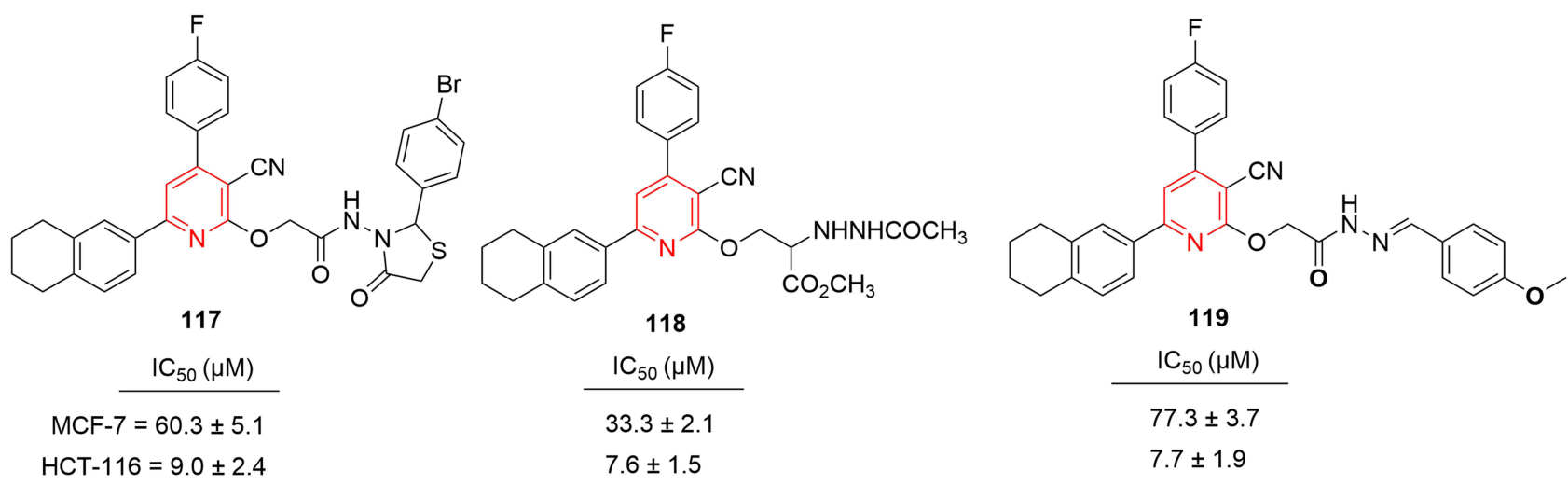

Figure 60 Tetralin-pyridine hybrids.

apoptosis. The excellent anticancer capabilities of benzylpyridinium-bearing dihydropyridines might be helpful against MDR cancer strains. However, to gain better knowledge of their mode of action, further in-depth mechanistic validation is still needed for these types of compounds. $^{243}$

Naglaa et al recently designed a series of pyridinecontaining anticancer agents (Figure 59). The newly synthesized compounds were investigated for in vitro growth activity against mammary-gland carcinoma (MCF7) and human hepatocellular carcinoma (HepG2) cell lines. Doxorubicin, an anticancer drug, was employed as a comparative standard under the same conditions. Against HepG2 and MCF7, most of the newly synthesized compounds showed significantly potent anticancer activity. The derivatives 114-116 displayed remarkable activity. To further confirm the hypothesized mechanism, molecular docking studies were conducted to evaluate affinity between the compounds and their binding energy with the enzyme. For potent compounds, the calculated binding energies were in good agreement with their activity against the MCF7 and HepG2 cell lines. ${ }^{244}$

Recently, Eman et al reported an important contribution toward new anticancer agents by developing a series of tetralin-pyridine hybrids, starting from 2-(pyridin-2-yl [oxy])acetohydrazide in appreciable yields (Figure 60). MTT assays were employed to evaluate the cytotoxic activity of these compounds against human MCF7 and HCT116 cells. $\mathrm{IC}_{50}$ values were 7.7-9.0 $\mu \mathrm{M}$ against HCT116 cancer cells, comparable to the standard drug doxorubicin $\left(\mathrm{IC}_{50}=\right.$ $8 \mu \mathrm{M})$. The derivative 117-119 showed $\mathrm{IC}_{50}$ values of 21.0, 33.3, and $60.3 \mu \mathrm{M}$, respectively, against MCF7 cells. It can tentatively be assumed that a tetralin-pyridine backbone is an effective antitumor pharmacophoric moiety against MCF7 cells. These findings suggest that all the tested compounds are more active against human colon cancer cells than human breast cancer cells. ${ }^{245}$

Phosphodiesterases (PDEs) have been recognized as important targets in cancer therapy, due to their critical role in apoptosis induction and inhibition of tumor-cell

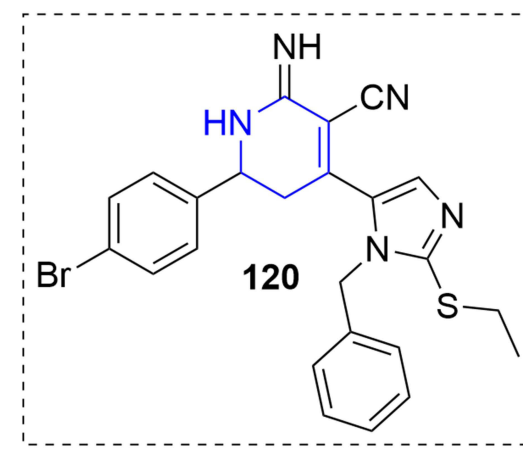

\author{
$\mathrm{IC}_{50}$ \\ Hela $=34.3 \pm 2.6 \mu \mathrm{M}$ \\ MCF-7 $=50.18 \pm 1.11 \mu \mathrm{M}\}$ Growth inhibition \\ $3.76 \pm 1.03 \mathrm{nM}$ PDE3 inhibition
}

Figure 6I Highly potent anticancer compound with PDE3-inhibitory effect. 


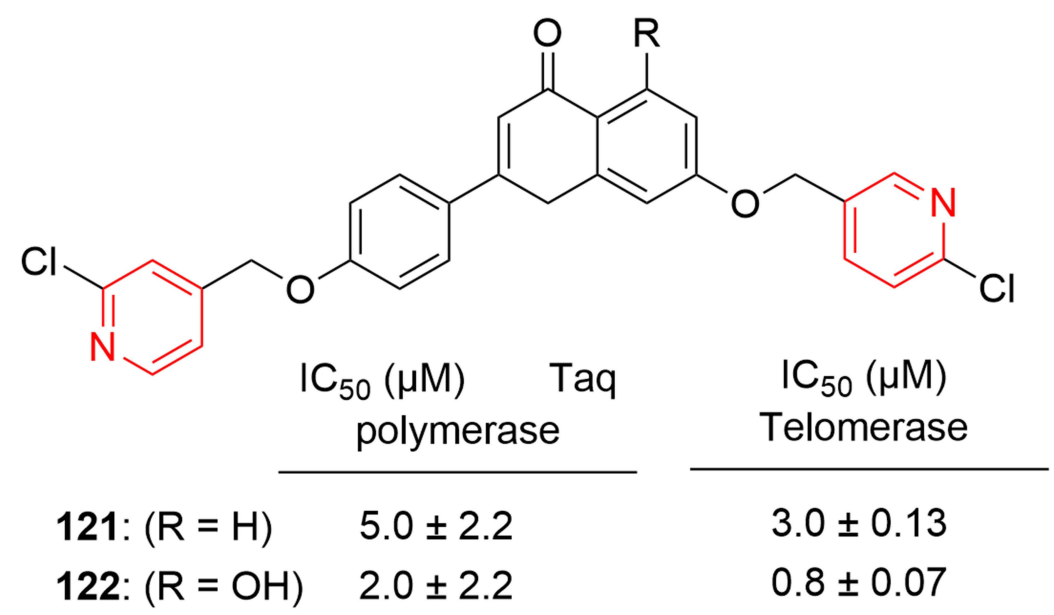

Figure 62 Antitumor agents with telomerase-inhibitory effects.

growth. Some nonselective PDE inhibitors, eg, aminophylline and theophylline, have been recognized as growth regulators in various carcinoma cell lines, suggesting a potential role as anticancer drugs for PDE inhibition. A range of imidazoaryl-containing dihydropyridine compounds and their 2-oxo isostere derivatives was synthesized and evaluated as PDE inhibitors by Atieh et al. The cytotoxic effect was also checked for the HeLa and MCF7 cell lines. An extraordinarily high PDE3A inhibitory effect was demonstrated by compound $\mathbf{1 2 0}$, with $\mathrm{IC}_{50}$ of 3.76 $\pm 1.03 \mathrm{nM}$ (Figure 61). Compound 89 also displayed significantly high cytotoxicity effects against MCF7 and HeLa cells $\left(\mathrm{IC}_{50} 50.18 \pm 1.11\right.$ and $34.3 \pm 2.6 \mu \mathrm{M}$, respectively). The strong association between $\mathrm{IC}_{50}$ values of cytotoxicity and PDE3A inhibition support the notion that PDE3 inhibitors could be used as cytotoxic entities. According to SAR investigations and docking studies, hydrophobic interactions were found to be equally important in the formation of hydrogen bonds intended for PDE3 inhibition and cytotoxic effects of proposed derivatives. $^{246}$

The crucial role of telomerase in tumor growth makes it a promising target for cancer treatment and other age- related illnesses. Telomere and telomerase are known to be linked to the progression of gastric cancer. Xin-Hua and others synthesized flavone containing 2-chloro-pyridine derivatives for telomerase inhibition (Figure 62). Modified telomeric repeat-amplification protocol assays were used to evaluated the telomerase-inhibitory effect of the compounds, and $\mathbf{1 2 1}$ and $\mathbf{1 2 2}$ showed significant activity against the SGC7901 gastric cancer cell line, with $\mathrm{IC}_{50}$ of $18.45 \pm 2.79 \mu \mathrm{g} / \mathrm{mL}$ and $22.28 \pm 6.26 \mu \mathrm{g} / \mathrm{mL}$, respectively. In order to determine the probable binding mode, dockingsimulation studies were performed at 3DU6-active sites. Compound 122 was a more effective inhibitor of telomerase by binding with the telomerase-active site. ${ }^{247}$

Fatma et al also reported disubstituted pyridines (Figure 63) and studied their anticancer activity against the HepG2 cells. Compounds 123-125 were found to have promising activity comparable to standard drugs and 5fluorouracil. ${ }^{248}$

In 2016, pyridine-pyrimidine hybrid ring system-containing compounds were screened at $10 \mu \mathrm{M}$ against various cancer-cell lines, ${ }^{249}$ ande compound 126 (Figure 64) showed promising inhibitory effects against the NCI60 cell lines, with $\mathrm{IC}_{50}$ of $1.40 \mu \mathrm{M}$ for $\mathrm{UO} 31,1.55 \mu \mathrm{M}$ for

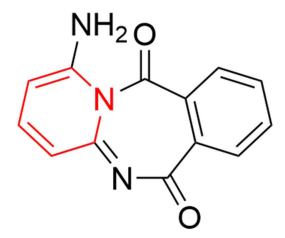

123: $I C_{50}=3.36 \mu \mathrm{g} / \mathrm{ml}$

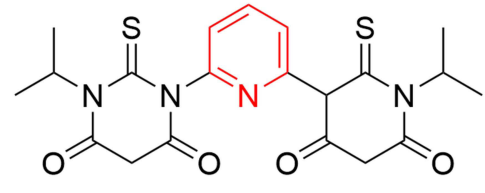

124: $\mathrm{IC}_{50}=3.09 \mu \mathrm{g} / \mathrm{ml}$

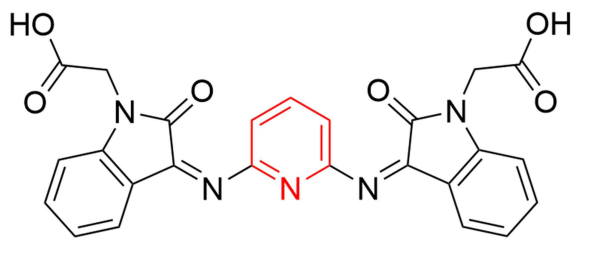

125: $\mathrm{IC}_{50}=2.08 \mu \mathrm{g} / \mathrm{ml}$

Figure 63 Compounds with remarkable activity against HepG2 liver cancer cells. 


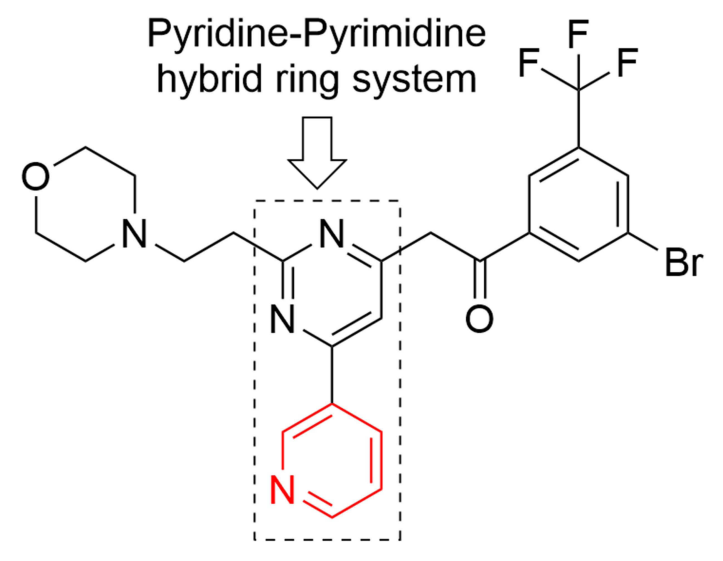

126

Figure 64 Pyridine-pyrimidine hybrid ring system containing compound I 26 with inhibitory effects against $\mathrm{NCl} 60$ cell lines.

SNB75, $1.60 \mu \mathrm{M}$ for M14, 1.62 $\mu \mathrm{M}$ for SKMEL5 and 1.77 $\mu \mathrm{M}$ for Colo205 cells.

Süss-Fink et al reported highly potent pyridine-based compounds [14] and evaluated their anticancer potential in
A2780 (ovarian cancer) and A2780cisR (cisplatin-resistant cancer) cells. Pyridine-4-carboxylate containing a lipophilic chain of ten carbon atoms $\mathbf{1 2 7}$ was highly cytotoxic, with $\mathrm{IC}_{50}$ values of $5 \mu \mathrm{M}$ and $11 \mu \mathrm{M}$ for A2780 and A2780cisR cell lines, respectively (Figure 65). Surprisingly, the arene ruthenium complex of $\mathbf{1 2 7}$ had remarkably high anticancer activity against both lines, the $\mathrm{IC}_{50}(2 \mu \mathrm{M}$ for A2780) of $\mathbf{1 2 8}$ being fivefold that of $127 .{ }^{250}$

Interestingly, introduction of an $\mathrm{OH}$ group to $\mathbf{1 2 7}$ renders the new compound 129, which is almost inactive $\left(\mathrm{IC}_{50}=162 \mu \mathrm{M}\right.$ for $\mathrm{A} 2780$ and $\mathrm{IC}_{50}=208 \mu \mathrm{M}$ for A2780cisR; Figure 66). However, the $p$-cymene ruthenium complex 130 shows very high anticancer activity in the submicromolar range, with $\mathrm{IC}_{50}$ of $0.18 \mu \mathrm{M}$. $^{251}$

Pyridine hybrids of isatin have been found to demonstrate antiproliferative effects in MCF7, HT29, and HepG2 cells, wherein compounds 131-133 had noteworthy activity (Figure 67). ${ }^{252}$

Higher activity in MCF7, U87MG, and HCT116 cells have recently been studied with $[1,2,4]$ triazolo

Decyl group was the optimal length of lipophilic chain for the best cytotoxicity results.

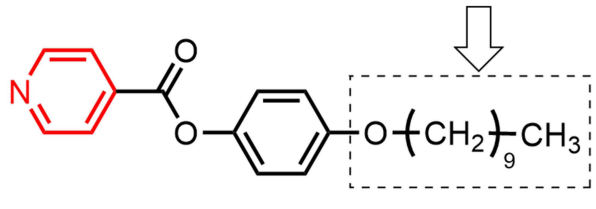

127

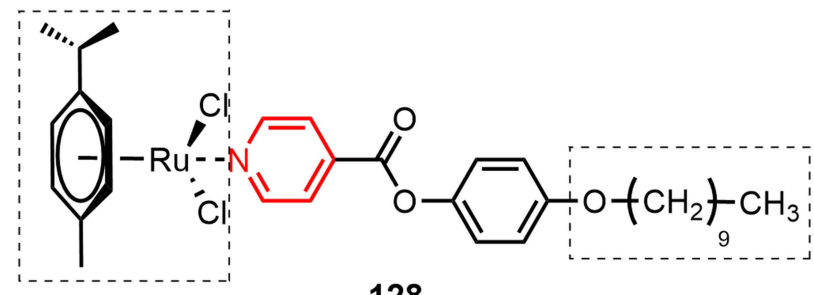

128

Arene ruthenium moiety further enhanced the cytotoxicity by 5 -folds

Figure 65 Isonicotinic ester containing compounds 127 and 128

Introduction of hydrophilic -OH group on decyl chain made the compound non-cytotoxic

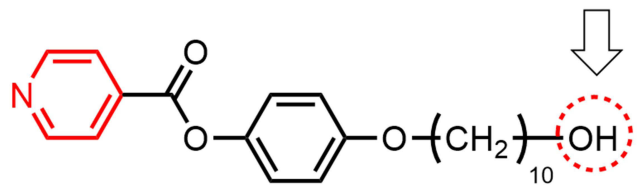

129

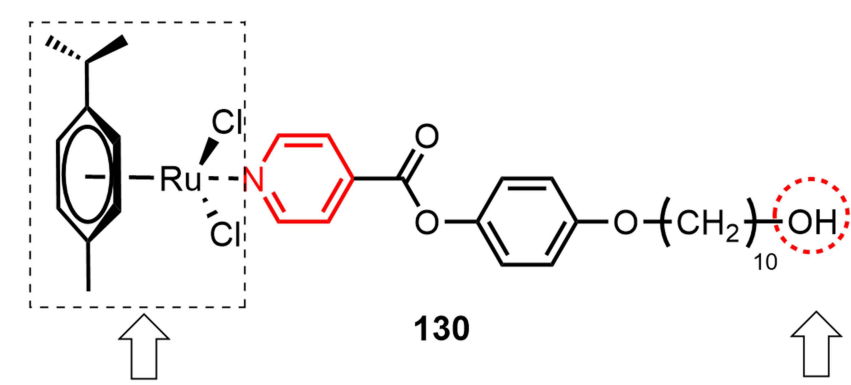

Arene ruthenium moiety in combination with hydrophilic -OH group on decyl chain made this compound highly cytotoxic in sub micromolar range.

Figure 66 p-cymene-ruthenium complex 130 with submicromolar anticancer activity against ovarian cancer cell lines. 
<smiles>Cc1nc(-c2ccccc2)ccc1C(=O)/C=C1\C(=O)Nc2ccccc21</smiles>

131<smiles>Cc1cc(-c2ccccc2)cnc1C(=O)N/N=C1\C(=O)Nc2ccccc21</smiles>

$I_{50}(\mu \mathrm{M})\left\{\begin{array}{l}\mathrm{HepG}_{2}=2.5 \pm 0.39 \\ \mathrm{~A} 549=19.3 \pm 1.34 \\ \text { MCF-7 }=11.6 \pm 0.93\end{array}\right.$

$23.5 \pm 1.57$

$27.6 \pm 1.86$

$71 \pm 3.58$
$8.7 \pm 0.91$

$10.8 \pm 1.15$

$6.3 \pm 0.79$

Figure 67 Structure simplification in pyridine-isatin hybrids resulted in better $I C_{50}$ values.<smiles>COc1ncc(-c2ccc3nc(NC(C)=O)nn3c2)cc1NS(=O)(=O)c1ccc(F)cc1</smiles>

134

Figure $68[1,2,4]$ Triazolo[I,5-a]pyridinylpyridine-containing highly potent anticancer agent.

\section{[1,5-a]pyridinylpyridines. ${ }^{253}$ Compound $\mathbf{1 3 4}$ had} remarkably high anticancer effects in these cell lines (Figure 68).

In diphenyl-1-(pyridin-3-yl)ethylphosphonates, ${ }^{254}$ the compounds 135 and 136 also demonstrated cytotoxic effect against MCF7 and HepG2 cells (Figure 69).

Pyridine and dihydropyridine are considered attractive scaffolds for anticancer drug development, since many drugs containing these moieties are already in the market, having shown remarkable results. Rational design of new anticancer drugs can be achieved by incorporating these scaffolds into the backbone of bioactive molecules, followed by their analysis with computational methods to predict highly potent<smiles>Cc1ccc(NC(C)(c2cccnc2)P(=O)(Oc2ccccc2)Oc2ccccc2)cc1</smiles>

135

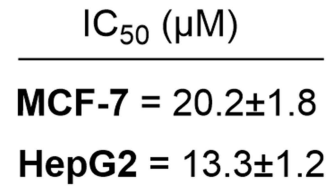<smiles>CC(Nc1ccc(Cl)cc1)(c1cccnc1)P(=O)(Oc1ccccc1)Oc1ccccc1</smiles>

136

$$
\begin{gathered}
\mathrm{IC}_{50}(\mu \mathrm{M}) \\
\hline \text { MCF-7 }=17.6 \pm 1.8 \\
\text { HepG2 }=15.8 \pm 1.6
\end{gathered}
$$

Figure 69 Diphenyl I-(pyridin-3-yl)ethylphosphonate-containing anticancer agents.

candidates. Drug repurposing of existing pyridine- and dihydropyridine-containing pharmaceuticals should also be explored to accelerate the discovery of new anticancer drugs.

\section{Conclusion}

The present review is a critical analysis of various drugs and research on the design and development of assorted derivatives of pyridine- and dihydropyridine-based compounds. They have been characterized on the basis of their pharmacological activity. Specific structural features pertinent to particular activity have also been discussed. The pyridine core has far greater tractability to produce anti-infectious and anticancer agents. This is evident from the fact that the FDA has recently 
approved many pyridine-containing antibiotics, such as ceftaroline fosamil (2010), tedizolid (2014), ceftazidime (2015), and delafloxacin (2017). In the database, one can also find isoniazid, ethionamide, and prothionamide, which are highly effective against mycobacteria for the treatment of tuberculosis. Combination of pyridine scaffolds with oxazolidinone hold great promise in this regard, since many such compounds have recently appeared in contemporary literature with remarkable antibacterial effects. For example, the compounds 23-25 have potent activity against a wide range of drug-resistant bacteria, as well as M. catarrhoides and H. influenzae, both in in vitro and in vivo evaluations. The FDA database also contains many pyridine-containing antiviral drugs, such as nevirapine, tipranavir, doravirine, and indinavir, which are being employed to manage HIV infection. During the last 10 years, many isothiazolopyridine-based compounds, such as 61-66, were developed as selective GAK inhibitors to thwart the initial and latestage viral life cycle. Pyridine-containing oxicam compounds were also found to be promising against musculoskeletal disorders, such as osteoarthritis and rheumatoid arthritis. For the treatment of cancer, pyridine was the integral part of numerous FDA-approved kinase inhibitors, such as acalabrutinib, neratinib, abemaciclib, alpelisib, lorlatinib, and pexidartinib, whereas many pyrazolo[3,4-b]pyridine-containing compounds (102-106), ha exceptionally high antiproliferative activity $\left(\mathrm{IC}_{50}=0.0091-0.0211 \mu \mathrm{M}\right)$ against MCF7 cells, while the standard drug doxorubicin had an $\mathrm{IC}_{50}$ of 0.099 $\mu \mathrm{M}$. In arene-ruthenium complexes with pyridine scaffolds, Süss-Fink et al found highly potent anticancer effects, with $\mathrm{IC}_{50}$ values being in the submicromolar range. Pyridine-containing ruthenium compounds hold great promise for the replacement of cisplatin-based anticancer drugs. Dihydropyridine ring-containing drugs mostly act as calcium-channel blockers and ware frequently employed for the treatment of hypertension and heart-related problems. Such drugs include nimodipine, ciclopirox, efonidipine, nifedipine, milrinone, and amrinone. Cholesterol-lowering compounds (18-22) containing dihydropyridine rings were developed due to different antidyslipidemic and antioxidant effects of such a scaffold. Dihydropyridine-containing 80-82 ware able to inhibit $11 \beta$ HSD1 for the potential cure of diabetes mellitus. In the literature, one can also find numerous pyridine- or dihydropyridinecontaining compounds (86-94) for the potential treatment of neurodegenerative disorders, along with many drugs-repurposing examples, such as dolutegravir, mastinib, nilvadipine, nilotinib, clioquinol, imatinibs. Despite years of research, further work is still warranted to optimize their effects and understand their mechanisms of action. In summary, pyridine- and dihydropyridine-containing compounds combined with broadened chemical space will help medicinal chemists to design bioactive molecules for specific targets. Briefly, in view of the colossal structural diversity of pyridine- and dihydropyridinecontaining compounds, the existing literature barely scratches the surface of possibilities for their pharmacological application. Therefore, the interest in them is unlikely to die out anytime soon. We will see an increase in the structure, application, and diversity of pyridine- and dihydropyridine-containing compounds, with great potential for new cardiovascular, antiinflammatory, anti-infectious, neurogenic, and anticancer drugs containing the two heterocycles in the forthcoming decade.

\section{Abbreviations}

AChE, acetylcholinesterase; COX, cyclooxygenase; DHP, dihydropyridine; FDA, Food and Drug Administration; GABA, $\gamma$-aminobutyric acid; GAK, G-associated kinase; HepG2, Hepatocellular carcinoma; MDR, multidrug-resistant; MIC, minimum inhibitory concentration; MRSA, methicillinresistant Staphylococcus aureus; NAD, nicotinamide adenine dinucleotide; NADP, NAD phosphate; NO, nitric oxide; NSAIDs, nonsteroidal anti-inflammatory drugs; PDE, phosphodiesterase; PL, phospholipid-lowering; ROS, reactive oxygen species.

\section{Acknowledgments}

The authors acknowledge grant GZYYGJ2020023 from the Sino-Pakistan Cooperation Center on Traditional Chinese Medicine, located at the International Center for Chemical and Biological Sciences, University of Karachi, Karachi, Pakistan. Thanks are also due to The Affiliated Hospital of Qingdao University, Qingdao, Shandong, China and HEJ Research Institute of Chemistry, International Center for Chemical and Biological Sciences, University of Karachi.

\section{Author Contributions}

All authors made substantial contributions to conception and design, acquisition of data, or analysis and interpretation of data, took part in drafting the article or revising it critically for important intellectual content, agreed to submit to the current journal, gave final approval to the version to be published, and agree to be accountable for all aspects of the work.

\section{Disclosure}

The authors declare no conflicts of interest in this work. 


\section{References}

1. Wang S, Yuan XH, Wang SQ, Zhao W, Chen XB, Yu B. FDAapproved pyrimidine-fused bicyclic heterocycles for cancer therapy: synthesis and clinical application. Eur $J$ Med Chem. 2021;214:113218. doi:10.1016/j.ejmech.2021.113218

2. Bull JA, Mousseau JJ, Pelletier G, Charette AB. Synthesis of pyridine and dihydropyridine derivatives by regio- and stereoselective addition to N-activated pyridines. Chem Rev. 2012;112 (5):2642-2713. doi:10.1021/cr200251d

3. Boström J, Brown DG, Young RJ, Keserü GM. Expanding the medicinal chemistry synthetic toolbox. Nat Rev Drug Discov. 2018;17(10):709-727. doi:10.1038/nrd.2018.116

4. Wang L, Bharti KR, Pavlov PF, Winblad B. Small molecule therapeutics for tauopathy in Alzheimer's disease: walking on the path of most resistance. Eur J Med Chem. 2021;209:112915. doi:10.1016/j. ejmech.2020.112915

5. Jubete G, Puig de la Bellacasa R, Estrada-Tejedor R, Teixidó J, Borrell JI. Pyrido[2,3-d]pyrimidin-7(8H)-ones: synthesis and biomedical applications. Molecules. 2019;24(22):4161. doi:10.3390/ molecules 24224161

6. Mammoliti O, Palisse A, Joannesse C, et al. Discovery of the S1P2 antagonist GLPG2938 (1-[2-Ethoxy-6-(trifluoromethyl)-4-pyridyl]-3[[5-methyl-6-[1-methyl-3-(trifluoromethyl)pyrazol-4-yl]pyridazin-3yl]methyl]urea), a preclinical candidate for the treatment of idiopathic pulmonary fibrosis. J Med Chem. 2021;64(9):6037-6058. doi:10.1021/ acs.jmedchem. $1 \mathrm{c} 00138$

7. Ran K, Zeng J, Wan G, et al. Design, synthesis and biological evaluations of a series of Pyrido[1,2-a]pyrimidinone derivatives as novel selective FGFR inhibitors. Eur $J$ Med Chem. 2021;220:113499. doi:10.1016/j.ejmech.2021.113499

8. Yerragunta V, Patil P, Anusha V, Kumaraswamy T, Suman D, Samhitha T. Pyrimidine and its biological activity: a review. PharmaTutor. 2013;1(2):39-44.

9. Desai NC, Somani H, Trivedi A, et al. Synthesis, biological evaluation and molecular docking study of some novel indole and pyridine based 1,3,4-oxadiazole derivatives as potential antitubercular agents. Bioorg Med Chem Lett. 2016;26(7):1776-1783. doi:10.1016/j.bmcl.2016.02.043

10. Comins DL, Higuchi K, Young DW. Dihydropyridine preparation and application in the synthesis of pyridine derivatives. $A d v$ Heterocycl Chem. 2013;110:175-235. doi:10.1016/B978-0-12408100-0.00006-9

11. Lin SX, Curtis MA, Sperry J. Pyridine alkaloids with activity in the central nervous system. Bioorg Med Chem. 2020;28 (24):115820. doi:10.1016/j.bmc.2020.115820

12. Pollak N, Dölle C, Ziegler M. The power to reduce: pyridine nucleotides - small molecules with a multitude of functions. Biochem J. 2007;402(2):205-218. doi:10.1042/BJ20061638

13. Coffinier D, Kaim EL, Grimaud L, Ruijter E, Orru RVA. A new multicomponent reaction for the synthesis of pyridines via cycloaddition of azadienes and ketenimines. Tetrahedron Lett. 2011;52(23):3023-3025. doi:10.1016/j.tetlet.2011.04.007

14. Zakharychev VV, Kuzenkov AV, Martsynkevich AM. Good pyridine hunting: a biomimic compound, a modifier and a unique pharmacophore in agrochemicals. Chem Heterocycl Compd. 2020;56(12):1491-1516. doi:10.1007/s10593-020-02843-w

15. Neely JM, Rovis T. Pyridine synthesis by $[4+2]$ cycloadditions of 1-azadienes: hetero-Diels Alder and transition metal-catalysed approaches. Org Chem Front. 2014;1(8):1010-1015. doi:10.1039/ C4QO00187G

16. Wan JP, Liu Y. Recent advances in new multicomponent synthesis of structurally diversified 1,4-dihydropyridines. RSC Adv. 2012;2 (26):9763. doi:10.1039/c2ra21406g
17. Alizadeh SR, Ebrahimzadeh MA. Antiviral activities of pyridine fused and pyridine containing heterocycles, a review (from 2000 to 2020). Mini Rev Med Chem. 2021;21. doi:10.2174/138955 7521666210126143558.

18. Villamizar-Mogotocoro A-F, Vargas-Méndez LY, Kouznetsov VV. Pyridine and quinoline molecules as crucial protagonists in the never-stopping discovery of new agents against tuberculosis. Eur J Pharm Sci. 2020;151:105374. doi:10.1016/j. ejps.2020.105374

19. Ioan P, Carosati E, Micucci M, et al. 1,4-Dihydropyridine scaffold in medicinal chemistry, the story so far and perspectives (Part 1): action in ion channels and GPCRs. Curr Med Chem. 2011;18 (32):4901-4922. doi:10.2174/092986711797535173

20. Velena A, Zarkovic N, Klusa V, et al. 1,4-dihydropyridines as tools for mitochondrial medicine against oxidative stress and associated metabolic disorders. Curr Org Chem. 2017;21(20). doi:10.2174/1385272821666170207104206

21. Prachayasittikul S, Pingaew R, Worachartcheewan A, et al. Roles of pyridine and pyrimidine derivatives as privileged scaffolds in anticancer agents. Mini Rev Med Chem. 2017;17(10):869-901. doi:10.2174/1389557516666160923125801

22. Álvarez R, Aramburu L, Puebla P, et al. Pyridine based antitumour compounds acting at the colchicine site. Curr Med Chem. 2016;23 (11):1100-1130. doi:10.2174/092986732311160420104823

23. Klusa V. Atypical 1,4-dihydropyridine derivatives, an approach to neuroprotection and memory enhancement. Pharmacol Res. 2016;113:754-759. doi:10.1016/j.phrs.2016.05.017

24. Peters J, Booth A, Peters R. Potential for specific dihydropyridine calcium channel blockers to have a positive impact on cognitive function in humans: a systematic review. Ther Adv Chronic Dis. 2015;6(4):160-169. doi:10.1177/2040622315582353

25. Lapidot I, Albeck A, Gellerman G, Shatzmiller S, Grynszpan F. 1,4-dihydropyridine cationic peptidomimetics with antibacterial activity. Int J Pept Res Ther. 2015;21(3):243-247. doi:10.1007/ s10989-015-9460-1

26. Bucci C, Mamdani MM, Juurlink DN, Tu JV. Dihydropyridine calcium channel blockers and cardiovascular outcomes in elderly patients: a population-based study. Can J Cardiol. 2008;24 (8):629-632. doi:10.1016/s0828-282x(08)70651-2

27. Bruncko M. Dihydropyridine-based calcium channel blockers for the treatment of angina pectoris and hypertension. In: Bioactive Heterocyclic Compound Classes. Weinheim, Germany: WileyVCH Verlag GmbH \& Co. KGaA; 2013:135-151. doi:10.1002/ 9783527664450.ch9

28. Ishii N, Matsumura T, Shimoda S, Araki E. Anti-atherosclerotic potential of dihydropyridine calcium channel blockers. $J$ Atheroscler Thromb. 2012;19(8):693-704. doi:10.5551/ jat. 12450

29. Mishra AP, Bajpai A, Rai AK. 1,4-dihydropyridine: a dependable heterocyclic ring with the promising and the most anticipable therapeutic effects. Mini Rev Med Chem. 2019;19(15):12191254. doi:10.2174/1389557519666190425184749

30. Khedkar SA, Auti PB. 1, 4-Dihydropyridines: a class of pharmacologically important molecules. Mini Rev Med Chem. 2014;14 (3):282-290. doi:10.2174/1389557513666131119204126

31. Edraki N, Mehdipour AR, Khoshneviszadeh M, Miri R. Dihydropyridines: evaluation of their current and future pharmacological applications. Drug Discov Today. 2009;14(2122):1058-1066. doi:10.1016/j.drudis.2009.08.004

32. Vitaku E, Smith DT, Njardarson JT. Analysis of the structural diversity, substitution patterns, and frequency of nitrogen heterocycles among U.S. FDA approved pharmaceuticals. $J$ Med Chem. 2014;57(24):10257-10274. doi:10.1021/ jm501100b 
33. Pennington LD, Moustakas DT. The necessary nitrogen atom: a versatile high-impact design element for multiparameter optimization. J Med Chem. 2017;60(9):3552-3579. doi:10.1021/acs. jmedchem.6b01807

34. Vanotti E, Amici R, Bargiotti A, et al. Cdc7 kinase inhibitors: pyrrolopyridinones as potential antitumor agents. 1. Synthesis and structure-activity relationships. J Med Chem. 2008;51(3):487501. doi:10.1021/jm700956r

35. Zheng X, Bauer P, Baumeister T, et al. Structure-based identification of ureas as novel nicotinamide phosphoribosyltransferase (Nampt) inhibitors. $J$ Med Chem. 2013;56(12):4921-4937. doi:10.1021/jm400186h

36. Hong SP, Liu KG, Ma G, et al. Tricyclic thiazolopyrazole derivatives as metabotropic glutamate receptor 4 positive allosteric modulators. J Med Chem. 2011;54(14):5070-5081. doi:10.1021/ jm $200290 \mathrm{z}$

37. Huang H, Degnan AP, Balakrishnan A, et al. Oxazolidinone-based allosteric modulators of mGluR5: defining molecular switches to create a pharmacological tool box. Bioorg Med Chem Lett. 2016;26 (17):4165-4169. doi:10.1016/j.bmcl.2016.07.065

38. Castellan P, Marchioni M, Castellucci R, et al. Abiraterone acetate for early stage metastatic prostate cancer: patient selection and special considerations. Ther Clin Risk Manag. 2018;14:23412347. doi:10.2147/TCRM.S159824

39. Basco L, Gillotin C, Gimenez F, Farinotti R, Bras J. In vitro activity of the enantiomers of mefloquine, halofantrine and enpiroline against Plasmodium falciparum. $\mathrm{Br} J \mathrm{Clin}$ Pharmacol. 1992;33(5):517-520. doi:10.1111/j.1365-2125.1992. tb04081.x

40. Raghuramulu N, Srikantia S, Rao B, Gopalan C. Nicotinamide nucleotides in the erythrocytes of patients suffering from pellagra. Biochem J. 1965;96(3):837-839. doi:10.1042/bj0960837

41. Westlake EK, Campbell EJM. Effects of aminophylline, nikethamide, and sodium salicylate in respiratory failure. BMJ. 1959;1 (5117):274-276. doi:10.1136/bmj.1.5117.274

42. Lister BJ, Poland M, DeLapp RE. Efficacy of nabumetone versus diclofenac, naproxen, ibuprofen, and piroxicam in osteoarthritis and rheumatoid arthritis. Am J Med. 1993;95(2):S2-S9. doi:10.1016/0002-9343(93)90390-B

43. Hsu KHK. Thirty years after isoniazid. JAMA. 1984;251 (10):1283. doi:10.1001/jama.1984.03340340023018

44. Andersen JB, Engeland A, Owe JF, Gilhus NE. Myasthenia gravis requiring pyridostigmine treatment in a national population cohort. Eur J Neurol. 2010;17(12):1445-1450. doi:10.1111/ j.1468-1331.2010.03089.x

45. Bostock C, McDonald C. Antimuscarinics in older people: dry mouth and beyond. Dent Update. 2016;43(2):186-191. doi:10.12968/denu.2016.43.2.186

46. Friedman H, Greenblatt DJ, Scavone JM, et al. Clearance of the antihistamine doxylamine. Clin Pharmacokinet. 1989;16(5):312316. doi:10.2165/00003088-198916050-00003

47. Walan A, Bader JP, Classen M, et al. Effect of omeprazole and ranitidine on ulcer healing and relapse rates in patients with benign gastric ulcer. $N$ Engl J Med. 1989;320(2):69-75. doi:10.1056/NEJM198901123200201

48. Wang Z, Vince R. Design and synthesis of dual inhibitors of HIV reverse transcriptase and integrase: introducing a diketoacid functionality into delavirdine. Bioorg Med Chem. 2008;16(7):35873595. doi:10.1016/j.bmc.2008.02.007

49. Te Velthuis AJW, Zubkova TG, Shaw M, et al. Enisamium reduces influenza virus shedding and improves patient recovery by inhibiting viral RNA polymerase activity. Antimicrob Agents Chemother. 2021;65(4). doi:10.1128/AAC.02605-20

50. Ahmed M, Rocha JBT, Corrêa M, et al. Inhibition of two different cholinesterases by tacrine. Chem Biol Interact. 2006;162(2):165171. doi:10.1016/j.cbi.2006.06.002
51. Toal CB, Meredith PA, Elliott HL. Long-acting dihydropyridine calcium-channel blockers and sympathetic nervous system activity in hypertension: a literature review comparing amlodipine and nifedipine GITS. Blood Press. 2012;21(Suppl 1):3-10. doi:10.31 09/08037051.2012.690615

52. Wang JG, Kario K, Lau T, et al. Use of dihydropyridine calcium channel blockers in the management of hypertension in Eastern Asians: a scientific statement from the Asian Pacific Heart Association. Hypertens Res. 2011;34(4):423-430. doi:10.1038/ hr.2010.259

53. Yet L. 1,4-dihydropyridines. In: Privileged Structures in Drug Discovery. Hoboken, NJ, USA: John Wiley \& Sons, Inc.; 2018:59-82. doi:10.1002/9781118686263.ch3

54. Vaast P, Dubreucq-Fossaert S, Houfflin-Debarge V, et al. Acute pulmonary oedema during nicardipine therapy for premature labour. Eur J Obstet Gynecol Reprod Biol. 2004;113(1):98-99. doi:10.1016/j.ejogrb.2003.05.004

55. Alousi AA, Johnson DC. Pharmacology of the bipyridines: amrinone and milrinone. Circulation. 1986;73(3 Pt 2):III10-III24.

56. Leoni A, Frosini M, Locatelli A, et al. 4-Imidazo[2,1-b]thiazole-1,4DHPs and neuroprotection: preliminary study in hits searching. Eur J Med Chem. 2019;169:89-102. doi:10.1016/j.ejmech.2019.02.075

57. de Los Ríos C, Marco-Contelles J. Tacrines for Alzheimer's disease therapy. III. The PyridoTacrines. Eur J Med Chem. 2019;166:381-389. doi:10.1016/j.ejmech.2019.02.005

58. Xu D, Sun D, Wang W, et al. Discovery of pyrrolo[2,3-d]pyrimidine derivatives as potent Axl inhibitors: design, synthesis and biological evaluation. Eur J Med Chem. 2021;220:113497. doi:10.1016/j.ejmech.2021.113497

59. Jian Y, Hulpia F, Risseeuw MDP, et al. Synthesis and structure activity relationships of cyanopyridone based anti-tuberculosis agents. Eur J Med Chem. 2020;201:112450. doi:10.1016/j. ejmech.2020.112450

60. Zhang Y, Pavlova OA, Chefer SI, et al. 5-substituted derivatives of 6-halogeno-3-((2-(S)-azetidinyl)methoxy)pyridine and 6-halogeno-3-((2-(S)-pyrrolidinyl)methoxy)pyridine with low picomolar affinity for $\alpha 4 \beta 2$ nicotinic acetylcholine receptor and wide range of lipophilicity: potential probe. J Med Chem . 2004;47(10):24532465. doi:10.1021/jm030432v

61. Yang G-Z, Shang X-F, Cheng P-L, et al. Facile three-component synthesis, insecticidal and antifungal evaluation of novel dihydropyridine derivatives. Molecules. 2018;23(10):2422. doi:10.33 90/molecules23102422

62. Abdelriheem N, Ahmad S, Abdelhamid A. Synthesis of some new Thieno[2,3-b]pyridines, Pyrimidino[4',5':4,5]thieno[2,3-b] pyridine and pyridines incorporating 5-bromobenzofuran-2-yl moiety. Molecules. 2015;20(1):822-838. doi:10.3390/molecules 20010822

63. Gilhus NE, Verschuuren JJ. Myasthenia gravis: subgroup classification and therapeutic strategies. Lancet Neurol. 2015;14 (10):1023-1036. doi:10.1016/S1474-4422(15)00145-3

64. Bugajski J, Głód R, Gadek-Michalska A, Bugajski AJ. Involvement of constitutive (COX-1) and inducible cyclooxygenase (COX-2) in the adrenergic-induced ACTH and corticosterone secretion. J Physiol Pharmacol. 2001;52(4 Pt 2):795-809.

65. Carty TJ, Marfat A, Moore PF, Falkner FC, Twomey TM, Weissman A. Ampiroxicam, an anti-inflammatory agent which is a prodrug of piroxicam. Agents Actions. 1993;39(3-4):157165. doi:10.1007/BF01998969

66. Kothekar V, Sahi S, Srinivasan M, Mohan A, Mishra J. Recognition of cyclooxygenase-2 (COX-2) active site by NSAIDs: a computer modelling study. Indian $J$ Biochem Biophys. 2001;38(1-2):56-63.

67. Esteve J, Farré AJ, Roser R. Pharmacological profile of droxicam. Gen Pharmacol. 1988;19(1):49-54. doi:10.1016/0306-3623(88) 90004-3 
68. Berg J, Fellier H, Christoph T, Grarup J, Stimmeder D. The analgesic NSAID lornoxicam inhibits cyclooxygenase (COX)$1 /-2$, inducible nitric oxide synthase (iNOS), and the formation of interleukin (IL)-6 in vitro. Inflamm Res. 1999;48(7):369-379. doi:10.1007/s000110050474

69. Finch JS, DeKornfeld TJ. Clonixin: a clinical evaluation of a new oral analgesic. J Clin Pharmacol New Drugs. 1971;11(5):371377.

70. Capone ML, Tacconelli S, Di Francesco L, Sacchetti A, Sciulli MG, Patrignani P. Pharmacodynamic of cyclooxygenase inhibitors in humans. Prostaglandins Other Lipid Mediat. 2007;82(14):85-94. doi:10.1016/j.prostaglandins.2006.05.019

71. Sigel E, Steinmann ME. Structure, function, and modulation of GABAA receptors. J Biol Chem. 2012;287(48):40224-40231. doi:10.1074/jbc.R112.386664

72. Dulfano MJ. Nikethamide as a respiratory analeptic. JAMA. 1963;185(2):69. doi:10.1001/jama.1963.03060020029016

73. Pym AS, Domenech P, Honore N, Song J, Deretic V, Cole ST. Regulation of catalase-peroxidase (KatG) expression, isoniazid sensitivity and virulence by furA of Mycobacterium tuberculosis. Mol Microbiol. 2001;40(4):879-889. doi:10.1046/j.1365-2958.20 01.02427.x

74. Armstrong S, Merrill AR. Toward the elucidation of the catalytic mechanism of the Mono-ADP-Ribosyltransferase activity of Pseudomonas aeruginosa Exotoxin A †. Biochemistry. 2004;43 (1):183-194. doi:10.1021/bi034772u

75. Vogiatzi P, Claudio PP. Efficacy of Abiraterone acetate in postdocetaxel castration-resistant prostate cancer. Expert Rev Anticancer Ther. 2010;10(7):1027-1030. doi:10.1586/era.10.84

76. Qizilbash N, Whitehead A, Higgins J, et al. Cholinesterase inhibition for Alzheimer disease. JAMA. 1998;280(20):1777. doi:10.1001/jama.280.20.1777

77. Freimuth WW. Delavirdine mesylate, a potent non-nucleoside HIV-1 reverse transcriptase inhibitor. Adv Exp Med Biol. 1996;394:279-289. doi:10.1007/978-1-4757-9209-6_25

78. Yang YX, Lewis JD, Epstein S, Metz DC. Long-term proton pump inhibitor therapy and risk of hip fracture. JAMA. 2006;296(24):2947-2953. doi:10.1001/jama.296.24.2947

79. Kukovetz WR, Holzmann S, Pöch G. Molecular mechanism of action of nicorandil. J Cardiovasc Pharmacol. 1992;20(Suppl 3): S1-S7. doi:10.1097/00005344-199206203-00002

80. Overington JP, Al-Lazikani B, Hopkins AL. How many drug targets are there? Nat Rev Drug Discov. 2006;5(12):993-996. doi:10.1038/nrd2199

81. Suter DM, Preynat-Seauve O, Tirefort D, Feki A, Krause K-H. Phenazopyridine induces and synchronizes neuronal differentiation of embryonic stem cells. J Cell Mol Med. 2009;13(9B):35173527. doi:10.1111/j.1582-4934.2009.00660.x

82. Vuori ML, Kaila T, Iisalo E, Saari KM. Systemic absorption and anticholinergic activity of topically applied tropicamide. J Ocul Pharmacol. 1994;10(2):431-437. doi:10.1089/jop.1994.10.431

83. Harvey JL, Paine AJ, Maurel P, Wright MC. Effect of the adrenal 11-beta-hydroxylase inhibitor metyrapone on human hepatic cytochrome P-450 expression: induction of cytochrome P-450 3A4. Drug Metab Dispos. 2000;28(1):96-101.

84. Little JT, Walsh S, Aisen PS. An update on huperzine A as a treatment for Alzheimer's disease. Expert Opin Investig Drugs. 2008;17(2):209-215. doi:10.1517/13543784.17.2.209

85. Nada T, Nomura M, Koshiba K, Kawano T, Mikawa J, Ito S. Clinical study with azelnidipine in patients with essential hypertension. Antiarteriosclerotic and cardiac hypertrophy-inhibitory effects and influence on autonomic nervous activity. Arzneimittelforschung. 2007;57(11):698-704. doi:10.1055/s-0031-1296670
86. Minami J, Numabe A, Andoh N, et al. Comparison of once-daily nifedipine controlled-release with twice-daily nifedipine retard in the treatment of essential hypertension. Br J Clin Pharmacol. 2004;57(5):632-639. doi:10.1111/j.1365-2125.2003.02056.x

87. Rosenthal J. Nilvadipine: profile of a new calcium antagonist. An overview. J Cardiovasc Pharmacol. 1994;24(Suppl 2):S92-S107. doi:10.1097/00005344-199412001-00014

88. Dong CJ, Guo Y, Agey P, Wheeler L, Hare WA. Nimodipine enhancement of $\alpha 2$ adrenergic modulation of NMDA receptor via a mechanism independent of $\mathrm{Ca}^{2+}$ channel blocking. Invest Ophthalmol Vis Sci. 2010;51(8):4174 4180. doi:10.1167/iovs.09-4613

89. Johnson R, Dludla P, Mabhida S, Benjeddou M, Louw J, February F. Pharmacogenomics of amlodipine and hydrochlorothiazide therapy and the quest for improved control of hypertension: a mini review. Heart Fail Rev. 2019;24(3):343-357. doi:10.1007/ s10741-018-09765-y

90. Fletcher H, Roberts G, Mullings A, Forrester T. An open trial comparing isradipine with hydralazine and methyl dopa in the treatment of patients with severe pre-eclampsia. J Obstet Gynaecol. 1999;19(3):235-238. doi:10.1080/01443619964977

91. Thayer SA, Welcome M, Chhabra A, Fairhurst AS. Effects of dihydropyridine calcium channel blocking drugs on rat brain muscarinic and alpha-adrenergic receptors. Biochem Pharmacol. 1985;34(2):175-180. doi:10.1016/0006-2952(85)90121-2

92. Malhotra HS, Plosker GL. Barnidipine. Drugs. 2001;61(7):989996. doi:10.2165/00003495-200161070-00007

93. Mielcarek J, Grobelny P, Szamburska O. The effect of betacarotene on the photostability of nisoldipine. Methods Find Exp Clin Pharmacol. 2005;27(3):167-171. doi:10.1358/mf.2005.27. 3.890873

94. Regulla S, Schneider T, Nastainczyk W, Meyer HE, Hofmann F. Identification of the site of interaction of the dihydropyridine channel blockers nitrendipine and azidopine with the calciumchannel alpha 1 subunit. EMBO J. 1991;10(1):45-49. doi:10. 1002/j.1460-2075.1991.tb07919.x

95. Zhang JG, Dehal SS, Ho T, et al. Human cytochrome p450 induction and inhibition potential of clevidipine and its primary metabolite h152/81. Drug Metab Dispos. 2006;34(5):734-737. doi:10.1124/dmd.105.006569

96. Liu Z, Zheng X, Yang X, Wang E, Wang J. Affinity and specificity of levamlodipine-human serum albumin interactions: insights into its carrier function. Biophys J. 2009;96(10):3917-3925. doi:10.1016/j.bpj.2008.12.3965

97. Tanaka H, Shigenobu K. Efonidipine hydrochloride: a dual blocker of L- and T-type ca(2+) channels. Cardiovasc Drug Rev. 2002;20(1):81-92. doi:10.1111/j.1527-3466.2002.tb00084.x

98. Niewerth M, Kunze D, Seibold M, Schaller M, Korting HC, Hube B. Ciclopirox olamine treatment affects the expression pattern of Candida albicans genes encoding virulence factors, iron metabolism proteins, and drug resistance factors. Antimicrob Agents Chemother. 2003;47(6):1805-1817. doi:10.1128/AAC.47.6.18051817.2003

99. Shipley JB, Tolman D, Hastillo A, Hess ML. Milrinone: basic and clinical pharmacology and acute and chronic management. Am J Med Sci. 1996;311(6):286-291. doi:10.1097/00000441-199606 000-00011

100. Kobayashi T, Sugawara Y, Ohkubo T, Imamura H, Makuuchi M. Effects of amrinone on hepatic ischemia-reperfusion injury in rats. J Hepatol. 2002;37(1):31-38. doi:10.1016/s0168-8278(02) 00084-3

101. López B, González A, Hermida N, Laviades C, Díez J. Myocardial fibrosis in chronic kidney disease: potential benefits of torasemide. Kidney Int Suppl. 2008;74(111):S19-S23. doi:10.1038/ki.2008.512 
102. Morgan RE, Campbell SE, Yu CY, Sponseller CA, Muster HA. Comparison of the safety, tolerability, and pharmacokinetic profile of a single oral dose of pitavastatin $4 \mathrm{mg}$ in adult subjects with severe renal impairment not on hemodialysis versus healthy adult subjects. J Cardiovasc Pharmacol. 2012;60(1):42-48. doi:10.1097/FJC.0b013e318256cdf0

103. Steed ME, Rybak MJ. Ceftaroline: a new cephalosporin with activity against resistant gram-positive pathogens. Pharmacotherapy. 2010;30(4):375-389. doi:10.1592/phco.30.4. 375

104. Roger C, Roberts JA, Muller L. Clinical pharmacokinetics and pharmacodynamics of oxazolidinones. Clin Pharmacokinet. 2018;57(5):559-575. doi:10.1007/s40262-017-0601-x

105. Richards DM, Brogden RN. Ceftazidime. A review of its antibacterial activity, pharmacokinetic properties and therapeutic use. Drugs. 1985;29(2):105-161. doi:10.2165/00003495-19852902000002

106. Markham A. Delafloxacin: first global approval. Drugs. 2017;77 (13):1481-1486. doi:10.1007/s40265-017-0790-5

107. Morlock GP, Metchock B, Sikes D, Crawford JT, Cooksey RC. ethA, inhA, and katG loci of ethionamide-resistant clinical Mycobacterium tuberculosis isolates. Antimicrob Agents Chemother. 2003;47(12):3799-3805. doi:10.1128/AAC.47.12.37 99-3805.2003

108. Ambrose Z, Herman BD, Sheen C-W, et al. The human immunodeficiency virus type 1 nonnucleoside reverse transcriptase inhibitor resistance mutation $1132 \mathrm{M}$ confers hypersensitivity to nucleoside analogs. J Virol. 2009;83(8):3826-3833. doi:10.1128/ JVI.01968-08

109. Wilby KJ, Eissa NA. Clinical pharmacokinetics and drug interactions of doravirine. Eur J Drug Metab Pharmacokinet. 2018;43 (6):637-644. doi:10.1007/s13318-018-0497-3

110. Wittayanarakul K, Hannongbua S, Feig M. Accurate prediction of protonation state as a prerequisite for reliable MM-PB(GB)SA binding free energy calculations of HIV-1 protease inhibitors. $J$ Comput Chem. 2008;29(5):673-685. doi:10.1002/jcc.20821

111. Cohen EE, Rosen LS, Vokes EE, et al. Axitinib is an active treatment for all histologic subtypes of advanced thyroid cancer: results from a phase II study. J Clin Oncol. 2008;26(29):47084713. doi:10.1200/JCO.2007.15.9566

112. Deininger MWN, Druker BJ. Specific targeted therapy of chronic myelogenous leukemia with imatinib. Pharmacol Rev. 2003;55 (3):401-423. doi:10.1124/pr.55.3.4

113. Flaherty KT. Chemotherapy and targeted therapy combinations in advanced melanoma. Clin Cancer Res. 2006;12(7):2366s-2370s. doi:10.1158/1078-0432.CCR-05-2505

114. Hotta K, Ueyama J, Tatsumi Y, et al. Lack of contribution of multidrug resistance-associated protein and organic anion-transporting polypeptide to pharmacokinetics of regorafenib, a novel multi-kinase inhibitor, in rats. Anticancer Res. 2015;35(9):46814689.

115. Giustini N, Bernthal NM, Bukata SV, Singh AS. Tenosynovial giant cell tumor: case report of a patient effectively treated with pexidartinib (PLX3397) and review of the literature. Clin Sarcoma Res. 2018;8:14. doi:10.1186/s13569-018-0101-2

116. Konstantinopoulos PA, Barry WT, Birrer M, et al. Olaparib and $\alpha$ specific PI3K inhibitor alpelisib for patients with epithelial ovarian cancer: a dose-escalation and dose-expansion phase $1 \mathrm{~b}$ trial. Lancet Oncol. 2019;20(4):570-580. doi:10.1016/S1470-2045(18) 30905-7

117. Shaw AT, Felip E, Bauer TM, et al. Lorlatinib in non-small-cell lung cancer with ALK or ROS1 rearrangement: an international, multicentre, open-label, single-arm first-in-man phase 1 trial. Lancet Oncol. 2017;18(12):1590-1599. doi:10.1016/S1470-2045 (17)30680-0
118. Wu J, Zhang M, Liu D. Acalabrutinib (ACP-196): a selective second-generation BTK inhibitor. J Hematol Oncol. 2016;9:21. doi:10.1186/s13045-016-0250-9

119. Gelbert LM, Cai S, Lin X, et al. Preclinical characterization of the CDK4/6 inhibitor LY2835219: in-vivo cell cycle-dependent/independent anti-tumor activities alone/in combination with gemcitabine. Invest New Drugs. 2014;32(5):825-837. doi:10.1007/s106 37-014-0120-7

120. Burstein HJ, Sun Y, Dirix LY, et al. Neratinib, an irreversible ErbB receptor tyrosine kinase inhibitor, in patients with advanced ErbB2-positive breast cancer. J Clin Oncol. 2010;28(8):13011307. doi:10.1200/JCO.2009.25.8707

121. Mondesir J, Willekens C, Touat M, de Botton S. IDH1 and IDH2 mutations as novel therapeutic targets: current perspectives. $J$ Blood Med. 2016;7:171-180. doi:10.2147/JBM.S70716

122. Shore SN, Britnell SR, Brown JN. Safety analysis of long-term phenazopyridine use for radiation cystitis. J Oncol Pharm Pract. 2020;26(2):306-311. doi:10.1177/1078155219842646

123. Murdin L, Hussain K, Schilder AGM. Betahistine for symptoms of vertigo. Cochrane Database Syst Rev. 2016;(6):CD010696. doi:10.1002/14651858.CD010696.pub2

124. Chen X, Ji ZL, Chen YZ. TTD: therapeutic target database. Nucleic Acids Res. 2002;30(1):412-415. doi:10.1093/nar/30.1. 412

125. Lindquist S, Stangel M. Update on treatment options for LambertEaton myasthenic syndrome: focus on use of amifampridine. Neuropsychiatr Dis Treat. 2011;7:341-349. doi:10.2147/NDT. S10464

126. Allen GS, Ahn HS, Preziosi TJ, et al. Cerebral arterial spasm-a controlled trial of nimodipine in patients with subarachnoid hemorrhage. $N$ Engl J Med. 1983;308(11):619-624. doi:10.10 56/NEJM198303173081103

127. Tagawa M, Kano M, Okamura N, et al. Neuroimaging of histamine H1-receptor occupancy in human brain by positron emission tomography (PET): a comparative study of ebastine, a secondgeneration antihistamine, and (+)-chlorpheniramine, a classical antihistamine. Br J Clin Pharmacol. 2001;52(5):501-509. doi:10. 1046/j.1365-2125.2001.01471.x

128. Webbon PM, Woolliscroft GJ. Cautious use of flunixin advocated. Vet Rec. 1984;115(2):45. doi:10.1136/vr.115.2.45-b

129. Sang Y, Barbosa JM, Wu H, Locy RD, Singh NK. Identification of a pyridoxine (pyridoxamine) 5'-phosphate oxidase from Arabidopsis thaliana. FEBS Lett. 2007;581(3):344-348. doi:10.10 16/j.febslet.2006.12.028

130. Bell J. Amlexanox for the treatment of recurrent aphthous ulcers. Clin Drug Investig. 2005;25(9):555-566. doi:10.2165/00044011200525090-00001

131. Wilhelm S, Carter C, Lynch M, et al. Discovery and development of sorafenib: a multikinase inhibitor for treating cancer. Nat Rev Drug Discov. 2006;5(10):835-844. doi:10.1038/nrd2130

132. Oliveira EF, Cerqueira NM, Fernandes PA, Ramos MJ. Mechanism of formation of the internal aldimine in pyridoxal 5phosphate-dependent enzymes. $J$ Am Chem Soc. 2011;133 (39):15496-15505. doi: $10.1021 / \mathrm{ja} 204229 \mathrm{~m}$

133. Ramadan AA, Mandil H. Spectrophotometric determination of carbinoxamine maleate in pharmaceutical formulations by ternary complex formation with $\mathrm{Cu}(\mathrm{II})$ and eosin. Anal Biochem. 2006;353(1):133-137. doi:10.1016/j.ab.2006.02.020

134. Dring AM, Anderson LE, Qamar S, Stoner MA. Rational quantitative structure-activity relationship (RQSAR) screen for PXR and CAR isoform-specific nuclear receptor ligands. Chem Biol Interact. 2010;188(3):512-525. doi:10.1016/j.cbi.2010.09.018

135. Bökesoy TA, Onaran HO. Atypical Schild plots with histamine H1 receptor agonists and antagonists in the rabbit aorta. Eur J Pharmacol. 1991;197(1):49-56. doi:10.1016/0014-2999(91)90363-u 
136. Matsumoto K, Okamoto K, Ashizawa N, Nishino T. FYX-051: a novel and potent hybrid-type inhibitor of xanthine oxidoreductase. J Pharmacol Exp Ther. 2011;336(1):95-103. doi:10.1124/ jpet.110.174540

137. Roskos KV, Bircher AJ, Maibach HI, Guy RH. Pharmacodynamic measurements of methyl nicotinate percutaneous absorption: the effect of aging on microcirculation. Br J Dermatol. 1990;122 (2):165-171. doi:10.1111/j.1365-2133.1990.tb08262.x

138. Kamanna VS, Kashyap ML. Mechanism of action of niacin. Am J Cardiol. 2008;101(8):S20-S26. doi:10.1016/j.amjcard.2008.02.029

139. Reeder NL, Kaplan J, Xu J, et al. Zinc pyrithione inhibits yeast growth through copper influx and inactivation of iron-sulfur proteins. Antimicrob Agents Chemother. 2011;55(12):5753-5760. doi:10.1128/AAC.00724-11

140. Jackson KJ, Marks MJ, Vann RE, et al. Role of $\alpha 5$ nicotinic acetylcholine receptors in pharmacological and behavioral effects of nicotine in mice. J Pharmacol Exp Ther. 2010;334(1):137-146. doi:10.1124/jpet.110.165738

141. Vormfelde SV, Sehrt D, Toliat MR, et al. Genetic variation in the renal sodium transporters $\mathrm{NKCC} 2, \mathrm{NCC}$, and $\mathrm{ENaC}$ in relation to the effects of loop diuretic drugs. Clin Pharmacol Ther. 2007;82 (3):300-309. doi:10.1038/sj.clpt.6100131

142. Wu RL, Anthes JC, Kreutner W, Harris AG, West RE Desloratadine inhibits constitutive and histamine-stimulated Nuclear Factor- $\kappa \mathrm{B}$ activity consistent with inverse agonism at the histamine H1 receptor. Int Arch Allergy Immunol. 2004;135 (4):313-318. doi:10.1159/000082325

143. Allinson RW, Gerber DS, Bieber S, Hodes BL. Reversal of mydriasis by dapiprazole. Ann Ophthalmol. 1990;22(4):131-133.

144. Tan KR, Rudolph U, Lüscher C. Hooked on benzodiazepines: GABAA receptor subtypes and addiction. Trends Neurosci. 2011;34(4):188-197. doi:10.1016/j.tins.2011.01.004

145. Ogihara T, Kano T, Kakinuma C. Evaluation of the inhibitory effect of dihydropyridines on $\mathrm{N}$-type calcium channel by virtual threedimensional pharmacophore modeling. Arzneimittelforschung. 2011;59(06):283-288. doi:10.1055/s-0031-1296398

146. Claro E, Arbonés L, García A, Picatoste F. Phosphoinositide hydrolysis mediated by histamine H1-receptors in rat brain cortex. Eur J Pharmacol. 1986;123(2):187-196. doi:10.1016/0014 2999(86)90659-X

147. Karlgren M, Vildhede A, Norinder U, et al. classification of inhibitors of hepatic organic anion transporting polypeptides (OATPs): influence of protein expression on drug-drug interactions. $J$ Med Chem. 2012;55(10):4740-4763. doi:10.1021/jm300212s

148. Broadhurst CL, Domenico P. Clinical studies on chromium picolinate supplementation in Diabetes Mellitus - a review. Diabetes Technol Ther. 2006;8(6):677-687. doi:10.1089/dia.2006.8.677

149. Hua Y, Clark S, Ren J, Sreejayan N. Molecular mechanisms of chromium in alleviating insulin resistance. $J$ Nutr Biochem. 2012;23(4):313-319. doi:10.1016/j.jnutbio.2011.11.001

150. Negro A, Martelletti P. Gepants for the treatment of migraine. Expert Opin Investig Drugs. 2019;28(6):555-567. doi:10.1080/ 13543784.2019.1618830

151. Monteseirin J, Chacon P, Vega A, et al. L-selectin expression on neutrophils from allergic patients. Clin Exp Allergy. 2005;35 (9):1204-1213. doi:10.1111/j.1365-2222.2005.02320.x

152. Jordan EJ, Kelly CM. Vemurafenib for the treatment of melanoma. Expert Opin Pharmacother. 2012;13(17):2533-2543. doi:10.1517/14656566.2012.737780

153. Dummer R, Urosevic M, Kempf W, Hoek K, Hafner J, Burg G. Imiquimod in basal cell carcinoma: how does it work? $\mathrm{Br} J$ Dermatol. 2003;149(s66):57-58. doi:10.1046/j.0366-077X.2003. 05630.x
154. Bébéar CM, Grau O, Charron A, Renaudin H, Gruson D, Bébéar C. Cloning and nucleotide sequence of the DNA gyrase (gyrA) gene from Mycoplasma hominis and characterization of quinolone-resistant mutants selected in vitro with trovafloxacin. Antimicrob Agents Chemother. 2000;44(10):2719-2727. doi:10. 1128/AAC.44.10.2719-2727.2000

155. Gootz TD, Zaniewski RP, Haskell SL, Kaczmarek FS, Maurice AE. Activities of trovafloxacin compared with those of other fluoroquinolones against purified topoisomerases and gyrA and grlA mutants of Staphylococcus aureus. Antimicrob Agents Chemother. 1999;43(8):1845-1855. doi:10.1128/AAC.43.8.1845

156. Bortolin M, Bidossi A, De Vecchi E, Avveniente M, Drago L. In vitro antimicrobial activity of chlorquinaldol against microorganisms responsible for skin and soft tissue infections: comparative evaluation with gentamicin and fusidic acid. Front Microbiol. 2017;8. doi:10.3389/fmicb.2017.01039.

157. Andersen A. Final amended report on the safety assessment of oxyquinoline and oxyquinoline sulfate as used in cosmetics1. Int $J$ Toxicol. 2006;25(1 suppl):1-9. doi:10.1080/10915810600716570

158. Merlos M, Giral M, Balsa D, et al. Rupatadine, a new potent, orally active dual antagonist of histamine and platelet-activating factor (PAF). J Pharmacol Exp Ther. 1997;280(1):114-121.

159. Asrani CH, Damle SS, Ghotge VV, et al. Efficacy and safety of metronidazole versus a combination of metronidazole and diiodohydroxyquinoline for the treatment of patients with intestinal amebiasis: a primary care physician research group study. Curr Ther Res. 1995;56(7):678-683. doi:10.1016/0011-393X(95)85137-2

160. Weber M, Breier M, Ko D, Thangaraj N, Marzan DE, Swerdlow NR. Evaluating the antipsychotic profile of the preferential PDE10A inhibitor, papaverine. Psychopharmacology. 2009;203 (4):723-735. doi:10.1007/s00213-008-1419-x

161. Belenky P, Bogan KL, Brenner C. NAD+ metabolism in health and disease. Trends Biochem Sci. 2007;32(1):12-19. doi:10.1016/ j.tibs.2006.11.006

162. Slater AFG, Cerami A. Inhibition by chloroquine of a novel haem polymerase enzyme activity in malaria trophozoites. Nature. 1992;355(6356):167-169. doi:10.1038/355167a0

163. Krueger D, Demir IE, Ceyhan GO, Zeller F, Schemann M. bis-(phydroxyphenyl)-pyridyl-2-methane (BHPM)-The active metabolite of the laxatives bisacodyl and sodium picosulfate-enhances contractility and secretion in human intestine in vitro. Neurogastroenterol Motil. 2018;30(7):e13311. doi:10.1111/ nmo. 13311

164. Wong DF, Rosenberg PB, Zhou Y, et al. In vivo imaging of amyloid deposition in Alzheimer disease using the radioligand 18 F-AV-45 (Flobetapir F 18). J Nucl Med. 2010;51(6):913-920. doi:10.2967/jnumed.109.069088

165. Fitch CD, Ferriprotoporphyrin IX. phospholipids, and the antimalarial actions of quinoline drugs. Life Sci. 2004;74(16):19571972. doi:10.1016/j.lfs.2003.10.003

166. Sheets MF, Fozzard HA, Lipkind GM, Hanck DA. Sodium channel molecular conformations and antiarrhythmic drug affinity. Trends Cardiovasc Med. 2010;20(1):16-21. doi:10.1016/j.tcm.2010.03.002

167. Mann KV, Crowe JP, Tietze KJ. Nonsedating histamine H1receptor antagonists. Clin Pharm. 1989;8(5):331-344.

168. Naline E, Trifilieff A, Fairhurst RA, Advenier C, Molimard M. Effect of indacaterol, a novel long-acting 2-agonist, on isolated human bronchi. Eur Respir J. 2007;29(3):575-581. doi:10.1183/ 09031936.00032806

169. Mahoney CE, Mochizuki T, Scammell TE. Dual orexin receptor antagonists increase sleep and cataplexy in wild type mice. Sleep. 2020;43(6). doi:10.1093/sleep/zsz302

170. Cairns P, Srivastava S, Grizzle WE. Renal cell carcinoma. Cancer Biomark. 2011;9(1-6):461-473. doi:10.3233/CBM-2011-0176 
171. Shin JJ, Saadabadi A. Trazodone; 2021.

172. Simons FE, Simons KJ. Histamine and H1-antihistamines: celebrating a century of progress. J Allergy Clin Immunol. 2011;128 (6):1139-1150.e4. doi:10.1016/j.jaci.2011.09.005

173. Andrews CD, Heneine W. Cabotegravir long-acting for HIV-1 prevention. Curr Opin HIV AIDS. 2015;10(4):258-263. doi:10.1097/COH.0000000000000161

174. Farrell DJ, Shackcloth J, Barbadora KA, Green MD. Streptococcus pyogenes isolates with high-level macrolide resistance and reduced susceptibility to telithromycin associated with 23S rRNA mutations. Antimicrob Agents Chemother. 2006;50 (2):817-818. doi:10.1128/AAC.50.2.817-818.2006

175. Dündar Y, Dodd S, Strobl J, Boland A, Dickson R, Walley T. Comparative efficacy of newer hypnotic drugs for the short-term management of insomnia: a systematic review and meta-analysis. Hum Psychopharmacol Clin Exp. 2004;19(5):305-322. doi:10.1002/ hup. 594

176. Rucins M, Kaldre D, Pajuste K, et al. Synthesis and studies of calcium channel blocking and antioxidant activities of novel 4pyridinium and/or N-propargyl substituted 1,4-dihydropyridine derivatives. Comptes Rendus Chim. 2014;17(1):69-80. doi:10. 1016/j.crci.2013.07.003

177. Peri R, Padmanabhan S, Rutledge A, Singh S, Triggle DJ. Permanently charged chiral 1,4-dihydropyridines: molecular probes of L-type calcium channels. Synthesis and pharmacological characterization of methyl ( $\omega$-trimethylalkylammonium) 1,4-dihydro-2,6dimethyl-4-(3- nitrophenyl)-3,5-pyridinedicarboxylate iodide. $J$ Med Chem. 2000;43(15):2906-2914. doi:10.1021/jm0000281

178. Baindur N, Rutledge A, Triggle DJ. A homologous series of permanently charged 1,4-dihydropyridines: novel probes designed to localize drug binding sites on ion channels. J Med Chem. 1993;36(23):3743-3745. doi:10.1021/jm00075a 034

179. Fu Q, Sanbe H, Kagawa C, Kunimoto KK, Haginaka J. Uniformly sized molecularly imprinted polymer for (S)-nilvadipine. Comparison of chiral recognition ability with HPLC chiral stationary phases based on a protein. Anal Chem. 2003;75 (2):191-198. doi:10.1021/ac026039z

180. Zhou K, Wang XM, Zhao YZ, Cao YX, Fu Q, Zhang SQ. Synthesis and antihypertensive activity evaluation in spontaneously hypertensive rats of nitrendipine analogues. Med Chem Res. 2011;20(8):1325-1330. doi:10.1007/s00044-010-9477-0

181. Zarghi A, Sadeghi H, Fassihi A, Faizi M, Shafiee A. Synthesis and calcium antagonist activity of 1,4-dihydropyridines containing phenylaminoimidazolyl substituents. Farmaco. 2003;58 (11):1077-1081. doi:10.1016/S0014-827X(03)00159-9

182. Kumar RS, Idhayadhulla A, Abdul Nasser AJ, Selvin J. Synthesis and anticoagulant activity of a new series of 1,4-dihydropyridine derivatives. Eur J Med Chem. 2011;46(2):804-810. doi:10.1016/j. ejmech.2010.12.006

183. Mooradian AD. Dyslipidemia in type 2 diabetes mellitus. Nat Rev Endocrinol. 2009;5(3):150-159. doi:10.1038/ncpendmet1066

184. Abu Farha R, Bustanji Y, Al-Hiari Y, Al-Qirim T, Abu Shiekha G, Albashiti R. Lipid lowering activity of novel N -(benzoylphenyl) pyridine-3-carboxamide derivatives in Triton WR-1339-induced hyperlipidemic rats. $J$ Enzyme Inhib Med Chem. 2016;31 (sup4):138-144. doi:10.1080/14756366.2016.1222581

185. Kumar A, Maurya RA, Sharma S, Kumar M, Bhatia G. Synthesis and biological evaluation of $\mathrm{N}$-aryl-1,4-dihydropyridines as novel antidyslipidemic and antioxidant agents. Eur $J$ Med Chem. 2010;45(2):501-509. doi:10.1016/j.ejmech.2009.10.036

186. Jo YW, Im WB, Rhee JK, Shim MJ, Kim WB, Choi EC. Synthesis and antibacterial activity of oxazolidinones containing pyridine substituted with heteroaromatic ring. Bioorganic Med Chem. 2004;12(22):5909-5915. doi:10.1016/j.bmc.2004.08.025
187. Reen GK, Kumar A, Sharma P. In vitro and in silico evaluation of 2-(substituted phenyl) oxazolo[4,5-b]pyridine derivatives as potential antibacterial agents. Med Chem Res. 2017;26 (12):3336-3344. doi:10.1007/s00044-017-2026-3

188. Salem MS, Ali MAM. Novel pyrazolo[3,4-b]pyridine derivatives: synthesis, characterization, antimicrobial and antiproliferative profile. Biol Pharm Bull. 2016;39(4):473-483. doi:10.1248/bpb. b15-00586

189. Khidre RE, Radini IAM. Design, synthesis and docking studies of novel thiazole derivatives incorporating pyridine moiety and assessment as antimicrobial agents. Sci Rep. 2021;11(1). doi:10.1038/s41598-021-86424-7

190. Lak SS, Souldozi A, Talebi R. Synthesis and evaluation of antibacterial activity of 1,3,4-oxadiazoles derivatives containing pyridine ring. J Chem Pharm Res. 2017;9(2):141-146.

191. Prachayasittikul S, Treeratanapiboon L, Ruchirawat S, Prachayasittikul V. Novel activities of 1-adamantylthiopyridines as antibacterials, antimalarials and anticancers. EXCLI J. 2009;8. doi:10.17877/DE290R-641

192. Sun N-B, Fu J-Q, Weng J-Q, Jin J-Z, Tan C-X, Liu X-H. Microwave assisted synthesis, antifungal activity and DFT theoretical study of some novel 1,2,4-triazole derivatives containing the 1,2,3-thiadiazole moiety. Molecules. 2013;18(10):1272512739. doi:10.3390/molecules 181012725

193. Mu JX, Shi YX, Wu HK, et al. Microwave assisted synthesis, antifungal activity, DFT and SAR study of 1,2,4-triazolo[4,3-a] pyridine derivatives containing hydrazone moieties. Chem Cent $J$. 2016;10(1):50. doi:10.1186/s13065-016-0196-6

194. Wei LJ, Tan WQ, Zhang JJ, et al. Synthesis, characterization, and antifungal activity of Schiff bases of inulin bearing pyridine ring. Polymers (Basel). 2019;11(2):371. doi:10.3390/polym11020371

195. Jia RX, Duan YF, Fang Q, Wang XY, Huang JY. Pyridine-grafted chitosan derivative as an antifungal agent. Food Chem. 2016;196:381-387. doi:10.1016/j.foodchem.2015.09.053

196. Tan W, Li Q, Gao Z, Qiu S, Dong F, Guo Z. Design, synthesis of novel starch derivative bearing 1,2,3-triazolium and pyridinium and evaluation of its antifungal activity. Carbohydr Polym. 2017;157:236-243. doi:10.1016/j.carbpol.2016.09.093

197. Elshemy HAH, Zaki MA, Mohamed EI, Khan SI, Lamie PF. A multicomponent reaction to design antimalarial pyridyl-indole derivatives: synthesis, biological activities and molecular docking. Bioorg Chem. 2020;97:103673. doi:10.1016/j.bioorg.20 20.103673

198. Xue J, Diao JS, Cai GB, et al. Antimalarial and structural studies of pyridine-containing inhibitors of 1-deoxyxylulose-5-phosphate reductoisomerase. ACS Med Chem Lett. 2013;4(2):278-282. doi: $10.1021 / \mathrm{ml} 300419 \mathrm{r}$

199. Salem MS, Sakr SI, El-Senousy WM, Madkour HMF. Synthesis, antibacterial, and antiviral evaluation of new heterocycles containing the pyridine moiety. Arch Pharm. 2013;346(10):766-773. doi:10.1002/ardp.201300183

200. El-Sayed WA, Khalaf HS, Mohamed SF, Hussien HA, Kutkat $\mathrm{OM}$, Amr AE. Synthesis and antiviral activity of 1,2,3-triazole glycosides based substituted pyridine via click cycloaddition. Russ J Gen Chem. 2017;87(10):2444-2453. doi:10.1134/S107 0363217100279

201. Asquith CR, Berger BT, Wan J, et al. SGC-GAK-1: a chemical probe for cyclin $\mathrm{G}$ associated kinase (GAK). J Med Chem. 2019;62(5):2830-2836. doi:10.1021/acs.jmedchem.8 b01213

202. Kovackova S, Chang L, Bekerman E, et al. Selective inhibitors of cyclin $\mathrm{G}$ associated kinase (GAK) as anti-hepatitis $\mathrm{C}$ agents. $J$ Med Chem. 2015;58(8):3393-3410. doi:10.1021/jm501759m 
203. Pu SY, Wouters R, Schor S, et al. Optimization of isothiazolo[4,3b]pyridine-based inhibitors of cyclin $\mathrm{G}$ associated kinase (GAK) with broad-spectrum antiviral activity. J Med Chem. 2018;61 (14):6178-6192. doi:10.1021/acs.jmedchem.8b00613

204. Li J, Kovackova S, Pu S, et al. Isothiazolo[4,3-b]pyridines as inhibitors of cyclin $\mathrm{G}$ associated kinase: synthesis, structureactivity relationship studies and antiviral activity. Medchemcomm. 2015;6(9):1666-1672. doi:10.1039/C5MD00229J

205. Wouters R, Pu SY, Froeyen M, et al. Cyclin G-associated kinase (GAK) affinity and antiviral activity studies of a series of 3-Csubstituted isothiazolo[4,3-b]pyridines. Eur $J$ Med Chem. 2019;163:256-265. doi:10.1016/j.ejmech.2018.11.065

206. Martinez-Gualda B, Pu SY, Froeyen M, Herdewijn P, Einav S, De Jonghe S. Structure-activity relationship study of the pyridine moiety of isothiazolo[4,3-b]pyridines as antiviral agents targeting cyclin G-associated kinase. Bioorg Med Chem. 2020;28 (1):115188. doi:10.1016/j.bmc.2019.115188

207. Xu S, Rouzer CA, Marnett LJ. Oxicams, a class of nonsteroidal anti-inflammatory drugs and beyond. IUBMB Life. 2014;66 (12):803-811. doi:10.1002/iub.1334

208. Lombardino JG, Lowe JA. The role of the medicinal chemist in drug discovery - then and now. Nat Rev Drug Discov. 2004;3 (10):853-862. doi:10.1038/nrd1523

209. Croom KF, Siddiqui MAA. Etoricoxib: a review of its use in the symptomatic treatment of osteoarthritis, rheumatoid arthritis, ankylosing spondylitis and acute gouty arthritis. Drugs. 2009;69 (11):1513-1532. doi:10.2165/00003495-200969110-00008

210. Khan FA, Nasim N, Wang Y, et al. Amphiphilic desmuramyl peptides for the rational design of new vaccine adjuvants: synthesis, in vitro modulation of inflammatory response and molecular docking studies. Eur J Med Chem. 2021;209:112863. doi:10. 1016/j.ejmech.2020.112863

211. Khan FA, Ulanova M, Bai B, Yalamati D, Jiang ZH. Design, synthesis and immunological evaluation of novel amphiphilic desmuramyl peptides. Eur J Med Chem. 2017;141:26-36. doi:10.1016/j.ejmech.2017.09.070

212. Thirumurugan P, Mahalaxmi S, Perumal PT. Synthesis and antiinflammatory activity of 3-indolyl pyridine derivatives through one-pot multi component reaction. J Chem Sci. 2010;122(6):819832. doi:10.1007/s12039-010-0070-3

213. Liu H, Li Y, Wang XY, et al. Synthesis, preliminary structureactivity relationships, and in vitro biological evaluation of 6-aryl3-amino-thieno[2,3-b]pyridine derivatives as potential antiinflammatory agents. Bioorganic Med Chem Lett. 2013;23 (8):2349-2352. doi:10.1016/j.bmcl.2013.02.059

214. Yaqoob S, Nasim N, Khanam R, et al. Synthesis of highly potent anti-inflammatory compounds (ROS inhibitors) from isonicotinic acid. Molecules. 2021;26(5):1272. doi:10.3390/molecules2605 1272

215. Kuo T, McQueen A, Chen TC, Wang JC. Regulation of glucose homeostasis by glucocorticoids. Glucocorticoid Signal. 2015;99126. DOI:10.1007/978-1-4939-2895-8_5

216. Terao M, Itoi S, Matsumura S, Yang L, Murota H, Katayama I. Local glucocorticoid activation by $11 \beta$-hydroxysteroid dehydrogenase 1 in keratinocytes: the role in hapten-induced dermatitis. Am J Pathol. 2016;186(6):1499-1510. doi:10.1016/j.ajpath.20 16.01.014

217. Praveenkumar E, Gurrapu N, Kumar Kolluri P, Yerragunta V, Reddy Kunduru B, Subhashini NJP. Synthesis, anti-diabetic evaluation and molecular docking studies of 4-(1-aryl-1H-1, 2, 3triazol-4-yl)-1,4-dihydropyridine derivatives as novel 11- $\beta$ hydroxysteroid dehydrogenase-1 (11 $\beta$-HSD1) inhibitors. Bioorg Chem. 2019;90:103056. doi:10.1016/j.bioorg.2019.103056
218. Adib M, Peytam F, Rahmanian-Jazi M, et al. Design, synthesis and in vitro $\alpha$-glucosidase inhibition of novel coumarin-pyridines as potent antidiabetic agents. New J Chem. 2018;42(21):1726817278. doi:10.1039/C8NJ02495B

219. Hart RG, Pearce LA, Ravina BM, Yaltho TC, Marler JR. Neuroprotection trials in Parkinson's disease: systematic review. Mov Disord. 2009;24(5):647-654. doi:10.1002/mds.22432

220. Li B, Yang Y, Wang Y, et al. Acetylation of NDUFV1 induced by a newly synthesized HDAC6 inhibitor HGC rescues dopaminergic neuron loss in Parkinson models. iScience. 2021;24(4):10 2302. doi:10.1016/j.isci.2021.102302

221. Stephenson J, Nutma E, van der Valk P, Amor S. Inflammation in CNS neurodegenerative diseases. Immunology. 2018;154(2):204219. doi:10.1111/imm.12922

222. Norat P, Soldozy S, Sokolowski JD, et al. Mitochondrial dysfunction in neurological disorders: exploring mitochondrial transplantation. NPJ Regen Med. 2020;5(1):22. doi:10.1038/s41536-02000107-X

223. Durães F, Pinto M, Sousa E. Old drugs as new treatments for neurodegenerative diseases. Pharmaceuticals. 2018;11(2):44. doi:10.3390/ph11020044

224. Birks J, López-Arrieta J. Nimodipine for primary degenerative, mixed and vascular dementia. Cochrane Database Syst Rev. 2002. doi:10.1002/14651858.CD000147

225. Carlson AP, Hänggi D, Macdonald RL, Shuttleworth CW. Nimodipine reappraised: an old drug with a future. Curr Neuropharmacol. 2020;18(1):65-82. doi:10.2174/1570159X176 66190927113021

226. Ul Mohsin NA, Ahmad M. Donepezil: a review of the recent structural modifications and their impact on anti-Alzheimer activity. Braz J Pharm Sci. 2020;56. doi:10.1590/s2175-97902019 000418325.

227. Bai DL, Tang XC, He XC, Huperzine A. A potential therapeutic agent for treatment of Alzheimer's disease. Curr Med Chem. 2000;7(3):355-374. doi:10.2174/0929867003375281

228. Chiacchio MA, Iannazzo D, Romeo R, Giofrè SV, Legnani L. Pyridine and pyrimidine derivatives as privileged scaffolds in biologically active agents. Curr Med Chem. 2020;26(40):71667195. doi:10.2174/0929867325666180904125400

229. Abdel-Latif NA, Sabry NM, Mohamed AM, Abdulla MM. Synthesis, analgesic, and antiparkinsonian profiles of some pyridine, pyrazoline, and thiopyrimidine derivatives. Monatshefte für Chemie. 2007;138(7):715-724. doi:10.1007/s00706-007-0656-8

230. Klusa V, Klimaviciusa L, Duburs G, Poikans J, Zharkovsky A. Anti-neurotoxic effects of tauropyrone, a taurine analogue. In: Taurine 6. Springer US; 2006:499-508. doi:10.1007/978-0-38733504-9_56

231. Klimaviciusa L, Klusa V, Duburs G, Kaasik A, Kalda A, Zharkovsky A. Distinct effects of atypical 1,4-dihydropyridines on 1-methyl-4-phenylpyridinium-induced toxicity. Cell Biochem Funct. 2007;25(1):15-21. doi:10.1002/cbf.1340

232. Fernandes M. Effects of 1,4-dihydropyridine derivatives (cerebrocrast, gammapyrone, glutapyrone, and diethone) on mitochondrial bioenergetics and oxidative stress: a comparative study. Mitochondrion. 2003;3(1):47-59. doi:10.1016/S1567-7249(03) 00060-6

233. Pupure J, Isajevs S, Gordjushina V, et al. Distinct Influence of atypical 1,4-dihydropyridine compounds in azidothymidineinduced neuro- and cardiotoxicity in mice ex vivo. Basic Clin Pharmacol Toxicol. 2008;103(5):401-406. doi:10.1111/j.17427843.2008.00221.X

234. Lan YL, Fang DY, Zhao J, Ma TH, Li S. A research update on the potential roles of aquaporin 4 in neuroinflammation. Acta Neurol Belg. 2016;116(2):127-134. doi:10.1007/s13760-015-0520-2 
235. Marcinkowska M, Kołaczkowski M, Kamiński K, et al. 3-aminomethyl derivatives of 2-phenylimidazo[1,2- a]-pyridine as positive allosteric modulators of GABA A receptor with potential antipsychotic activity. ACS Chem Neurosci. 2017;8(6):12911298. doi:10.1021/acschemneuro.6b00432

236. Francisco W, Pivatto M, Danuello A, et al. Pyridine alkaloids from senna multijuga as acetylcholinesterase inhibitors. $J$ Nat Prod. 2012;75(3):408-413. doi:10.1021/np200814j

237. Serrano MAR, Pivatto M, Francisco W, et al. Acetylcholinesterase inhibitory pyridine alkaloids of the leaves of Senna multijuga. $J$ Nat Prod. 2010;73(3):482-484. doi:10.1021/np900644x

238. Ling Y, Gao W-J, Ling $\mathrm{C}$, et al. $\beta$-Carboline and N-hydroxycinnamamide hybrids as anticancer agents for drugresistant hepatocellular carcinoma. Eur $J$ Med Chem. 2019;168:515-526. doi:10.1016/j.ejmech.2019.02.054

239. Dai H, Huang M, Qian J, et al. Excellent antitumor and antimetastatic activities based on novel coumarin/pyrazole oxime hybrids. Eur J Med Chem. 2019;166:470-479. doi:10.1016/j.ejmech. 2019.01.070

240. Alqahtani AM, Bayazeed AA. Synthesis and antiproliferative activity studies of new functionalized pyridine linked thiazole derivatives. Arab J Chem. 2021;14(1):102914. doi:10.1016/j. arabjc.2020.11.020

241. Brown DG, Wobst HJ. A decade of FDA-approved drugs (20102019): trends and future directions. J Med Chem. 2021;64 (5):2312-2338. doi:10.1021/acs.jmedchem.0c01516

242. Sailaja E, Bhavani S, Rambabu D, Basaveswara rao MV, Pal M. A greener approach toward $\mathrm{N}-1$ heteroarylation of indoles: synthesis and in vitro evaluation of potential anti-proliferative agents. Arab J Chem. 2019;12(8):3667-3677. doi:10.1016/j. arabjc.2015.11.008

243. Viradiya D, Mirza S, Shaikh F, et al. Design and synthesis of 1,4dihydropyridine derivatives as anti-cancer agent. Anticancer Agents Med Chem. 2017;17(7). doi:10.2174/187152061666616 1206143251

244. Mahmoud NFH, El-Sewedy A. Facile synthesis of novel heterocyclic compounds based on pyridine moiety with pharmaceutical activities. J Heterocycl Chem. 2019;(9):1-14. doi:10.1002/ jhet.3881

245. Hamza EK, Hamdy NA, Zarie ES, Fakhr IMI, Elwahy AHM, Awad HM. Synthesis and in vitro anticancer evaluation of novel pyridine derivatives bearing tetrahydronaphthalene scaffold. Arkivoc. 2019;2019(6):459-480. doi:10.24820/ark.5550190. p011.056
246. Davari AS, Abnous K, Mehri S, Ghandadi M, Hadizadeh F. Synthesis and biological evaluation of novel pyridine derivatives as potential anticancer agents and phosphodiesterase-3 inhibitors. Bioorg Chem. 2014;57:83-89. doi:10.1016/j.bioorg.2014.09.003

247. Liu XH, Liu HF, Shen X, et al. Synthesis and molecular docking studies of novel 2-chloro-pyridine derivatives containing flavone moieties as potential antitumor agents. Bioorganic Med Chem Lett. 2010;20(14):4163-4167. doi:10.1016/j.bmcl.2010.05.080

248. Bassyouni FA, Tawfik HA, Soliman AM, Rehim MA. Synthesis and anticancer activity of some new pyridine derivatives. Res Chem Intermed. 2012;38(7):1291-1310. doi:10.1007/s11164-011-0413-9

249. Abdelazem AZ, Al-Sanea MM, Park H-M, Lee SH. Synthesis of new diarylamides with pyrimidinyl pyridine scaffold and evaluation of their anti-proliferative effect on cancer cell lines. Bioorg Med Chem Lett. 2016;26(4):1301-1304. doi:10.1016/j.bmcl.2016.01.014

250. Süss-Fink G, Khan F-A, Juillerat-Jeanneret L, Dyson PJ, Renfrew AK. Synthesis and anticancer activity of long-chain isonicotinic ester ligand-containing arene ruthenium complexes and nanoparticles. $J$ Clust Sci. 2010;21(3):313-324. doi:10.1007/s10876-010-0298-6

251. Khan FA, Therrien B, Süss-Fink G, Zava O, Dyson PJ. Arene ruthenium dichloro complexes containing isonicotinic ester ligands: synthesis, molecular structure and cytotoxicity. J Organomet Chem. 2013;730:49-56. doi:10.1016/j.jorganchem.2012.10.016

252. Eldehna WM, Altoukhy A, Mahrous H, Abdel-Aziz HA. Design, synthesis and QSAR study of certain isatin-pyridine hybrids as potential anti-proliferative agents. Eur J Med Chem. 2015;90:684-694. doi:10.1016/j.ejmech.2014.12.010

253. Chavva K, Pillalamarri S, Banda V, et al. Synthesis and biological evaluation of novel alkyl amide functionalized trifluoromethyl substituted pyrazolo[3,4-b]pyridine derivatives as potential anticancer agents. Bioorg Med Chem Lett. 2013;23(21):5893-5895. doi:10.1016/j.bmcl.2013.08.089

254. Abdel-Megeed MF, Badr BE, Azaam MM, El-Hiti GA. Synthesis, antimicrobial and anticancer activities of a novel series of diphenyl 1-(pyridin-3-yl)ethylphosphonates. Bioorg Med Chem. 2012;20(7):2252-2258. doi:10.1016/j.bmc.2012.02.015

\section{Publish your work in this journal}

Drug Design, Development and Therapy is an international, peerreviewed open-access journal that spans the spectrum of drug design and development through to clinical applications. Clinical outcomes, patient safety, and programs for the development and effective, safe, and sustained use of medicines are a feature of the journal, which has also been accepted for indexing on PubMed Central. The manuscript management system is completely online and includes a very quick and fair peer-review system, which is all easy to use. Visit http://www. dovepress.com/testimonials.php to read real quotes from published authors. 